\title{
Seismic detection of the martian core
}

\section{Journal Article}

\section{Author(s):}

Stähler, Simon Christian (D); Khan, Amir (D); Banerdt, W. Bruce; Lognonne, Philippe; Giardini, Domenico; Ceylan, Savas (D; Drilleau, Mélanie; Duran, Andrea; Garcia, Raphaël F.; Huang, Quancheng; Kim, Doyeon; Lekic, Vedran; Samuel, Henri; Schimmel, Martin; Schmerr, Nicholas; Sollberger, David (iD; Stutzmann, Éléonore; Xu, Zongbo; Antonangeli, Daniele; Charalambous,

Constantinos; Schmelzbach, Cédric (D); Zenhäusern, Géraldine (D); Clinton, John Francis (D); Dahmen, Nikolaj Louis (D); van Driel, Martin; et al.

\section{Publication date:}

2021-07-23

\section{Permanent link:}

https://doi.org/10.3929/ethz-b-000498074

\section{Rights / license:}

In Copyright - Non-Commercial Use Permitted

\section{Originally published in:}

Science 373(6553), https://doi.org/10.1126/science.abi7730 


\section{Title: Seismic detection of the Martian core}

Authors: Simon C. Stähler*1, Amir Khan ${ }^{1,2}$, W. Bruce Banerdt ${ }^{3}$, Philippe Lognonné4, Domenico Giardini $^{1}$, Savas Ceylan ${ }^{1}$, Mélanie Drilleau ${ }^{5}$, A. Cecilia Duran ${ }^{1}$, Raphaël F. Garcia ${ }^{5}$, Quancheng Huang $^{6}$, Doyeon Kim ${ }^{6}$, Vedran Lekic ${ }^{6}$, Henri Samuel $^{4}$, Martin Schimmel ${ }^{7}$, Nicholas Schmerr ${ }^{6}$,

David Sollberger ${ }^{1}$, Éléonore Stutzmann ${ }^{4}$, Zongbo $\mathrm{Xu}^{4}$, Daniele Antonangeli ${ }^{8}$, Constantinos

Charalambous ${ }^{9}$, Paul Davis ${ }^{10}$, Jessica C.E. Irving ${ }^{11}$, Taichi Kawamura ${ }^{4}$, Martin Knapmeyer ${ }^{12}$, Ross Maguire $^{6}$, Angela G. Marusiak ${ }^{3}$, Mark P. Panning ${ }^{3}$, Clément Perrin ${ }^{13}$, Ana-Catalina Plesa ${ }^{12}$, Attilio Rivoldini ${ }^{14}$, Cédric Schmelzbach ${ }^{1}$, Géraldine Zenhäusern ${ }^{1}$, Éric Beucler ${ }^{13}$, John Clinton ${ }^{15}$, Nikolaj Dahmen ${ }^{1}$, Martin van Driel ${ }^{1}$, Tamara Gudkova ${ }^{16}$, Anna Horleston ${ }^{11}$, W. Thomas Pike ${ }^{9}$, Matthieu Plasman ${ }^{4}$, Suzanne E. Smrekar ${ }^{3}$

\section{Affiliations:}

${ }^{1}$ Institute of Geophysics, ETH Zürich; Zürich, Switzerland

${ }^{2}$ Physik-Institut, University of Zürich; Zürich, Switzerland

${ }^{3}$ Jet Propulsion Laboratory, California Institute of Technology; Pasadena CA, USA

${ }^{4}$ Université de Paris, Institut de physique du globe de Paris, CNRS, 75005 Paris

${ }^{5}$ Institut Supérieur de l'Aéronautique et de l'Espace SUPAERO; Toulouse, France

${ }^{6}$ Department of Geology, University of Maryland; College Park, MD 20742 USA

${ }^{7}$ Geosciences Barcelona - CSIC; Barcelona, Spain

${ }^{8}$ Sorbonne Université, Muséum National d'Histoire Naturelle, UMR CNRS 7590, Institut de Minéralogie, de Physique des Matériaux et de Cosmochimie, IMPMC; Paris, France Kingdom

${ }^{9}$ Department of Electrical and Electronic Engineering, Imperial College; London, United

${ }^{10}$ Department of Earth, Planetary, and Space Sciences, University of California Los Angeles; Los Angeles, USA

${ }^{11}$ School of Earth Sciences, University of Bristol; Bristol, United Kingdom

${ }^{12}$ DLR Institute of Planetary Research; Berlin, Germany

${ }^{13}$ Laboratoire de Planétologie et Géodynamique, Univ. Nantes; Nantes, France

${ }^{14}$ Royal Observatory of Belgium; Brussels, Belgium

${ }^{15}$ Swiss Seismological Service (SED), ETH Zürich; Zürich, Switzerland

${ }^{16}$ Schmidt Institute of Physics of the Earth RAS; Moscow, Russia

*Corresponding author. Email: simon.staehler@erdw.ethz.ch

Abstract: Clues to a planet's geologic history are contained in its interior structure, particularly its core. We detected reflections of seismic waves from the core-mantle boundary of Mars using InSight seismic data and invert these together with geodetic data to constrain the radius of the liquid metal core to $1830 \pm 40 \mathrm{~km}$. The large core implies a Martian mantle mineralogically 
similar to the terrestrial upper mantle and transition zone, but differs from the Earth by not having a bridgmanite-dominated lower mantle. We inferred a mean core density of $5.7 \mathrm{~g} / \mathrm{cm}^{3}$ to $6.3 \mathrm{~g} / \mathrm{cm}^{3}$, requiring a substantial complement of light elements dissolved in the iron-nickel core. The seismic core shadow covers half the surface of Mars, including the majority of potentially active regions, e.g., Tharsis, possibly limiting the number of detectable marsquakes.

One-Sentence Summary: Marsquake observations confirm a large, liquid, and light Martian metal core. 


\section{Main Text:}

The core of a planet plays a prominent role because it governs many of the fundamental processes from dynamo action and magnetic field generation to mantle convection that impact the surface through volcanic and tectonic activity, and may influence the early climate through magnetic shielding of the atmosphere. The size of the Martian core is of particular interest because of its dramatic impact on the planet's evolution, which differs from the Earth's primarily as a result of Mars' smaller size, and therefore, accelerated differentiation and core formation and cooling-off that resulted a rigid-shell one-plate planet $(1,2)$. A small core with a radius between 1300-1600 km would be deficient in light elements and accommodate a Martian lower mantle similar to the Earth's, dominated by bridgmanite-structure silicates (3); a large core with a radius range between 1800-1900 km would instead be enriched in light elements, exclude the presence of lower mantle layer (4), and thereby exert a markedly different dynamic control over the Martian mantle (5-8) with implications for an early Martian dynamo (1) that could explain the observed highly magnetized crust in the southern highlands (9). Direct constraints on the core and deep interior of Mars, however, are scarce and limited to global geophysical measurements, including mass, moment of inertia, and tidal response $(10,11)$, in addition to geochemical data based on achondritic basaltic meteorites that originated from the surface of Mars (12-14). Collectively, these observations suggest a liquid core with a radius in the range $\sim 1700-1900 \mathrm{~km}$ $(4,8,11,15,16)$. However, without additional observations the range of potential Martian core sizes encompasses a large enough range to allow for either the presence or the absence of a phase transition equivalent of the "660-km" discontinuity that marks the onset of the Earth's lower mantle.

The estimate of core size can be improved with the direct detection of core-interacting seismic phases, which constrain the core size of the Earth and Moon. The Earth's core had been predicted in the late 18th century, based on the recognition that the density of near-surface rocks is substantially lower than the average density of the Earth (17), but was not confirmed until global observations of earthquakes became possible (18). Subsequent seismic measurements led to the unexpected discoveries in Earth's deep interior, such as the inner core (19) and deep mantle layering $(20,21)$. Similarly, the Apollo lunar seismic data (22) were used to establish the existence of a lunar core, including an inner core $(23,24)$. The detection of seismic waves reflected from the core therefore not only stands to refine the insights gained from studying the chemistry of the Martian rocks, whose siderophile element depletion and isotopic signature point to a core-forming event early in Mars' history $(25,26)$, but to considerably improve our understanding of the deep interior of Mars.

After a full Martian year, the Interior Exploration using Seismic Investigations, Geodesy and Heat Transport (InSight) mission (27) and its seismometer SEIS (28) has recorded a multitude of seismic events, which have been located and classified by the Marsquake Service (29-31). Of these, the low-frequency events with main energy below $1 \mathrm{~Hz}$ and waves travelling through the mantle, can be used to characterize the crustal and mantle structure of Mars $(32,33)$.

To investigate the core of Mars, we analyzed seismic data from 11 low-frequency marsquakes. Six of them were found in a suitable distance range $\left(27^{\circ}-38.5^{\circ}(30)\right)$, with high enough signal-tonoise ratio (SNR) to identify potential core-reflected $\mathrm{S}$-waves $(\mathrm{ScS}$, see Table 1). Because Swaves cannot propagate in a fluid medium, the core-mantle-boundary (CMB) acts as a polarization filter, reflecting horizontally polarized S-waves (SH waves) back into the mantle, while vertically polarized S-waves (SV) lose some energy due to S-to-P conversion. S-waves 
reflected from the CMB are therefore expected to be predominantly horizontally polarized at the receiver, with an azimuth orthogonal to the source direction. The events for which the direction could be determined from P- and S-wave polarization (S0173a and S0235b) appear to originate in the Cerberus Fossae graben system $(29,34)$, with backazimuths of $70^{\circ}-90^{\circ}$. The focal mechanisms inferred for these events confirmed normal faulting (35), resulting in relatively strong radiation of $\mathrm{SH}$ waves towards the core.

All marsquakes show an S-wave coda dominated by receiver-side scattering (Fig. 1A) (31). Within this coda, the marsquake with the highest SNR observed so far (S0173a) shows a peak around $510 \mathrm{~s}$ and $350 \mathrm{~s}$ after the main $\mathrm{P}$ - and S-wave arrivals, respectively. We used polarization filtering (36) to enhance signals that are linearly and horizontally polarized, as expected for ScS waves (Fig. 1A). After filtering, we observed matching signals for five other events (S0235b, S0407a, S0409d, S0484a, and S0325a) at a similar distance (Fig. 1B). To identify energy pulses that are consistent with $\mathrm{ScS}$, we performed a slant stack (37) for the events, using predicted travel times in 5000 mantle models compatible with surface reflected seismic body waves (32). The stack of energy in a $10 \mathrm{~s}$ time window (filtered between $0.3-0.8 \mathrm{~Hz}$ ) around the predicted $\mathrm{ScS}$ arrival time shows the presence of a signal (Fig. 1C), where a reflection from a $\sim 1600-\mathrm{km}$ depth interface is expected. This result is confirmed by a model-agnostic vespagram-stack showing a low-slowness, steep incidence-angle arrival compatible with a deep reflector (38). To refine the arrival time estimate, the time window identified in the stacking was further examined using two independent methods: (i) Manual picking in polarization-filtered filter banks to recognize potential artefacts from instrument or wind and (ii) Coda correlation, using the Swaveform as matching filter, assuming that the $\mathrm{S}$-wave train has high similarity to $\mathrm{ScS}(38)$. This resulted in a set of picks for each event (Fig 1D). The results from the two methods were compared to the event slant-stacks to remove misidentified signals and consolidated into a single set of picks (Table 1).

While the SNR of the ScS phases is around 2 or less, the signal is significant compared to the energy of the S-wave coda, wind noise, and known instrument artefacts $(38,39)$. From travel time tables computed in seismic velocity models compatible with observations so far $(8,32,40)$, no other seismic phase is predicted to arrive this late in the seismogram with similar move-out and polarization. Love waves travelling along the surface would have similar polarization, but arrive much earlier and show a large move-out and dispersion. Multiply-reflected body waves should also arrive earlier. We therefore interpreted this signal as an S-wave reflection from the core-mantle-boundary (CMB) that, when using seismic velocity models from (32), corresponds to a core with a radius in the range 1770 to $1890 \mathrm{~km}$. We also searched for other phases such as core-reflected $\mathrm{P}$-waves (PcP), but were unable to find consistent arrivals. This was not unexpected due to the lower $\mathrm{P}$-wave reflection coefficient at the $\mathrm{CMB}$. A shear wave that is converted into a $\mathrm{P}$-wave at the CMB ( $\mathrm{ScP})$ is predicted to arrive 290 seconds after $\mathrm{P}$ for $\mathrm{S} 0325 \mathrm{a}$ and we observed a weak phase by correlation analysis. This arrival is only 2 seconds after the identified SSS arrival reported in (32), so the peak, while consistent, is not used for inversion here. For four of the six events we investigated, (32) reports a second arrival within 20 seconds after the main S-wave. We considered these secondary arrivals to be the depth phase (sS), resulting in marsquakes that occur in the depth range $20-35 \mathrm{~km}$. 
The spectral character and the travel time of the direct S-wave for the most distant event (S0167b) located to date $(30,32)$ was consistent with and comparable to that of the ScS phase (Fig. S6-1) (38). This supports the identification of the latter as a core-reflection that has traversed the entire attenuating mantle. The attenuation-corrected power of the $\mathrm{ScS}$ phases is below that of the corresponding S-wave arrivals by 10-20 dB. This value is slightly below the ray-theoretical prediction from geometrical spreading (Fig. S6-2) (38), assuming full reflection of SH waves at the $\mathrm{CMB}$, possibly due to scattering at other interfaces and three-dimensional structure.

With the identification of the ScS phase, we picked arrival times of the peak energy for each event (Table 1) and inverted those together with the arrival times of P, S, PP, SS, PPP, SSS from (32) for mantle P- and S-wave velocity and density, epicentral distance for all considered events, and radius and mean density of the core. We conducted three separate inversions that relied on a pure seismic parameterization, and two mineralogically constrained inversions: a geodynamic $(41,42)$ and a geophysical parameterization (4). The seismic parameterization considers a layered model of Mars described by P- and S-wave velocity gradients, respectively. The geodynamic parameterization depends on quantities that influence the thermo-chemical evolution of the planet and accounts for $4.5 \mathrm{Gyr}$ of planetary evolution. The geophysical parameterization relies on a unified description of phase equilibria, seismic properties, and thermo-chemical parameters. The parameterizations (38) reflect, in going from seismic over geodynamic to geophysical parameterization, a decrease in the number of degrees of freedom, as the two latter parameterizations depend increasingly on mineral physics information, and therefore better resolved parameters. In the geophysical and geodynamic inversions, we included geodetic data in the form of the degree-2 Love number (11), mean density (16), and mean moment of inertia (11). Because the mean density of the core depends on that of the mantle and therefore on the bulk mantle composition, we considered six different model Martian compositions $(12,14,43-47)$ as part of the geophysical inversion. To solve the inverse problem, we employed a stochastic algorithm (48) that samples models fitting the differential body wave travel times within uncertainties and are consistent with prior information (38).

We plotted the results from the joint inversion of the differential body wave travel times and the geophysical data (Fig. 2). The S-wave velocity profiles (Fig. 2A) we obtained from the three parameterizations were found to be in good agreement. More scatter exists in the P-wave velocity profiles, which reflects fewer P-wave observations, and structure is only constrained to $800 \mathrm{~km}$ depth (Fig. 2A). All parameterizations provide a good fit to the ScS-P travel time observations (Fig. 2B). Above $800 \mathrm{~km}$ depth, the velocity profiles are similar to those obtained by the upper mantle inversion (32) and below, the S-wave velocity profiles show a distinct increase around $1050 \mathrm{~km}$ depth, equivalent of the " $410-\mathrm{km}$ " seismic discontinuity in Earth's mantle that marks the onset of the mantle transition zone, where the dominant upper mantle mineral olivine transforms to wadsleyite. The core-mantle-boundary occurs between 1520-1600 $\mathrm{km}$ depth, corresponding to core-mantle-boundary pressures of 18-19 $\mathrm{GPa}$ and temperatures in the range $\sim 1900-2000 \mathrm{~K}$. These conditions are unfavorable for the stabilization of bridgmanite, and implies that the lower mantle of Mars is mineralogically comparable to Earth's mantle transition zone. This means that a relatively dense and thermally insulating lower mantle is absent in Mars, which favors the development of an early thermally-driven dynamo as a means of explaining crustal magnetism because of elevated core heat flux $(1,49,50)$. 
In agreement with geodetic observations that require a liquid core (10), the observation of $\mathrm{ScS}$ with significant relative amplitudes compared to direct $\mathrm{S}$-waves rules out a solid outer core, because reflection coefficients would be too small at a solid-solid interface (fig. S6-2, (38)). The separate inversions converge on the same mean radius but show more spread in mean core density (Fig. 2C), which reflects the trade-off with mantle density through bulk mantle composition (indicated by the blue circles in Fig. 1C). Based on the distributions, we estimate core radius to be $1830 \pm 40 \mathrm{~km}$, at the upper end of pre-mission estimates $(4,8,15,16)$ that were based on an earlier and slightly lower degree-2 Love number $(0.169 \pm 0.006,(51))$, while mean core density lies in the range $5.7-6.3 \mathrm{~g} / \mathrm{cm}^{3}$. We also conducted separate inversions using the geodynamic method to consider the influence of individual data sets on the retrieved core properties (38). These inversions showed that the mean core radius changed from $1836 \mathrm{~km}$ (seismic data only) to $1815 \mathrm{~km}$ (geodetic data only), while the mean core density remained unchanged. To test the influence of the source depth, we fixed it to $50 \mathrm{~km}$ for all events, consistent with $(29,32)$, and found that this would change the core radius to $1820 \pm 40 \mathrm{~km}$, which is similar to the above range.

Compositional constraints on the core typically derive from geochemical models coupled with metal-silicate partitioning and mass balance arguments (52-55), but depend on the assumed compositions of the building blocks $(56,57)$. While $\mathrm{S}$ is commonly considered the main light element $(14,44,45,55)$ because of its abundance in the mantle as determined from the Martian meteorites (58) and its siderophile nature at the P-T- $f \mathrm{O}_{2}$ conditions of the formation of Mars's core (59), additional light elements, including $\mathrm{C}, \mathrm{O}, \mathrm{Si}, \mathrm{N}$, and $\mathrm{H}$ are all potentially viable candidates as in Earth's core (60-63). Ni is also expected to be a core constituent based on meteorite compositions (64) and should make up 5-6 wt\% (4). The purple-shaded areas in Fig. $2 \mathrm{C}$ indicate how the mean core density varies with $\mathrm{S}$ content in the Fe-S, Fe-S-O, Fe-S-O-H, and Fe-S-O-H-C systems, based on thermodynamic solution models constructed using experimental data (65-68). For a core composed of Fe and $\mathrm{S}$, sulfur contents surpass $25 \mathrm{wt} \%$, which is above the value of the sulfur-richest meteorites (EH chondrites) and in excess of what is deduced from geo- and cosmochemical models $(<13-19$ wt.\%, $(52,55))$. To bring the $\mathrm{S}$ content in line with the cosmochemical constraints, additional light elements (e.g., C, O, H) in the core are needed, see $\mathrm{S} 11$ (38). For geochemically defensible amounts of $\mathrm{S}, \mathrm{O}$, and $\mathrm{H}$, the mean core density has to be $>6 \mathrm{~g} / \mathrm{cm}^{3}$, which encompasses the upper end of our predictions (Fig. 2C). Because our core density is tied to reasonable assumptions about the composition and temperature of the Martian mantle, a higher mean core density is possible, that requires less light elements as a result of a lower bulk mantle FeO content (46) or higher temperatures as seen in some geodynamic models (69). The influence of a lower bulk mantle $\mathrm{FeO}$ content on mean core density is reflected by the cloud of blue circles with a mean core density $>6.1 \mathrm{~g} / \mathrm{cm}^{3}$ (Fig. $2 \mathrm{C}$ ) that were obtained on the basis of the bulk mantle composition of (46). Thus a lower bulk mantle FeO content seems to provide a better match than the canonical Martian compositions with $\mathrm{FeO}$ contents exceeding 17 wt $\%(12,14,44,45)$, which had also found to be at odds with geophysical constraints $(4,57)$. As a preliminary observation, our results can be construed as pointing to an $\mathrm{Fe}-\mathrm{Ni}$ core that is composed of, in order of abundance, S (10-15 wt.\%), O ( $<5 \mathrm{wt.} \%)$, and $\mathrm{H}$ and $\mathrm{C}(<1 \mathrm{wt} . \%$ level) (38). While such $\mathrm{O}, \mathrm{H}$, and $\mathrm{C}$ contents represent upper limits, they serve to emphasize the need for light elements in Mars' core. 
A chemical composition close to the eutectic ( $\sim 15$ wt. $\% \mathrm{~S}$ in the Fe-FeS binary at the coremantle-boundary conditions of Mars) likely prevented crystallization of a bottom-up inner core because of the temperatures $(\sim 1200 \mathrm{~K})$ required to drop below the liquidus $(70)$. This is compatible with the absence of a current Martian geodynamo (71). Strong crustal remnant magnetization in the southern hemisphere of Mars (72) and observations of further magnetized units suggest a dynamo that was active between $4.5 \mathrm{Ga}$ and $3.7 \mathrm{Ga}(9)$. The dynamo would have been thermally-driven in the first few hundred Myr $(49,50)$ and possibly followed by a compositionally-driven dynamo that may resuscitate through $\mathrm{FeO}$ exsolution (73) or inner-core crystallization $(71,74,75)$. This, however, depends critically on the light element content and thermal state of the core.

As a consequence of the large core, the seismic core shadow on Mars (Fig. 3) commences at closer epicentral distances $\left(94^{\circ}-98^{\circ}\right)$ than on Earth $\left(100^{\circ}(76)\right)$. This makes up half of the planet, including $33 \%$ of total extensional faults with $75 \%$ of those in terrain younger than $600 \mathrm{Ma}(77)$. For Marsquakes in Tharsis, the region of Mars presumed to host most recent tectonic activity (78), direct P- and S-waves are therefore unobservable. That these events can therefore not be located easily, may result in an underestimation of the global seismic activity of Mars as seen from InSight's location (27).

The differentiation of Mars into a primordial crust, mantle, and core is likely the result of early magma ocean crystallization and solidification that could potentially result in compositional stratification of the mantle $(69,79)$. There is, however, no direct evidence for this based on current observations. Continued analysis using marsquakes observed during the extended mission will be required to delve into the question of deep mantle layering. To determine the compressional wave speeds of the core itself, and further refine light element content, an unequivocal observation of an event beyond the core shadow, with clear core-crossing phases like PKP or SKS will be necessary. This would allow us to employ velocity-density systematics (61) as a means of gaining further insight into the total light element content of the core. In the interim, the new Martian seismic data and models presented here provide a wealth of new insights into the interior structure of Mars, which contain the clues needed to unravel the planetary building blocks (57), the physical and chemical conditions during assembly (52) and chronology of crust, mantle, and core formation (25).

\section{Materials and methods:}

The supplementary material consists of four main parts:

I. Sections 1-5, which present several complementary efforts to detect and characterize ScS arrivals;

II. Sections 6-7, which combines the $\mathrm{ScS}$ analyses and characterizes the corresponding environmental noise levels;

III. Sections 8-10, which describe the inversion for structural models;

IV. Section 11, which describes the implications of the structural models for Martian core composition.

References (88-124) 


\section{References and Notes}

1. D. J. Stevenson, Nature. 412, 214-219 (2001).

2. M. T. Zuber et al., Science. 287, 1788-1793 (2000).

3. C. M. Bertka, Y. Fei, Earth Planet. Sci. Lett. 157, 79-88 (1998).

4. A. Khan et al., J. Geophys. Res. Planets. 123, 1-37 (2018).

5. D. Breuer, D. A. Yuen, T. Spohn, Earth Planet. Sci. Lett. 148, 457-469 (1997).

6. P. van Thienen, N. J. Vlaar, A. P. van den Berg, Phys. Earth Planet. Inter. 142, 61-74 (2004).

7. T. Ruedas, P. J. Tackley, S. C. Solomon, Phys. Earth Planet. Inter. 216, 32-58 (2013).

8. S. E. Smrekar et al., Space Sci. Rev. 215, 3-3 (2019).

9. A. Mittelholz, C. L. Johnson, J. M. Feinberg, B. Langlais, R. J. Phillips, Sci. Adv. 6, eaba0513 (2020).

10. C. F. Yoder, A. S. Konopliv, D. N. Yuan, E. M. Standish, W. M. Folkner, Science. 300, 299-303 (2003).

11. A. S. Konopliv et al., Geophys. Res. Lett., 0-2 (2020).

12. G. Dreibus, H. Wänke, in Proceedings of the 27th International Geological Congress (1984), vol. 11, pp. 120.

13. H. Y. McSween, K. Keil, Geochim. Cosmochim. Acta. 64, 2155-2166 (2000).

14. G. J. Taylor, Chem. Erde - Geochem. 73, 401-420 (2013).

15. A. Bagheri, A. Khan, D. Al-Attar, O. Crawford, D. Giardini, J. Geophys. Res. Planets. 124, 2703-2727 (2019).

16. A. Rivoldini, T. Van Hoolst, O. Verhoeven, A. Mocquet, V. Dehant, Icarus. 213, 451-472 (2011).

17. C. Hutton, Philos. Trans. R. Soc. Lond. (1778).

18. R. D. Oldham, Q. J. Geol. Soc. 62, 456-475 (1906).

19. I. Lehmann, Bur. Cent. Séismologique Int. Sér. Trav. Sci. 14, 87-115 (1936).

20. K. E. Bullen, Phys. Chem. Earth. 1, 68-93 (1956).

21. C. G. Dahm, Eos Trans. Am. Geophys. Union. 15, 80-83 (1934).

22. G. V. Latham et al., Science. 174, 687-92 (1971).

23. R. F. Garcia, J. Gagnepain-Beyneix, S. Chevrot, P. Lognonné, Phys Earth Planet Inter. 188, 96-113 (2011).

24. R. C. Weber, P.-Y. Lin, E. J. Garnero, Q. Williams, P. Lognonne, Science. 331, 309-312 (2011).

25. N. Dauphas, A. Pourmand, Nature. 473, 489-492 (2011).

26. K. Mezger, V. Debaille, T. Kleine, Space Sci. Rev. 174, 27-48 (2013). 
27. W. B. Banerdt et al., Nat. Geosci. 13, 183-189 (2020).

28. P. Lognonné et al., Space Sci. Rev. 215, 12-12 (2019).

29. D. Giardini et al., Nat. Geosci. 13, 205-212 (2020).

30. InSight Marsquake Service, Mars Seismic Catalogue, InSight Mission; V6 2021-04-01 (2021), p. 12 MB, , doi:10.12686/A11.

31. P. Lognonné et al., Nat. Geosci. 13, 213-220 (2020).

32. A. Khan et al., Science. in revisio (2021).

33. B. Knapmeyer-Endrun, M. P. Panning, et al., Science. in revision.

34. J. Taylor, N. A. Teanby, J. Wookey, J. Geophys. Res. E Planets. 118, 2570-2581 (2013).

35. N. Brinkman et al., J. Geophys. Res. Planets (2021), doi:10.1029/2020je006546.

36. M. Schimmel, J. Gallart, Bull. Seismol. Soc. Am. 94, 1016-1035 (2004).

37. P. S. Schultz, J. F. Claerbout, GEOPHYSICS. 43, 691-714 (1978).

38. "Materials and methods are available as supplementary materials at the Science website."

39. J.-R. Scholz et al., Earth Space Sci. 7, 1-31 (2020).

40. A.-C. Plesa et al., J. Geophys. Res. Planets, in press, doi:10.1029/2020JE006755.

41. M. Drilleau, H. Samuel, A. Rivoldini, M. Panning, P. Lognonné, Geophys. J. Int. (2021), doi:10.1093/gji/ggab105.

42. H. Samuel, P. Lognonné, M. P. Panning, V. Lainey, Nature. 569, 523-527 (2019).

43. A. Khan, J. A. D. Connolly, J. Geophys. Res. 113, E07003-E07003 (2008).

44. K. Lodders, B. Fegley, Icarus. 126, 373-394 (1997).

45. C. Sanloup, A. Jambon, P. Gillet, Phys. Earth Planet. Inter. 112, 43-54 (1999).

46. T. Yoshizaki, W. F. McDonough, Geochim. Cosmochim. Acta. 273, 137-162 (2020).

47. C. B. Agee, D. S. Draper, Earth Planet. Sci. Lett. 224, 415-429 (2004).

48. K. Mosegaard, A. Tarantola, J. Geophys. Res. 100, 12431-12431 (1995).

49. D. Breuer, T. Spohn, J. Geophys. Res. 108, 5072-5072 (2003).

50. J.-P. Williams, F. Nimmo, Geology. 32, 97-97 (2004).

51. A. S. Konopliv, R. S. Park, W. M. Folkner, Icarus. 274, 253-260 (2016).

52. M. C. Brennan, R. A. Fischer, J. C. E. Irving, Earth Planet. Sci. Lett. 530, 115923 (2020).

53. K. Righter, N. L. Chabot, Meteorit. Planet. Sci. 46, 157-176 (2011). 
54. D. C. Rubie et al., Icarus. 248, 89-108 (2015).

55. E. S. Steenstra, W. van Westrenen, Icarus. 315, 69-78 (2018).

56. C. Fitoussi, B. Bourdon, X. Wang, Earth Planet. Sci. Lett. 434, 151-160 (2016).

57. C. Liebske, A. Khan, Icarus. 322, 121-134 (2019).

58. G. Dreibus, H. Wanke, Meteoritics. 20, 367-381 (1985).

59. N. Rai, W. van Westrenen, J. Geophys. Res. Planets. 118, 1195-1203 (2013).

60. J. Badro, J. P. Brodholt, H. Piet, J. Siebert, F. J. Ryerson, Proc. Natl. Acad. Sci. 112, 12310-12314 (2015).

61. F. Birch, J. Geophys. Res. 1896-1977. 69, 4377-4388 (1964).

62. J.-P. Poirier, Phys. Earth Planet. Inter. 85, 319-337 (1994).

63. V. N. Zharkov, Sol. Syst. Res. 30, 456 (1996).

64. A. E. Ringwood, Geochem. J. 11, 111-135 (1977).

65. J. V. Badding, H. K. Mao, R. J. Hemley, (American Geophysical Union, 2013), pp. 363-371.

66. T. Komabayashi, J. Geophys. Res. Solid Earth, 2014JB010980 (2014).

67. G. Morard et al., Am. Mineral. (2018).

68. H. Terasaki et al., J. Geophys. Res. Planets. 124 (2019).

69. L. T. Elkins-Tanton, E. M. Parmentier, P. C. Hess, Meteorit. Planet. Sci. 38, 1753-1771 (2003).

70. Y. Mori et al., Earth Planet. Sci. Lett. 464, 135-141 (2017).

71. A. J. Stewart, M. W. Schmidt, W. Van Westrenen, C. Liebske, Science. 316, 1323-1325 (2007).

72. M. H. Acuña et al., Science. 284, 790-793 (1999).

73. K. Tsuno, E. Ohtani, H. Terasaki, Phys. Earth Planet. Inter. 160, 75-85 (2007).

74. D. Breuer, W. B. Moore, in Treatise on Geophysics (Second Edition), G. Schubert, Ed. (Elsevier, Oxford, Second Edi., 2015), pp. 255-305.

75. D. J. Hemingway, P. E. Driscoll, J. Geophys. Res. Planets, in press, doi:https://doi.org/10.1029/2020JE006663.

76. B. L. N. Kennett, E. R. Engdahl, Geophys. J. Int. 105, 429-465 (1991).

77. M. Knapmeyer et al., J. Geophys. Res. E Planets. 111, 1-23 (2006).

78. R. C. Anderson et al., J. Geophys. Res. Planets. 106, 20563-20585 (2001).

79. H. Samuel et al., J. Geophys. Res. Planets, in press, doi:https://doi.org/10.1029/2020JE006613.

80. A. G. Marusiak, N. C. Schmerr, M. E. Banks, I. J. Daubar, Icarus. 335, 113396 (2020). 
81. D. E. Smith et al., J. Geophys. Res. Planets. 106, 23689-23722 (2001).

82. M. Böse et al., Bull. Seismol. Soc. Am. (2021), doi:10.1785/0120210045.

83. J. D. Hunter, Comput. Sci. Eng. 9, 90-95 (2007).

84. L. Krischer et al., Comput. Sci. Discov. 8, 014003-014003 (2015).

85. C. R. Harris et al., Nature. 585, 357-362 (2020).

86. P. Virtanen et al., Nat. Methods. 17, 261-272 (2020).

87. InSight Mars SEIS Data Service, SEIS raw data, Insight Mission (IPGP, JPL, CNES, ETHZ, ICL, MPS, ISAE-Supaero, LPG, MFSC, 2019; http://datacenter.ipgp.fr/networks/detail/XB_2016).

88. S. C. Stähler, A. Khan, M. Drilleau, A. C. Duran, H. Samuel, Interior Models of Mars from inversion of seismic body waves (2021), (available at doi:10.18715/IPGP.2021.kpmqrnz8).

89. J. F. Clinton et al., Space Sci. Rev. 214, 133-133 (2018).

90. R. G. Stockwell, L. Mansinha, R. P. Lowe, IEEE Trans. Signal Process. 44, 998-1001 (1996).

91. S. Greenhalgh, D. Sollberger, C. Schmelzbach, M. Rutty, in Advances in Geophysics, C. Schmelzbach, Ed. (Elsevier, 2018; https://www.sciencedirect.com/science/article/pii/S0065268718300025), vol. 59, pp. 123170.

92. J. C. Samson, Geophys. J. Int. 72, 647-664 (1983).

93. E. Stutzmann et al., J. Geophys. Res. Planets. 126 (2021), doi:10.1029/2020JE006545.

94. J. Park, F. L. Vernon, C. R. Lindberg, J. Geophys. Res. Solid Earth. 92, 12664-12674 (1987).

95. K. D. Koper, V. L. Hawley, Earthq. Sci. 23, 439-447 (2010).

96. P. J. Goodling, V. Lekic, K. Prestegaard, Earth Surf. Dyn. 6, 351-367 (2018).

97. E. Galetti, A. Curtis, Tectonophysics. 532-535, 1-26 (2012).

98. M. Schimmel, Bull. Seismol. Soc. Am. 89, 1366--1378 (1999).

99. J. F. Clinton et al., Phys. Earth Planet. Inter. 310 (2021), doi:10.1016/j.pepi.2020.106595.

100. M. M. Haney et al., Bull. Volcanol. 82, 18 (2020).

101. N. Compaire et al., J. Geophys. Res. Planets, in press, doi:https://doi.org/10.1029/2020JE006498.

102. M. Schimmel, E. Stutzmann, J. Gallart, Geophys. J. Int. 184, 494-506 (2011).

103. S. Ceylan et al., Phys. Earth Planet. Inter. 310, 106597-106597 (2021).

104. J. F. Montalbetti, E. R. Kanasewich, Geophys. J. Int. 21, 119-129 (1970).

105. S. Rost, C. Thomas, Rev. Geophys. 40, 1008-1008 (2002).

106. J. F. Clinton et al., Seismol. Res. Lett. 88, 1290-1302 (2017). 
107. G. Prieto, R. L. Parker, F. L. Vernon, Comput. Geosci. 35, 1701-1710 (2009).

108. J. N. Brune, J. Geophys. Res. 75, 4997-5009 (1970).

109. G. Nolet, A breviary of seismic tomography: imaging the interior of the earth and sun (Cambridge University Press, 2008; www.cambridge.org/9780521882446).

110. C. Charalambous et al., J. Geophys. Res. Planets, in press, doi:10.3929/ethz-b-000479669.

111. R. F. Garcia et al., J. Geophys. Res. Planets, in press, doi:https://doi.org/10.1029/2019JE006278.

112. J. a. D. Connolly, Geochem. Geophys. Geosystems. 10 (2009), doi:https://doi.org/10.1029/2009GC002540.

113. L. Stixrude, C. Lithgow-Bertelloni, Geophys. J. Int. 162, 610-632 (2005).

114. L. Stixrude, C. Lithgow-Bertelloni, Geophys. J. Int. 184, 1180-1213 (2011).

115. J. A. D. Connolly, A. Khan, Geophys. Res. Lett. 43, 5026-5034 (2016).

116. M. Drilleau et al., Earth Space Sci., in press, doi:https://doi.org/10.1029/2020EA001118.

117. S. $\sim$ R. Taylor, S. McLennan, Planetary Crusts: Their Composition, Origin and Evolution (2009).

118. J. A. D. Connolly, Earth Planet. Sci. Lett. 236, 524-541 (2005).

119. A. Khan, K. Mosegaard, J. G. Williams, P. Lognonné, J. Geophys. Res. Planets. 109, 9007-9007 (2004).

120. H. P. Crotwell, T. J. Owens, J. Ritsema, Seismol. Res. Lett. 70, 154-160 (1999).

121. K. Tsuno, D. S. Grewal, R. Dasgupta, Geochim. Cosmochim. Acta. 238, 477-495 (2018).

122. I. Blanchard, J. Badro, J. Siebert, F. J. Ryerson, Earth Planet. Sci. Lett. 427, 191-201 (2015).

123. J. G. O’Rourke, S.-H. Shim, J. Geophys. Res. Planets. 124, 3422-3441 (2019).

124. Y. Shibazaki, E. Ohtani, H. Terasaki, A. Suzuki, K. Funakoshi, Earth Planet. Sci. Lett. 287, $463-470$ (2009).

125. G. Morard et al., J. Geophys. Res. Solid Earth. 122, 7813-7823 (2017).

\section{Acknowledgments:}

This is InSight contribution 200.

\section{Funding:}

S.C.S., A.K., D.G., J.C., A.C.D., G.Z., and N.D. acknowledge support from ETHZ the ETH+ funding scheme (ETH+2 19-1: "Planet MARS").

W.B.B., A.G.M., M.P.P., and S.E.S. were supported by the NASA InSight mission and funds from the Jet Propulsion Laboratory, California Institute of Technology, under a contract with the National Aeronautics and Space Administration (80NM0018D0004).

D.A. has received funding from the European Research Council (ERC) under the European Union's Horizon 2020 research and innovation Programme (Grant agreement 724690). 
The French teams acknowledge support from CNES as well as Agence Nationale de la Recherche (ANR-14-CE36-0012-02 and ANR-19-CE31-0008-08).

A. R. was financially supported by the Belgian PRODEX program managed by the European Space Agency in collaboration with the Belgian Federal Science Policy Office.

M.S. wishes to thank SANIMS (RTI2018-095594-B-I00).

M.V.D. received support from the European Research Council (ERC) under the EU's Horizon 2020 programme (grant No. 714069).

D.S. and C.S. acknowledge funding from ETH Research grant ETH-06 17-02.

J.C.E.I. acknowledges support from NASA grant 80NSSC18K1633.

N.S, D.K., Q.H., R.M., V.L., and A.G.M. acknowledge NASA grant 80NSSC18K1628 for support.

V.L. acknowledges support from the Packard Foundation.

A.H. was funded by the UK Space Agency (grant ST/R002096/1).

A.-C.P. acknowledges the financial support and endorsement from the DLR Management Board Young Research Group Leader Program and the Executive Board Member for Space Research and Technology.

\section{Author contributions:}

S.C.S, D.G. S.C, R.G., Q.H., D.K. V.L., M.S., N.S., D.S., É.S., C.S., G.Z. analyzed the seismic data and made arrival time picks.

S.C.S., P.L., D.G., Z.X., C.C. and W.T.P. performed the statistical analysis of the observed signals.

S.C.S., Q.H., N.S., R.M. and A.G.M. identified the arrivals as ScS based on interior models from A.K., H. S. and A.R..

A.K., M.D., A.C.D. and H.S. performed the inversions.

S.C.S., A.K., P.L., D.G., D.A., J.C.E.I., M.K., C.P. A-C.P., A.R., T.G., and S.E.S. participated and contributed to the interpretation of the results.

Review of the continuous data and detection of marsquakes was done by S.C.S., S.C., G.Z, N.D., J.F.C., M.P. and A.H. with operational support by É.B. and P.D..

S.C.S. and A.K. wrote the paper with contributions from H.S., N.S., D.A., J.C.E.I., A.G.M., AC.P., A.R., J.C. and M.v.D.

J.C.E.I., R.M., M.K. and V.L. reviewed the contributions to the supplementary material.

The InSight mission is managed by W.B.B., M.P.P., and S.E.S.

The SEIS instrument development was led by P.L., D.G., and W.T.P.

Supplementary section 1 was written by M.S., D.S. and É.S. with contributions from S.C.S., C.S., and Z.X.

Supplementary section 2 was written by D.K. and V.L. with contributions from J.C.E.I., and N.S. 
Supplementary section 3 was written by M.S. and É.S.

Supplementary section 4 was written by R.G. with contributions from M.D.

Supplementary section 5 was written by Q.H. with contributions from N.S.

Supplementary section 6 was written by S.C.S. with contributions from the authors of the other supplements.

Supplementary section 7 was written by Z.X. and C.C. with contributions from P.L. and W.T.P.

Supplementary section 8 was written by A.K., M.D, A.C.D., and H.S.

Supplementary section 9 was written by M.D.

Supplementary section 10 was written by A.C.D, A.K., and M.D.

Supplementary section 11 was written by D.A. and A.R, with contributions from A.K.

Competing interests: Authors declare that they have no competing interests. 


\section{Figures}
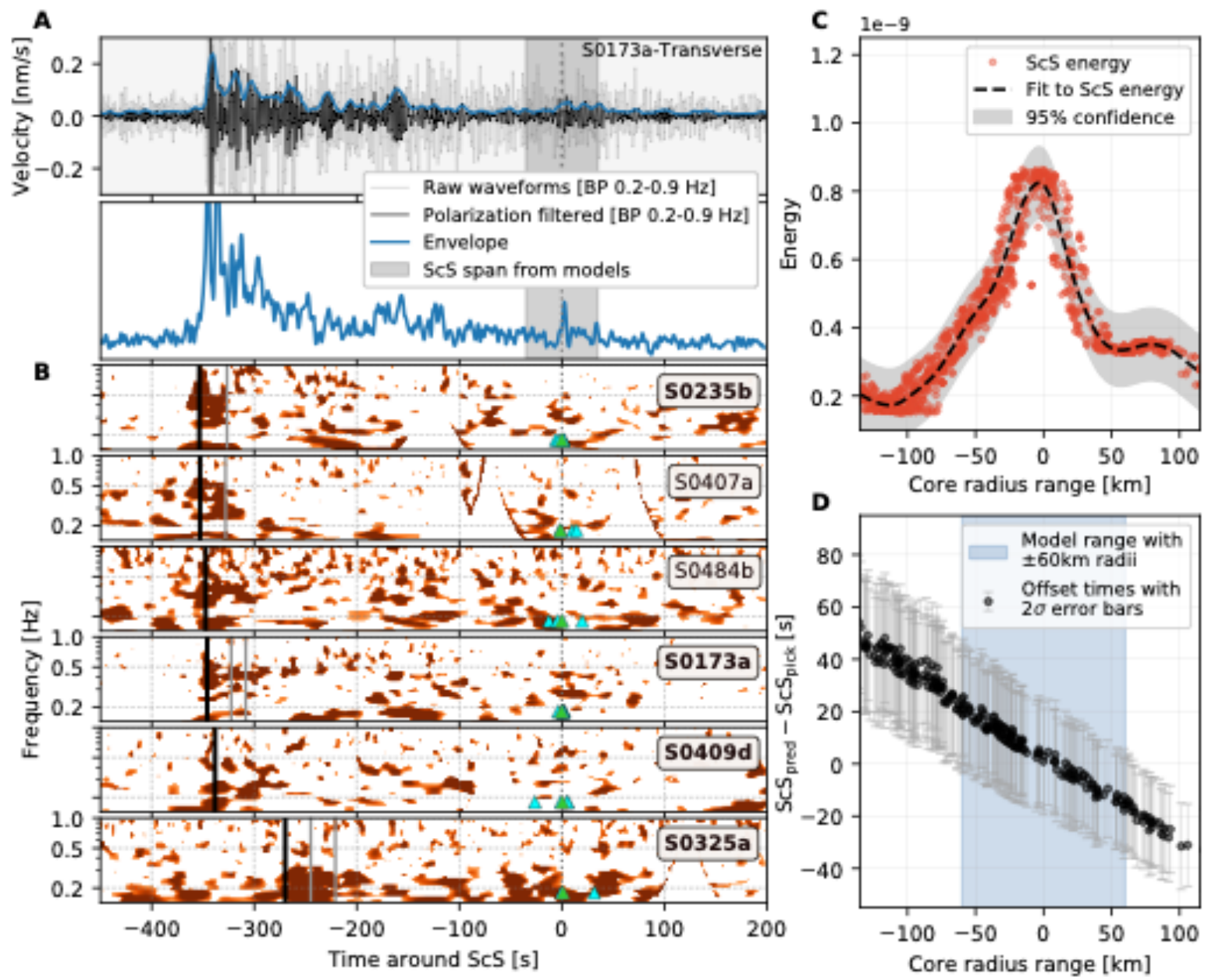

Fig. 1. Summary of data processing and identification of core-reflected S-waves.

(A) Seismogram and envelope of event S0173a before and after polarization filtering (top) and envelope stack for all six events (bottom). The individual event envelopes are shown in Fig. S1-4 (38). (B) Polarization-filtered spectrograms for each of the six events considered here. Cyan triangles mark proposed $\mathrm{ScS}$ picks based on the processing methods described in the main text, whereas green triangles mark the final set of picks summarized in Table 1. Light grey lines mark SS and SSS picks from (32). All events show energy around the predicted arrival time of ScS, using a model from (32) with a core radius of $1830 \mathrm{~km}$, in agreement with the ScS observation for S0173a shown in (A). Bold event labels (e.g., S0235b) indicate events with strong ScS energy. (C) Stacked energy in a $10 \mathrm{~s}$ time window around $\mathrm{ScS}$ as predicted for 5000 models from (32) with core radii centered around 1830. (D) Residual travel times of the models presented in 
(C) compared to the picks in (B), show that the ScS picks are able to constrain the core radius to within $\pm 60 \mathrm{~km}(80)$.
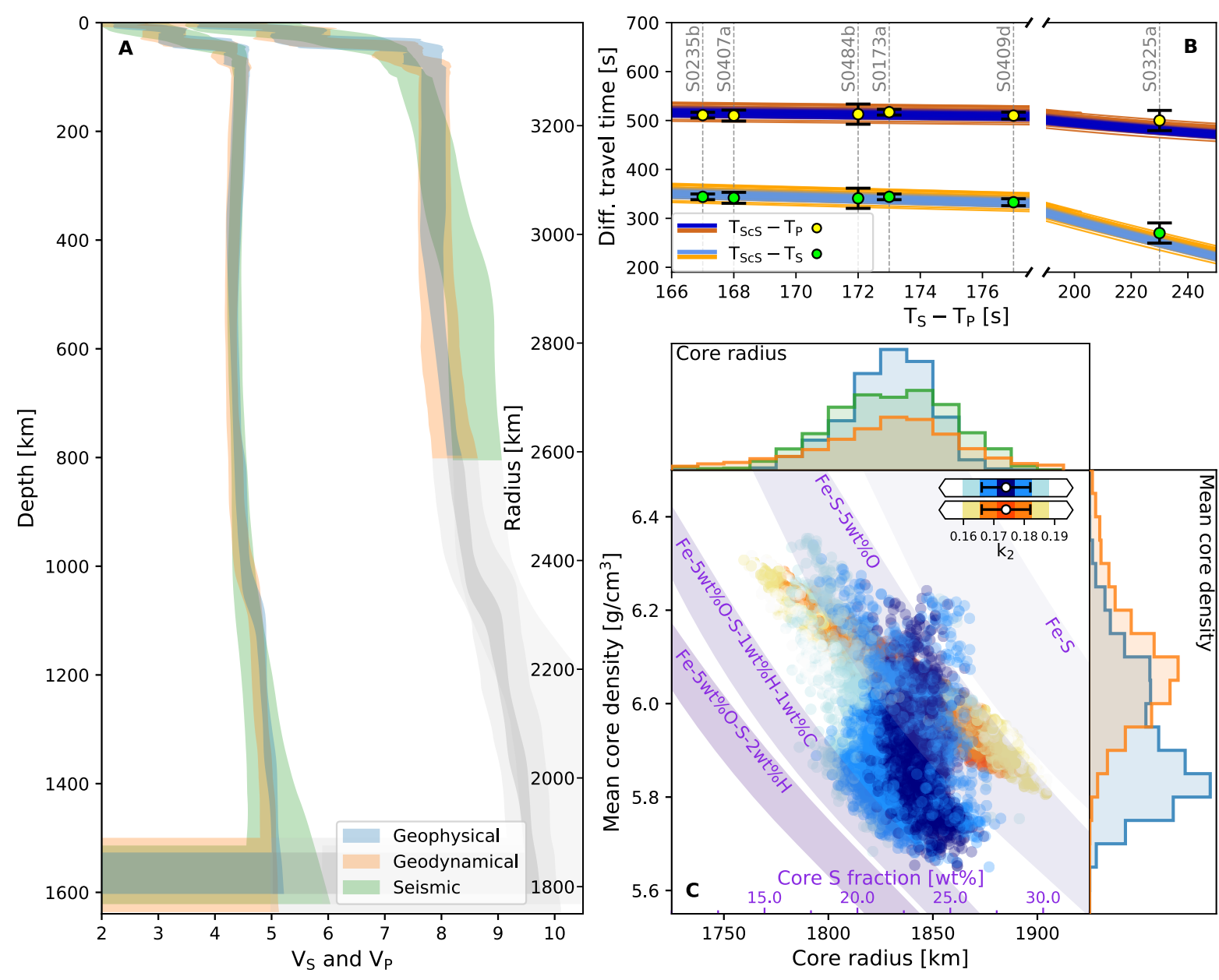

Fig. 2. Mars's mantle and core structure. (A) Inverted seismic wave velocity profiles (95\% credible intervals) based on the three inversion methods. The pale gray-shaded area below 800 $\mathrm{km}$ depth for the P-wave velocity profiles indicates that no direct information is available for this region. (B) Differential body wave travel time misfits for all sampled models obtained from the geophysical (blue shades) and geodynamical (orange shades) inversions shown in panel (A). Yellow and green circles indicate the observations including error bars, respectively. A detailed version of the misfit plot is shown in Figure S9-1 (38). (C) Sampled core properties. Middle plot: mean core density versus core radius for the geophysical (blue) and geodynamical (orange) methods, while their marginal distributions are shown as histograms to the right and on the top. The seismic method only constrains core radius. The blue and orange models are color-coded according to their fit to the tidal response in the form of the observed degree-2 Love number $k_{2}$ (11) defined by the white circles and horizontal error bars. Relying on Fe-S models, the purple bands indicate the variation of core sulfur (S) content (purple axis) with mean core density for four different iron $(\mathrm{Fe})$ - light element $(\mathrm{S}, \mathrm{O}, \mathrm{H}$, and $\mathrm{C})$ assemblages $(65-68)$. 


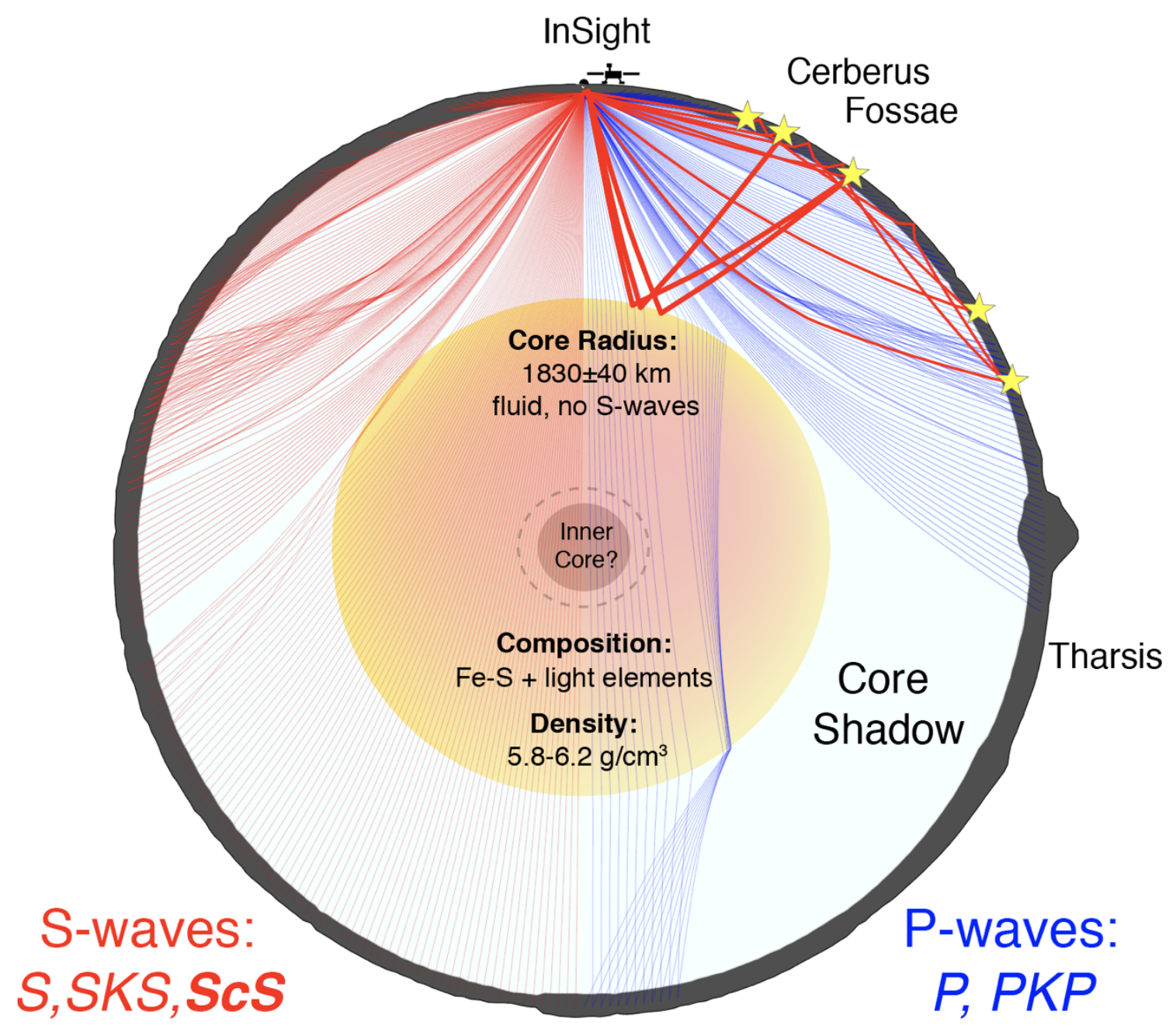

Fig. 3. Schematic diagram of Mars's interior structure. The cross section depicts the coreinduced shadow zone for seismic waves. The surface topography is a cut through the MOLA map (81) on a great circle arc from InSight through Olympus Mons. The S-wave shadow zone is minimal and probably filled by diffracted S-waves (Sdiff), while the P-wave shadow zone is significant and contains specifically the Tharsis region. The existence of an inner core cannot be determined by current data and the seismic ray paths shown assume no inner core. Topography and InSight lander are exaggerated in scale. 


\begin{tabular}{|l|l|l|l|l|l|}
\hline Event & tS-tP $[\mathrm{sec}]$ & tScS - tP $[\mathrm{sec}]$ & sigma(tScS) & depth $[\mathrm{km}]$ & $\mathrm{M}_{\mathrm{w}}$ \\
\hline S0235b & 167 & 511 & 3 & $24 \pm 5$ & 3.5 \\
\hline S0407a & 168 & 510 & 10 & $25 \pm 5$ & 3.0 \\
\hline S0484b & 172 & 513 & 20 & $33 \pm 5$ & 2.9 \\
\hline S0173a & 173 & 512 & 3 & $24 \pm 5$ & 3.6 \\
\hline S0409d & 177 & 510 & 5 & $25 \pm 5$ & 3.1 \\
\hline S0325a & 230 & 500 & 20 & $30 \pm 5$ & 3.7 \\
\hline
\end{tabular}

Table 1: Consolidated pick times of $P$ and $\mathrm{SeS}$ for the events used in this study. Magnitudes are from the MQS catalog v6 (30). Depth estimates are based on the identification of the depth phase sS (see the main text). The events are labelled by mission Sol of occurrence and sub-labeled alphabetically for Sols with more than 1 event. (82)

We acknowledge NASA, CNES, partner agencies and Institutions (UKSA, SSO, DLR, JPL, IPGP-CNRS, ETHZ, IC, MPS-MPG) for the development of SEIS. The Swiss contribution in implementation of the SEIS electronics was made possible through funding from the federal Swiss Space Office (SSO), the contractual and technical support of the ESA-PRODEX office. Numerical simulations were supported by a grant from the Swiss National Supercomputing Centre (CSCS) under project ID s922, as well as HPC resources of CINES under the allocation A0090407341, made by GENCI. We thank Boris Dintrans, director of CINES, for his efficient handling of our request for computational time. Figures were created using matplotlib (83), seismic data processing in ObsPy (84), numerical evaluation in NumPy and SciPy $(85,86)$. Data and materials availability: We thank the operators of JPL, SISMOC, MSDS, IRIS-DMC and PDS for providing SEED SEIS data (87). 300 interior models derived in this study are available as electronic supplement (38) and from (88).r 1 


\section{Supplementary Materials}

\section{Science МIAAS}

\section{Supplementary Materials for}

\section{Seismic detection of the Martian core}

Simon C. Stähler, Amir Khan, W. Bruce Banerdt, Philippe Lognonné, Domenico Giardini, Savas Ceylan, Mélanie Drilleau, A. Cecilia Duran, Raphaël F. Garcia, Quancheng Huang, Doyeon Kim, Vedran Lekic, Henri Samuel, Martin Schimmel, Nicholas Schmerr, David Sollberger, Éléonore Stutzmann, Zongbo Xu, Daniele Antonangeli, Constantinos Charalambous, Paul Davis, Jessica C. E. Irving, Taichi Kawamura, Martin Knapmeyer, Ross Maguire, Angela G. Marusiak, Mark P. Panning, Clément Perrin, Ana-Catalina Plesa, Attilio Rivoldini, Cédric Schmelzbach, Géraldine Zenhäusern, Éric Beucler, John Clinton, Nikolaj Dahmen, Martin van Driel, Tamara Gudkova, Anna Horleston, Matthieu Plasman, W. Thomas Pike, Suzanne E. Smrekar

Correspondence to: $\underline{\text { simon.staehler@erdw.ethz.ch }}$

This PDF file includes:

Overview

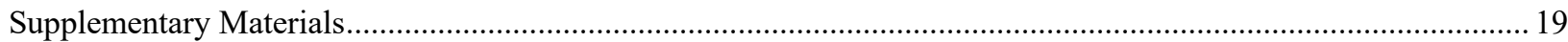

Table S0. List of candidate marsquakes for ScS detection ...........................................................................2 21

1. Method A: Polarization frequency-dependent filtering of marsquake waveforms ............................................. 22

Table S1-1. Overview over polarization/frequency-based filtering methods employed in this study .....................24

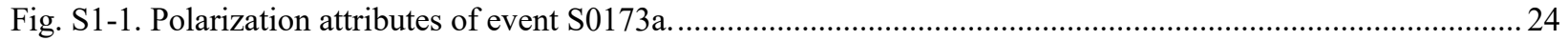

Fig. S1-2: Histogram for the distribution of measured ScS azimuth values for S0173a ...................................25

Fig. S1-3. Example of polarization filtering using the S-transform on event S0173a ......................................25

Fig. S1-4. Polarization-filtered transverse component seismograms for seven major marsquakes......................... 26

2. Method B: Horizontal and vertical rectilinear motion based on frequency-dependent polarization analysis ......... 27

Table S2-1. Polarization attributes and combined polarization metrics used in this analysis. ...............................29

Fig. S2-1. Frequency dependent polarization analysis with synthetic waveforms. ...........................................30

Fig. S2-2. Frequency dependent polarization analysis with real event waveforms on Earth. ...............................31

Fig. S2-3. Frequency dependent polarization analysis of event S0173a on Mars. ...........................................32

Fig. S2-4. Frequency dependent polarization analysis with six LF marsquakes. ................................................... 33

Table S2-2. Estimated phase arrivals for each event from frequency-dependent polarization analysis.................. 34

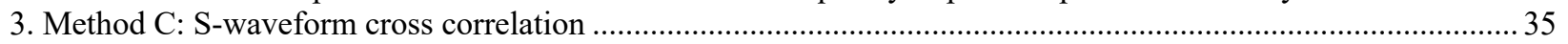

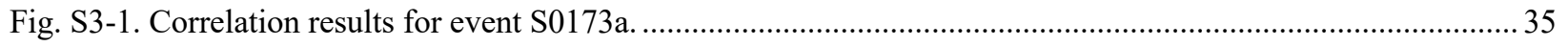

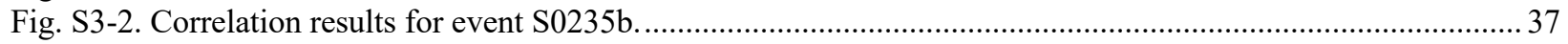

4. Method D: Event backazimuth estimation and detection of ScSH from correlation ........................................3.

Fig. S4-1. Example of phase identification for event S0235b. ...................................................................... 39

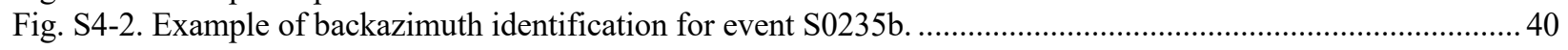

Fig. S4-3. Example of ScS identification for event S0235b. ...................................................................... 41 
Table S4-1. ScS picks from joint SH-correlation and backazimuth analysis.................................................... 41

5. Method E: Vespagram analysis of polarization filtered waveforms ............................................................42

Table S5-1. Summary of ScS travel-time picks, event backazimuths and SNR from Vespagram analysis.............. 43

Fig. S5-1. Polarization filtered waveforms as input for the vespagram analysis. ..............................................44

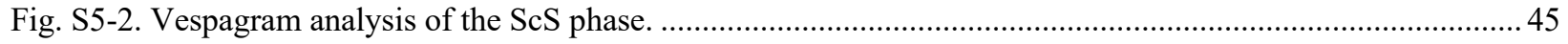

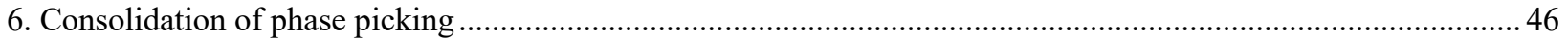

Fig. S6-1. Amplitudes and spectra of $3 \mathrm{ScS}$ picks (transverse component), compared to the teleseismic S-wave of a

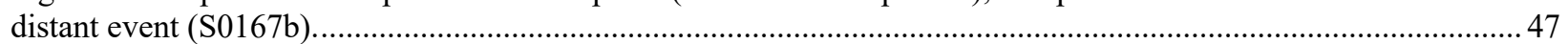

Fig. S6-2. Ray-theoretical amplitudes of core-reflected phases for solid and fluid outer core ................................. 48

Fig. S6-3. Filter bank for S0235b - S-phase ............................................................................................ 49

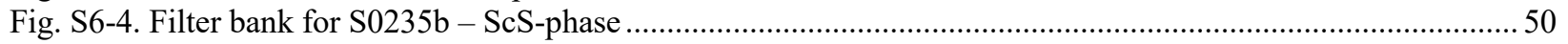

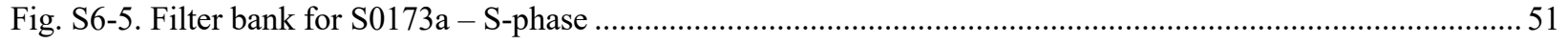

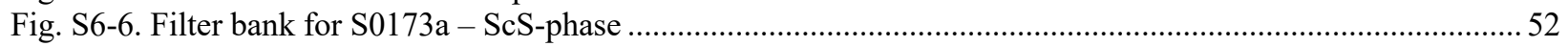

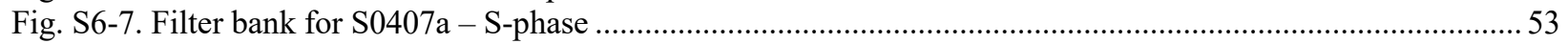

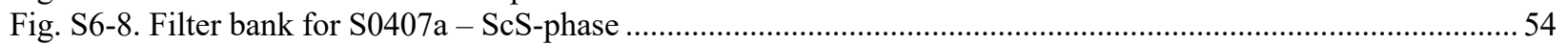

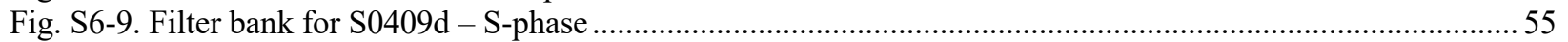

Fig. S6-10. Filter bank for S0409d - ScS-phase ......................................................................................... 56

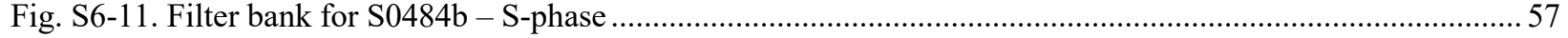

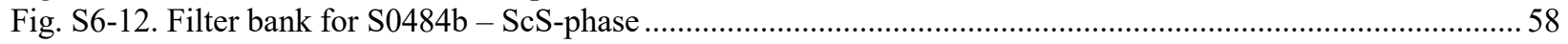

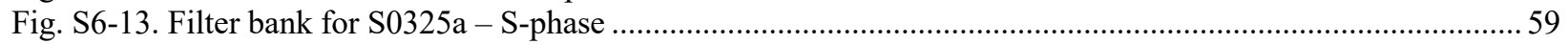

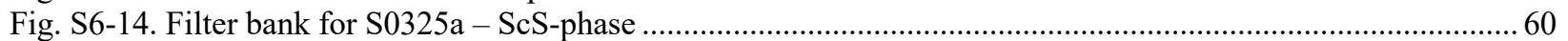

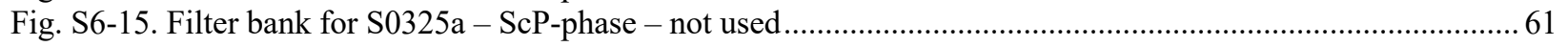

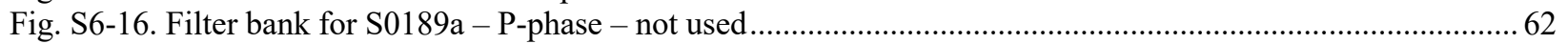

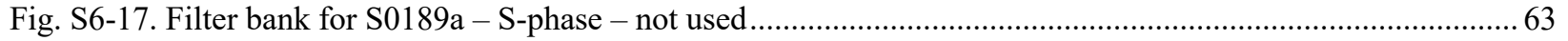

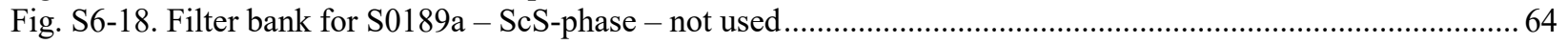

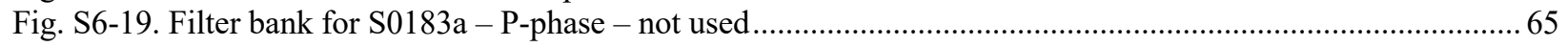

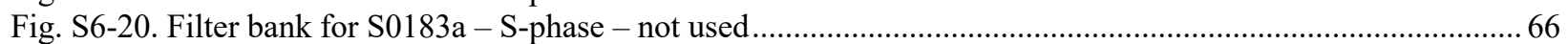

Fig. S6-21. Filter bank for S0183a - ScS-phase - not used ........................................................................67

7. Significance of observed phases with respect to seismic noise .......................................................... 71

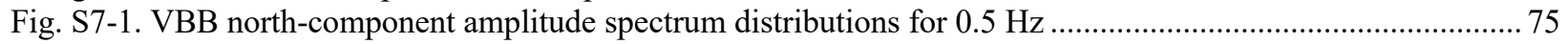

Fig. S7-2. Wind speed and seismic amplitude spectra comparison for S0173a ScS. ..................................... 76

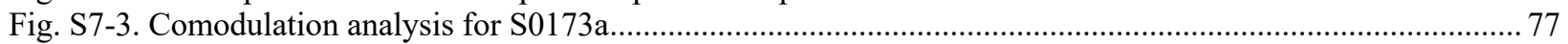

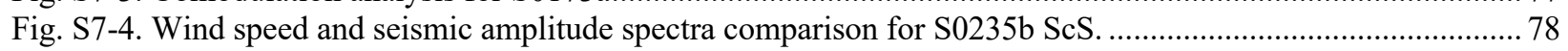

Fig. S7-5. Wind speed and seismic amplitude spectra comparison for S0325a ScS. .........................................79

Fig. S7-6. Wind speed and seismic amplitude spectra comparison for S0407a ScS. ...................................... 80

Fig. S7-7. Wind speed and seismic amplitude spectra comparison for S0409d ScS. ......................................... 81

Fig. S7-8. Wind speed and seismic amplitude spectra comparison for S0484b ScS. ....................................... 82

Fig. S7-9. The seismic amplitude spectra comparison for and the mean SNR values obtained at S0784a ScS........ 83

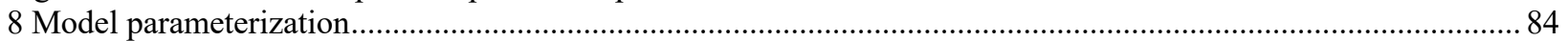

Table S8-1. Seismic model parameterization and prior model parameter information. ............................................85

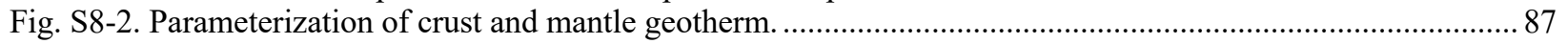

Table S8-2. Geophysical model parameterization and prior model parameter information. ................................... 88

Table S8-3. Geodynamical model parameterization and prior model parameter information................................ 90

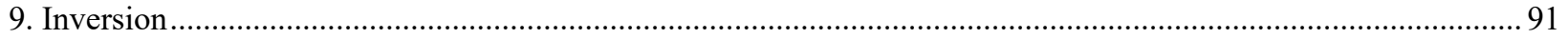

Fig. S9-1. A priori probability density functions (pdfs) of the 1-D (a) Vs and (b) Vp profiles..............................93

Fig. S9-2. A posteriori probability density functions (pdfs) of the 1-D mantle profiles........................................94

Fig. S9-3. Posterior distribution of models in the geodynamic inversion........................................................ 95

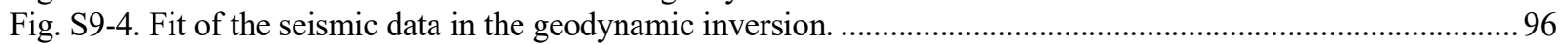

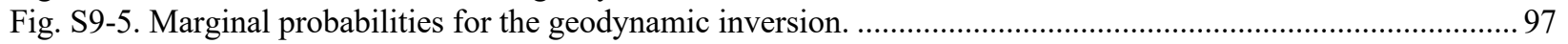

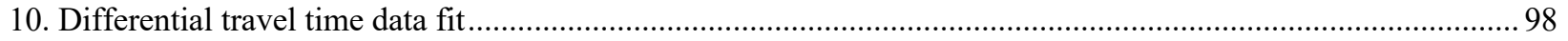

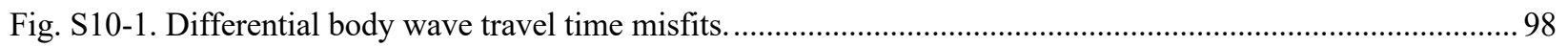

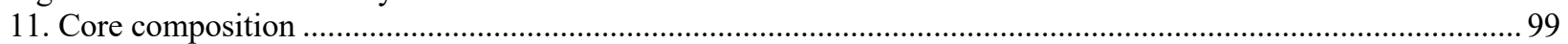

Fig. S11-1. Mean core density as a function of core sulfur fraction. .......................................................... 100 


\section{Overview}

The supplementary material is divided into four main parts:

1. Sections 1-5, which present several complementary efforts to detect and characterize $\mathrm{ScS}$ arrivals;

2. Sections 6-7, which combines the $\mathrm{ScS}$ analyses and characterizes the corresponding environmental noise levels;

3. Sections 8-10, which describe the inversion for structural models;

4. Section 11, which describes the implications of the structural models for Martian core composition.

For detection of $\mathrm{ScS}$, a total of 11 marsquakes were examined: all are events characterized as quality A or B by the marsquake service (MQS) by February 2021 (see table S0). The definitions of marsquake quality require that Quality A and B events have a clear identification of a P and an S arrival, which allows epicentral distance to be constrained. Quality A events also have a clearly identified backazimuth based on the P-wave polarization. In MQS operational practice, this polarization is determined from linear fitting of the P-wave motion (Böse et al. 2016). This method is insufficient for determining the polarization of low-SNR secondary phases, so several, more advanced methods have been used to qualify these.

For the purpose of validation, different groups led by researchers at ETH Zurich (ETH), Institut de Physique du Globe de Paris (IPGP), University of Maryland (UMd), Geosciences Barcelona (CSIC), and Institut Supérieur de l'Aéronautique et de l'Espace (ISAE), each determined their own ScS picks independently for different marsquake events using different methods. Each of the methods, which we refer to as Methods A through E, are described below. While not all groups provide picks for the same set of events, in general we find that travel time picks based on different methodologies are in good agreement, which provides confidence in our results and allows for an assessment of the travel time uncertainty of ScS. Section S6 presents a review of all individual picks that lead to the picks used in the inversion and listed in table 1.

\begin{tabular}{|l|l|l|l|l|l|l|}
\hline Name & Quality & Origin time & Distance & SNR & $\mathrm{M}_{w}$ & ScS detected \\
\hline S0784a & B & $2021-02-09 T 12: 11: 43$ & $33.6 \pm 3.6$ & 21 & 3.2 & weak, just for confirmation \\
\hline S0484b & B & $2020-04-07 T 08: 48: 03$ & $30.9 \pm 5.9$ & 4 & 2.8 & weak \\
\hline S0409d & B & $2020-01-21 T 11: 27: 43$ & $30.4 \pm 5.9$ & 26 & 3.1 & weak \\
\hline S0407a & B & $2020-01-19 T 09: 53: 42$ & $28.6 \pm 2.1$ & 3.9 & 3.0 & weak \\
\hline S0325a & B & $2019-10-26 \mathrm{~T} 06: 54: 00$ & $38.5 \pm 6.0$ & 9.8 & 3.7 & weak \\
\hline S0290b & B & $2019-09-21 \mathrm{~T} 03: 15: 46$ & $29.5 \pm 9.5$ & 1.4 & 3.4 & no \\
\hline S0235b & A & $2019-07-26 \mathrm{~T} 12: 15: 36$ & $27.9 \pm 1.5$ & 290 & 3.5 & clear \\
\hline S0189a & B & $2019-06-09 T 05: 35: 50$ & $32.7 \pm 8.6$ & 5 & 2.8 & no \\
\hline S0185a & B & $2019-06-05 T 02: 07: 01$ & $58.4 \pm 21.4$ & 8 & 3.0 & no \\
\hline S0183a & B & $2019-06-03 T 02: 21: 56$ & $45.8 \pm 17.6$ & 3.1 & 3.1 & Unclear S-phase \\
\hline S0173a & A & $2019-05-23 T 02: 19: 17$ & $29.3 \pm 1.6$ & 93 & 3.6 & clear \\
\hline
\end{tabular}

\section{Table S0. List of candidate marsquakes for ScS detection}

taken from the MQS catalog v6 (30). The origin time and distance are rapid solutions that have been determined from a suite of 2500 pre-mission models (as described in (89)). 


\section{Method A: Polarization frequency-dependent filtering of marsquake waveforms}

Here, we provide details on the extraction of potential $\mathrm{ScS}$ core phases using a dedicated polarization filter. The polarization of a seismic signal characterizes its particle motion and permits the determination of wave type and the azimuth and inclination angles of the incoming wave. Our polarization approach is based on the eigen-analysis of the time-frequency dependent spectral matrix which is built from the three component (3-C) seismic recordings (36). The timefrequency representation of the spectral matrix is obtained through the S-transform of the time domain recordings (90). This approach relies on Gaussian-shaped windows which are scaled inversely with frequency, i.e., with narrower windows at higher frequencies and wider windows at lower frequencies. Further, the Gaussian shape permits an optimal time-frequency resolution. Another advantage of the S-transform is that it is fully reversible, which allows us to bring the data back to the time domain after filtering.

The polarization of a pure-state seismic wave in three dimensions can be described by the polarization ellipse, representing the 3D particle trajectory of a seismic wave at a single station (91, 92). Figure S1-1 shows an example of the polarization of event S0173a, using the attributes defined in (93), which are repeated in the following: The eccentricity of the ellipse is defined by the ratio of the lengths of its minor and major semi-axes and can be used to distinguish rectilinearly polarized waves (such as P-, and S-phases) from elliptically polarized Rayleigh waves and noise. For rectilinearly polarized waves, the direction of the major semi-axis provides information on the propagation direction (azimuth and inclination) of the wave, which lies parallel to the axis for P-waves and orthogonal to it for S-waves. As shown in fig S1-2, the strongly polarized signal 511 seconds after P for S0173a has an azimuth which is consistent with $\mathrm{ScS}$. To improve the data of the weaker event, we will further exploit these polarization attributes to filter the data, with the goal to enhance S-wave phases at close-to-vertical incidence (as expected for the $\mathrm{ScS}$ core phase). We further employ a measure of polarization quality which we call the degree of polarization $(D O P,(36))$.

In an ideal, noise-free scenario, the spectral matrix would only have one non-zero eigenvalue if a single pure-state seismic arrival is present in the analysis window. The real and imaginary parts of the associated complex eigenvector would then describe the direction and length of the major and minor semi-axis of the polarization ellipse. In practice, the polarization will always be affected by noise and interfering wave arrivals in the analysis window (e.g., due to multipathing or scattering) causing a complex 3D particle trajectory that deviates from an ellipse. We rely on a measure called the degree of polarization $(D O P)$ in order to avoid interpreting parts of the signal that are only weakly polarized. This quantity assumes that an arriving seismic phase has an arbitrary, but stable polarization throughout the course of the signal and is measured within a frequency-dependent moving window. The $D O P$ of the wave can be computed from the three eigenvalues $\lambda_{1}, \lambda_{2}$, and $\lambda_{3}$ of the spectral matrix via (92):

$$
\operatorname{DOP}^{2}=\sum_{j, k=1}^{3} \frac{\left(\lambda_{j}-\lambda_{k}\right)^{2}}{4\left(\sum_{j, k=1}^{3}\left(\lambda_{j}\right)^{2}\right)}
$$


If a polarized wave arrival is present in the analysis time-frequency window, one eigenvalue will be significantly larger than the others and $D O P$ will tend to 1 . If there is no polarized signal in the analysis window, $D O P$ will tend to 0 . In order to suppress weakly polarized signals in the marsquake data, we rejected all parts of the signal with a degree of polarization $D O P<0.6$ by setting the corresponding part of the S-transformed data to zero. Note that a different definition of the degree of polarization has been found by (36), which is not amplitude-biased and has been used here (Figure S1-1, S1-2). For the remaining signal, we estimated the ellipticity and incidence angle from the length and direction of the real and imaginary parts of the principal eigenvector associated with the largest eigenvalue of the spectral matrix, which correspond to the major and minor semi-axis of the polarization ellipse. The ellipticity is 1 for a circularly polarized signal and 0 for a perfectly rectilinearly polarized signal. ScS core phases are expected to be linearly polarized, so we suppressed all signal with an ellipticity larger than 0.4 in order to reduce unwanted noise and suppress surface waves.

The inclination of the 3D particle motion (measured from the horizontal) can be estimated from the real part of the components of the principal eigenvector (i.e., the major semi-axis) $e_{i}$ via

$$
\tan \theta=\frac{\mathfrak{R}\left(e_{Z}\right)}{\sqrt{\mathfrak{R}\left(e_{N}\right)^{2}+\mathfrak{R}\left(e_{E}\right)^{2}}}
$$

Since an S-wave at close to vertical incidence (such as an ScS core phase) will cause the ground to vibrate predominantly in the horizontal direction, we rejected all signal with an inclination angle larger than 30 degrees from the horizontal. After filtering, the data is converted back to the time domain via the inverse S-transform. Figure S1-3 shows an example of applying the described polarization filter to the transverse component data of marsquake event S0173a. Before polarization filtering, there are no clear arrivals following the $\mathrm{S}$ phase (Fig. S1-3a). However, after filtering, a signal with polarization attributes consistent with $\mathrm{ScS}$ appears at $\sim 510$ $\mathrm{s}$ after the P-wave (Fig S1-3e).

Figure S1-3 shows polarization filtered waveforms for seven major marsquakes with an additional bandpass filter between 0.3 and $0.6 \mathrm{~Hz}$. An excess of horizontally and rectilinearly polarized energy at the expected arrival time of the ScS arrival about 500 seconds after the Pwave arrival can be observed for multiple events. The seismograms in Fig. S1-4 are aligned on the identified $\mathrm{ScS}$ arrival times listed in Table 1 of the main paper. An envelope stack of the filtered waveforms shows a distinct increase in stacked energy at the suspected $\mathrm{ScS}$ arrival time (Fig. S1-4b and Fig. 1 of the Main Text).

This method was applied independently by researchers at ETH Zürich (2 teams) and IPGP Paris/GEO3BCN-CSIC, resulting in 3 sets of picks (A1, A2, A3, see table S1-1.). The latter method is described in details in (93) where it was used for analyzing Mars noise data. For the values, see Section S6. 


\begin{tabular}{|l|l|l|l|}
\hline Method & Transformation & DOP definition & Events \\
\hline A1 & S-transform (90) & Samson (92) & $\begin{array}{l}\text { S0173a, S0235b, S0325a, S0407a, } \\
\text { S0409d, S0484b, S0784a }\end{array}$ \\
\hline A2 & S-transform (90) & Schimmel/Gallart (36) & S0173a, S0235b \\
\hline A3 & $\begin{array}{l}\text { Continuous Wavelet } \\
\text { transform (84) }\end{array}$ & Samson (92) & $\begin{array}{l}\text { S0173a, S0235b, S0325a, S0407a, } \\
\text { S0409d, S0484b }\end{array}$ \\
\hline
\end{tabular}

Table S1-1. Overview over polarization/frequency-based filtering methods employed in this study

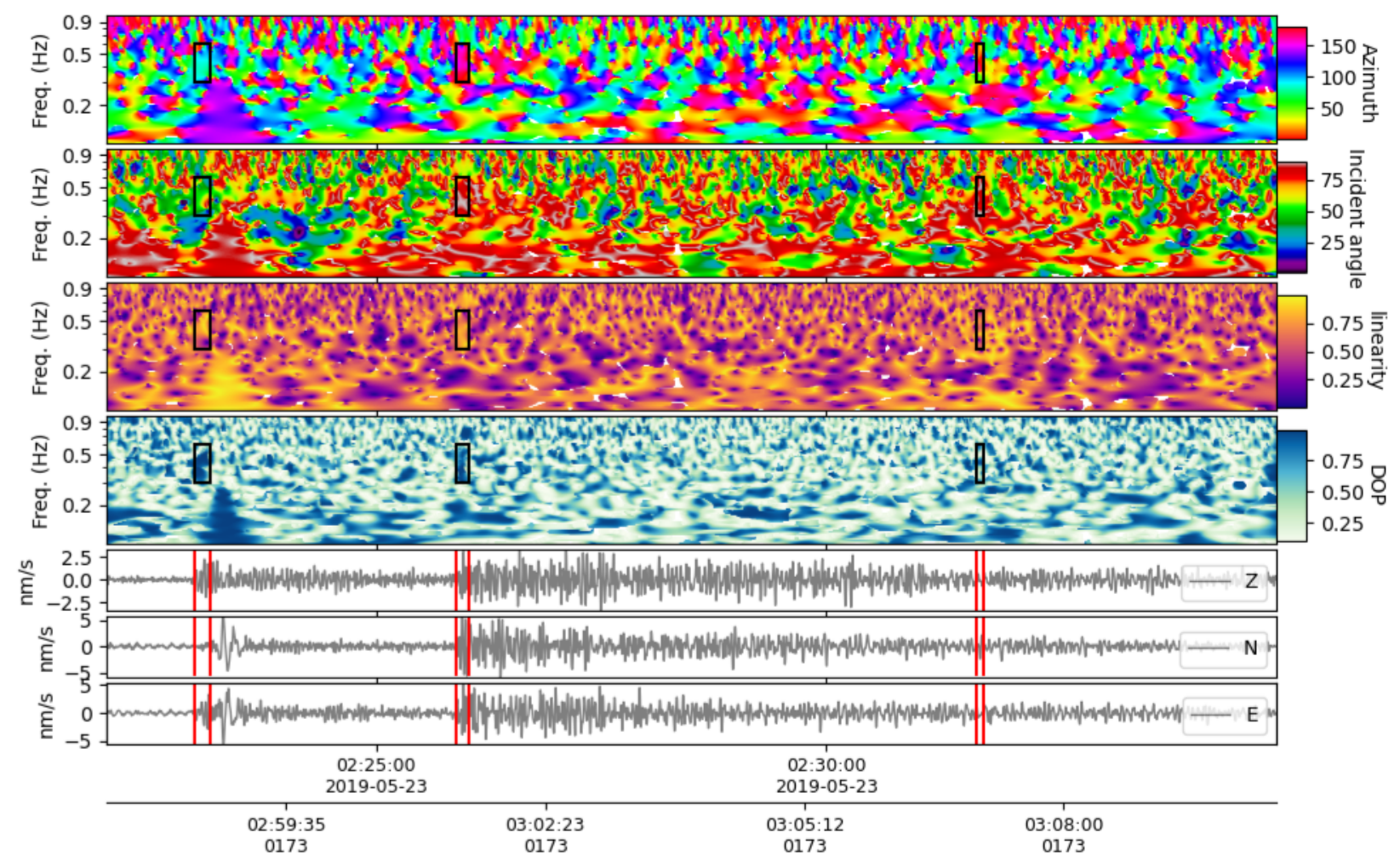

Fig. S1-1. Polarization attributes of event S0173a.

The subplots show the result of polarization analysis using the S-transform (90) and the degree of polarization (DOP), as defined in (36) (from top to bottom, see (93) for details): azimuth of the major axis of the polarization ellipse, incident angle of the major axis (from vertical), linearity, defined as 1 - ellipticity, DOP, and a 3 component seismogram. The DOP subplot shows increased polarization between 0.3 and $0.8 \mathrm{~Hz}$ in the P-wave (left box), the S-wave (middle box) and a third arrival 511 seconds after P (right box). 


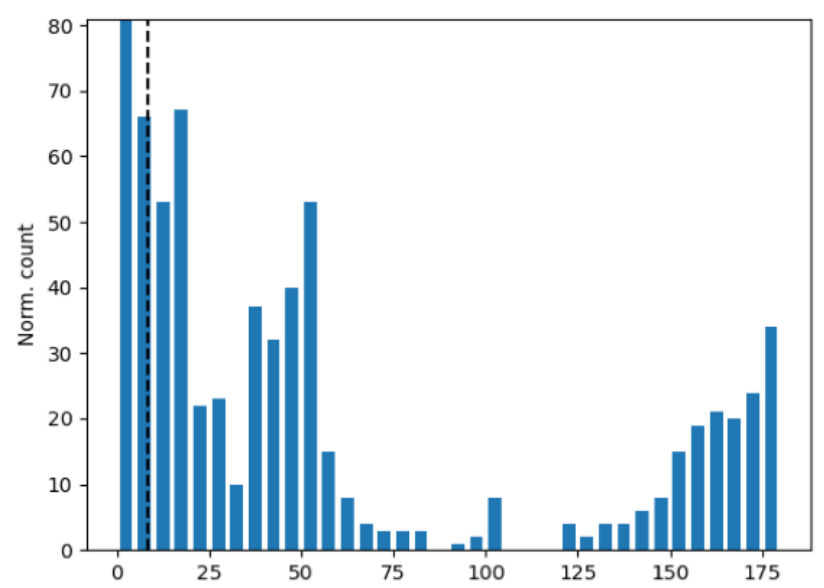

Fig. S1-2: Histogram for the distribution of measured ScS azimuth values for S0173a The value has been measured within the black box of fig S1-1. Since the backazimuth of the event has been determined to $91^{\circ}(30)$, the expected $\mathrm{ScS}-\mathrm{H}$ azimuth is $1 \mathrm{deg}$.
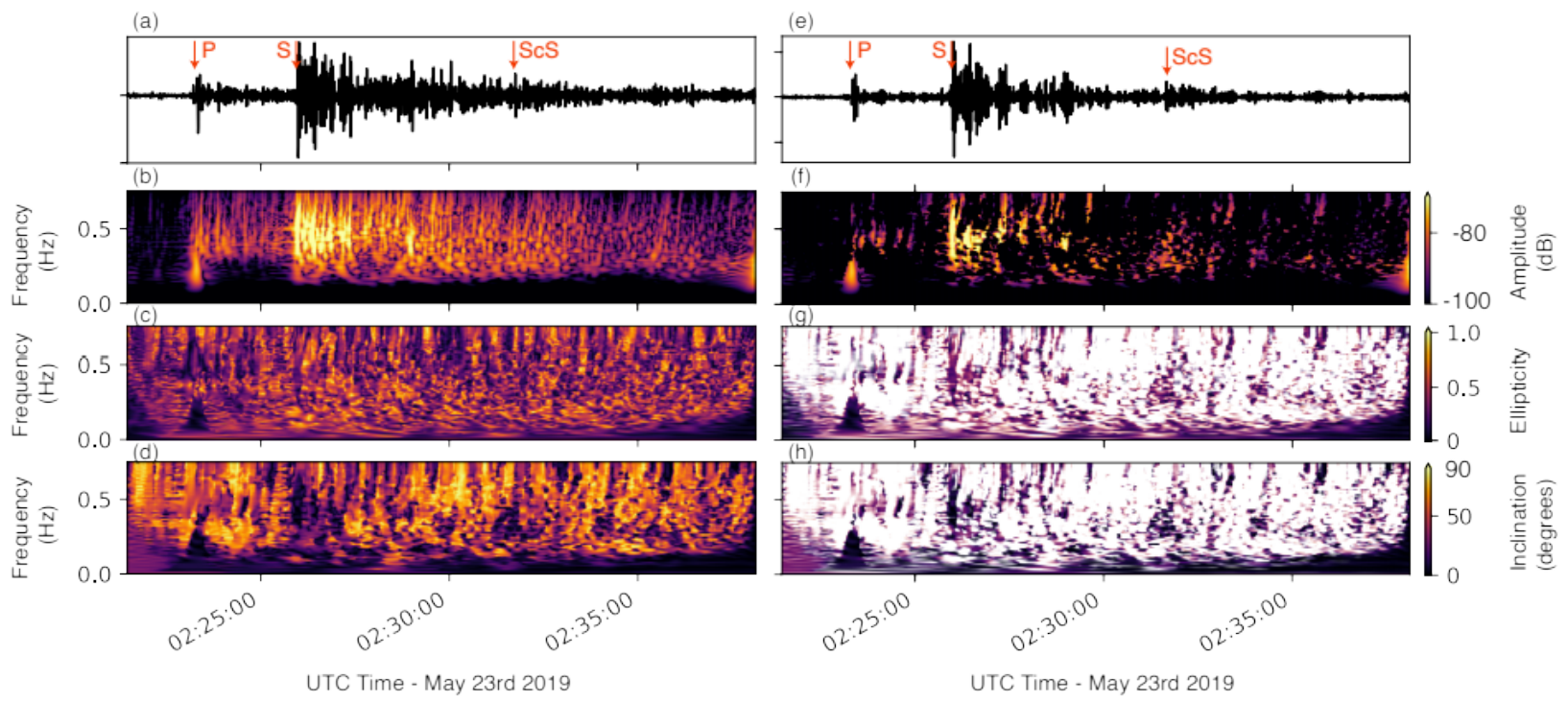

\section{Fig. S1-3. Example of polarization filtering using the S-transform on event S0173a}

The polarization attributes shown in fig. S1-1 are used to filter the spectrogram and seismograms. The input data is shown on the left, polarization-filtered to enhance S-waves (low inclination, high linearity) on the right, and some key phases are marked in red. (a) Transverse component seismogram. (b) S-transform of data shown in (a). (c) Time- and frequencydependent ellipticity obtained from polarization analysis. (d) Inclination angle (in degrees) of the major axis of particle motion. (e) Polarization-filtered transverse component seismogram. (f) Stransform of data shown in (e). (g) Time- and frequency-dependent ellipticity (only signal with ellipticity $<0.4$ is retained). (h) Inclination angles (only horizontally polarized signals with an inclination angle $<30^{\circ}$ are retained). The strong, horizontally polarized signal 10 seconds after the P-wave arrival corresponds to a data glitch (39). 
(a)

Polarization-filtered transverse component seismograms (black) and envelopes (red)
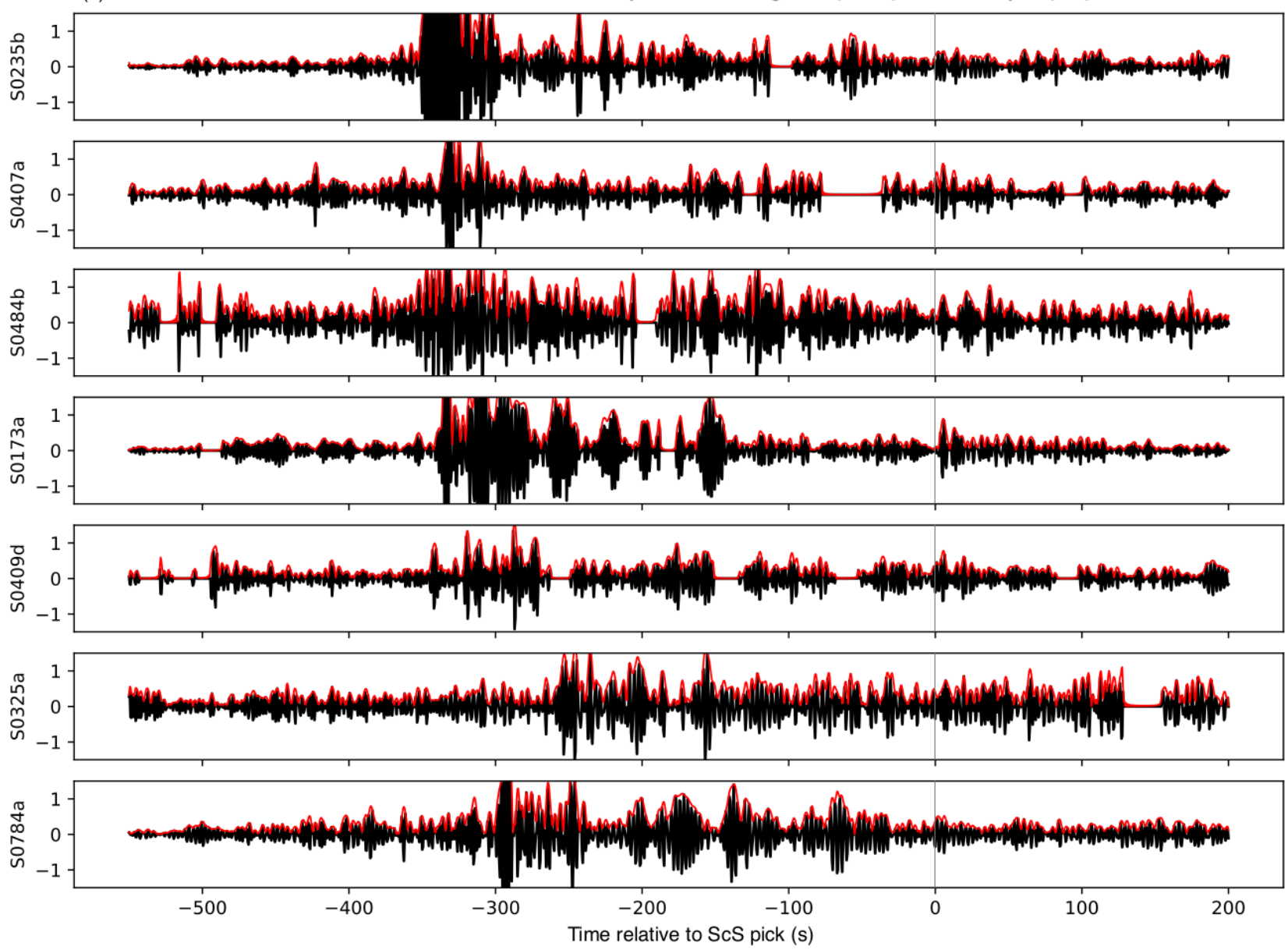

Fig. S1-4. Polarization-filtered transverse component seismograms for seven major marsquakes.

(a) The data is additionally filtered with a bandpass filter $(0.3-0.6 \mathrm{~Hz})$. All events show an increase in transversely and rectilinearly polarized energy about 500 seconds after the P-wave arrival. The data is aligned on picks of the proposed $\mathrm{ScS}$ arrivals. Known data glitches are manually set to zero (e.g., 90-50 seconds before ScS on S0407a). Red lines show the envelope of the waveforms. The stack of the envelopes for the first six events is shown in Fig 1. of the main paper as well. Event S0784a serves as an independent control. 


\section{Method B: Horizontal and vertical rectilinear motion based on frequency- dependent polarization analysis}

In the analysis described in this section, we conduct frequency-dependent polarization analysis (FDPA) on waveforms recorded by InSight SEIS VBB to identify the arrival of bodywave phases. We demonstrate the efficacy of our method on synthetic and Earth signals before applying it to the seven most suitable LF marsquakes. We can find arrivals which meet the characteristics expected of core-reflected $S c S$ for five of the seven marsquakes. We also find a signal arriving before the $S c S$ arrival; we are not certain of this phase's identity. $P c P$ and $S c P / P c S$ are not observed in the available data using this method.

We start by computing the S-transform of three component event waveforms and compute a $3 \times 3$ cross-spectral covariance matrix in $90 \%$ overlapping time windows whose duration varies inversely with frequency. The relative sizes of the eigenvalues of this covariance matrix are related to the degree of polarization $(D O P)$ of the particle motion, while the complex-valued components of the eigenvectors describe the particle motion ellipsoid in each time-frequency window (e.g., (94)). Here, we focus on a subset of polarization attributes (summarized in Table. $\mathrm{S} 2-1$ ). When one eigenvalue is much larger than the others, the corresponding $D O P$ is high, and interpreting the particle-motion ellipsoid corresponding to the dominant eigenvector is warranted. If the $D O P$ is high, and the complex-valued coefficients of the dominant eigenvector are in phase $\left(\cos \left(\varphi_{H H}\right)=1, \cos \left(\varphi_{H V}\right)=1\right)$ the motion is rectilinear; in this case, we can effectively describe the orientation of the particle motion with two angles, the inclination $\theta_{V}$ and azimuth $\theta_{H}$. For weakly polarized seismic energy (i.e., when the eigenvector coefficients are out of phase), the polarization attributes are highly variable with time and frequency and should not be interpreted.

We seek arrivals compatible with $S c S$ and $P c S$ that are strongly linearly-polarized in the horizontal direction, and for arrivals compatible with $P c P$ and $S c P$ that are strongly linearlypolarized in the vertical direction. Therefore, we combine polarization attributes into a pair of metrics, emphasizing horizontally rectilinear motion (HRM) and vertically rectilinear motion (VRM), and search for candidate arrivals whose average HRM or VRM across different frequencies are high. We make no requirements of the phase's backazimuth as these estimates have a high uncertainty even for the direct $P$ and $S$ phases. We validate our HRM and VRM measurements on synthetic signals embedded in background random white noise with amplitude plausible for Mars. In Fig. S2-1, we show that while arbitrarily polarized signals are subtle and difficult to identify in individual waveforms (Fig. S2-1A) they become readily apparent in the HRM and VRM metrics. In contrast, elliptically polarized synthetic signals, representative of Rayleigh waves, are characterized by very low HRM and VRM values (Fig. S1B-C). This validates the approach of using combined polarization attributes to identify arrivals of linearly polarized energy. In Fig. S2-2, we apply the method to earthquake waveforms recorded from a $M_{W} 6.4$ event (CMT code 202011302254B) and find that the HRM and VRM metrics identify arrivals of body wave phases, including $P c P$ and $S c S$. We note that for this station, the direct $S$ arrival has a low $D O P$, most likely due to shear-wave splitting, so that it is associated with relatively low HRM and VRM values (Fig. S2-2B and S2-2D).

We compute the HRM and VRM measurements for seven high-quality LF Mars events: S0173a (highlighted in Fig. S2-3), S0183a, S0235b, S0325a, S0407a, S409d, and S0484b, which have been extensively analyzed by various members of the InSight team (32). The SEIS-VBB 3- 
component waveforms are rotated to the ZNE coordinate frame and are bandpass filtered between $0.2-0.8 \mathrm{~Hz}$ to focus on the LF energy of each marsquake (Fig. S2-3A). The $1 \mathrm{~Hz}$ tick noise (electronic disturbance due to crosstalk between the seismometer and the temperature sensor acquiring data at $1 \mathrm{sps}$ ) should not be a concern in our frequency range of interest. We replace known glitches (39) by timeseries generated by randomizing phases information from the background, glitch-absent, pre-event noise while retaining its amplitude information. We use reference $P$ - and $S$-arrival times from the MQS and extract an analysis window starting $50 \mathrm{~s}$ before to $900 \mathrm{~s}$ after the $P$-arrivals. For the core phases, we use several existing reference models (DWThotCrust1, DWThotCrust2 and DWTcoldCrust1 (16); MAAK (4) as well as more recent ones (32)) to predict travel times across a range of different core radii $(1580-1850 \mathrm{~km})$.

Our ScS candidate phase picks are determined as follows: (1) We start by identifying a clear, strongly linearly-polarized $P$ - and $S$-arrival on the averaged VRM and HRM, respectively. For each event, if both of our polarization metrics are inconsistent to the MQS travel times for $P$ and $S$-arrivals, we discard that event. (2) Within the $S c S$ prediction time window, we identify peaks with a HRM maximum and simultaneous VRM minimum where (a) HRM/VRM $>1$ and (b) $\mathrm{HRM}_{\max }>\mathrm{HRM}_{\text {mean }}+\sigma_{\mathrm{HRM}}$ and (c) $\mathrm{VRM}_{\min }<\mathrm{VRM}_{\text {mean }}-\sigma \mathrm{V}_{\mathrm{RM}}$, where (b) and (c) are equivalent to the condition that the HRM maxima and VRM minima for the peak deviate by more than 1-sigma from the mean HRM and VRM in the $S c S$ prediction window (yellow bars, Fig. 4). (3) If two or more arrivals satisfy (1) and (2), we choose the arrival with the largest HRM/VRM. Based on our polarization analysis, we identify five candidate $S c S$ phases (black arrows, Fig. S4; Table S2-1). This corresponds to one for each event apart from S0325a and $\mathrm{S} 0409 \mathrm{~b}$. This indicates the seismic energy associated with the candidate phases is predominantly linearly-polarized in the horizontal direction, as expected for the steeply-incident $S c S$ phase.

Multiple HRM maxima are observed for S0407a, S0484b, and S0183a within the ScS prediction window, which could result from noise or from complexity in the core-mantle boundary region. Arrivals $16-76 \mathrm{~s}$ before the apparent $S c S$ are consistently observed for S0235b, S0407a, S0484b, and S0183a (red arrows, Fig. S4). Despite the uncertainties associated with event locations and depths (32), these arrivals are not likely to be compatible with the $S c S$ phase. Instead, these $S c S$ precursors warrant further modeling, and may be associated with a low velocity partially molten layer at the base of the mantle, the presence of which has been proposed for Mars (79). A similar structure has been reported on the Moon based on Apollo data (24), and large ultralow-velocity zones near the Earth's core produce $S c S$ pre- and post-cursors.

Interpretation of HRM and VRM metrics associated with $P c P$ is complicated by interference with the arrivals of $S$ and its multiples. We are not able to identify consistent arrivals for $S c P / P c S$. 


\begin{tabular}{|c|l|c|}
\hline Attribute & \multicolumn{1}{|c|}{ Attribute description } & Reference \\
\hline$\phi_{H H}$ & $\begin{array}{l}\text { Phase angle difference between the two horizontal components of motion, } \\
\text { restricted within }-180^{\circ} \text { to } 180^{\circ}\end{array}$ & $(94)$ \\
\hline$\phi_{H V}$ & $\begin{array}{l}\text { Phase angle difference between the vertical-horizontal components of the motion, } \\
\text { restricted within }-90^{\circ} \text { to } 90^{\circ}\end{array}$ & $(94)$ \\
\hline$\theta_{\mathrm{V}}$ & $\begin{array}{l}\text { Vertical angle of incidence of the particle motion, restricted within } 0^{\circ} \text { to } 90^{\circ} \\
\text { DOP }\end{array}$ & $\begin{array}{l}\text { Degree of polarization }(\mathrm{DOP}) \text { of the particle motion; } \\
D O P=\left(3 \times \text { trace }\left(C^{2}\right)-\text { trace }(C)^{2}\right) /\left(2 \times \text { trace }(C)^{2}\right) \\
\text { where } C \text { denotes a } 3 \times 3 \text { cross-spectral covariance matrix at each time window }(\text { see } \\
\text { supplement } 1) . D O P \text { is } 1.0 \text { when data are described by a single non-zero } \\
\text { eigenvalue and } 0 \text { when all eigenvalues are equal. This metric is rotationally } \\
\text { invariant. }\end{array}$ \\
\hline HRM & $\begin{array}{l}\text { Horizontal rectilinear motion } \\
(H R M)=\left|\cos \phi_{H V}\right| \times\left|\cos \phi_{H H}\right| \times \sin \phi_{V} \times D O P\end{array}$ & $(92,95)$ \\
\hline VRM & $\begin{array}{l}\text { Vertical rectilinear motion } \\
(V R M)=\left|\cos \phi_{H V}\right| \times\left|\cos \phi_{H H}\right| \times \cos \phi_{V} \times D O P\end{array}$ \\
\hline
\end{tabular}

\section{Table S2-1. Polarization attributes and combined polarization metrics used in this analysis.}

See (96) for detailed discussion on the use of four individual attributes: $\varphi_{\mathrm{HH}}, \varphi_{\mathrm{HV}}, \theta_{\mathrm{v}}$, and $D O P$. HRM and VRM are derived by properly weighting the upper four attributes. Note each cosine term in bold for HRM and VRM can effectively suppress elliptically polarized waves. For processing marsquake waveforms, the HRM and VRM metrics have been simplified by dropping the bolded cosine terms due to the absence of surface waves (e.g., Fig. S2-3 and 2-4). 

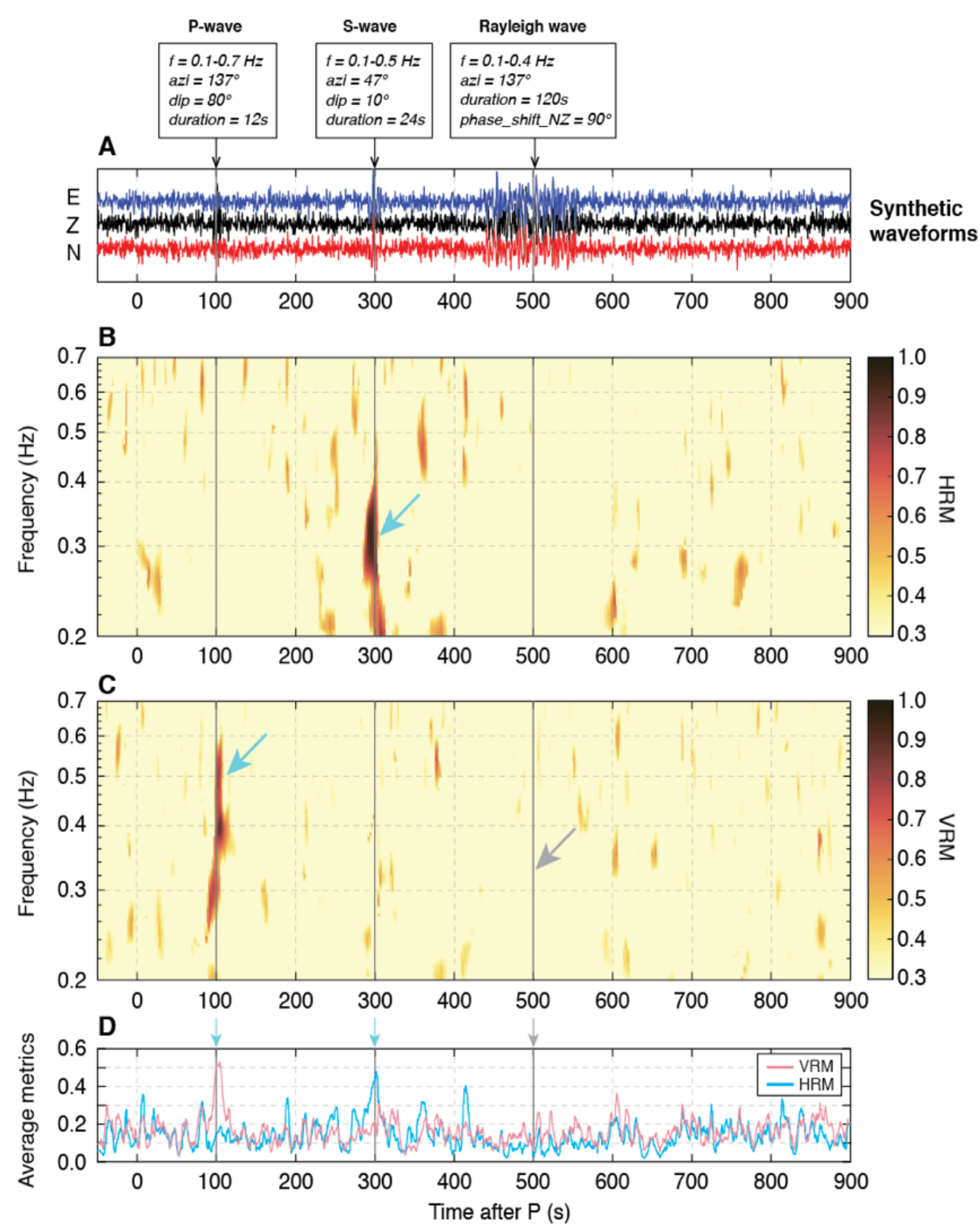

Fig. S2-1. Frequency dependent polarization analysis with synthetic waveforms.

(A) Three arbitrary signals with known azimuth, dip, phase angle, and frequency range are generated at $100 \mathrm{~s}, 300 \mathrm{~s}$, and $500 \mathrm{~s}$ and contaminated with white random noise with standard deviation of 0.2 , and with the noise amplitude being 0.7 times that of the Rayleigh wave. The first and second signals are linearly-polarized in the vertical and horizontal direction, respectively. In contrast, an elliptically polarized signal, representative of Rayleigh wave, is added to the synthetics. (B-C) Two combined polarization metrics (HRM and VRM) computed for synthetic data shown in S2-1A. (D) These metrics are averaged across $0.2-0.7 \mathrm{~Hz}$. Gray vertical lines and arrows indicate the correct timing and the central frequency of the three input signals. Note first two input signals at $100 \mathrm{~s}$ and $300 \mathrm{~s}$ are readily apparent while the last signal disappears in S2-1B-D because its particle motion is elliptically polarized. 

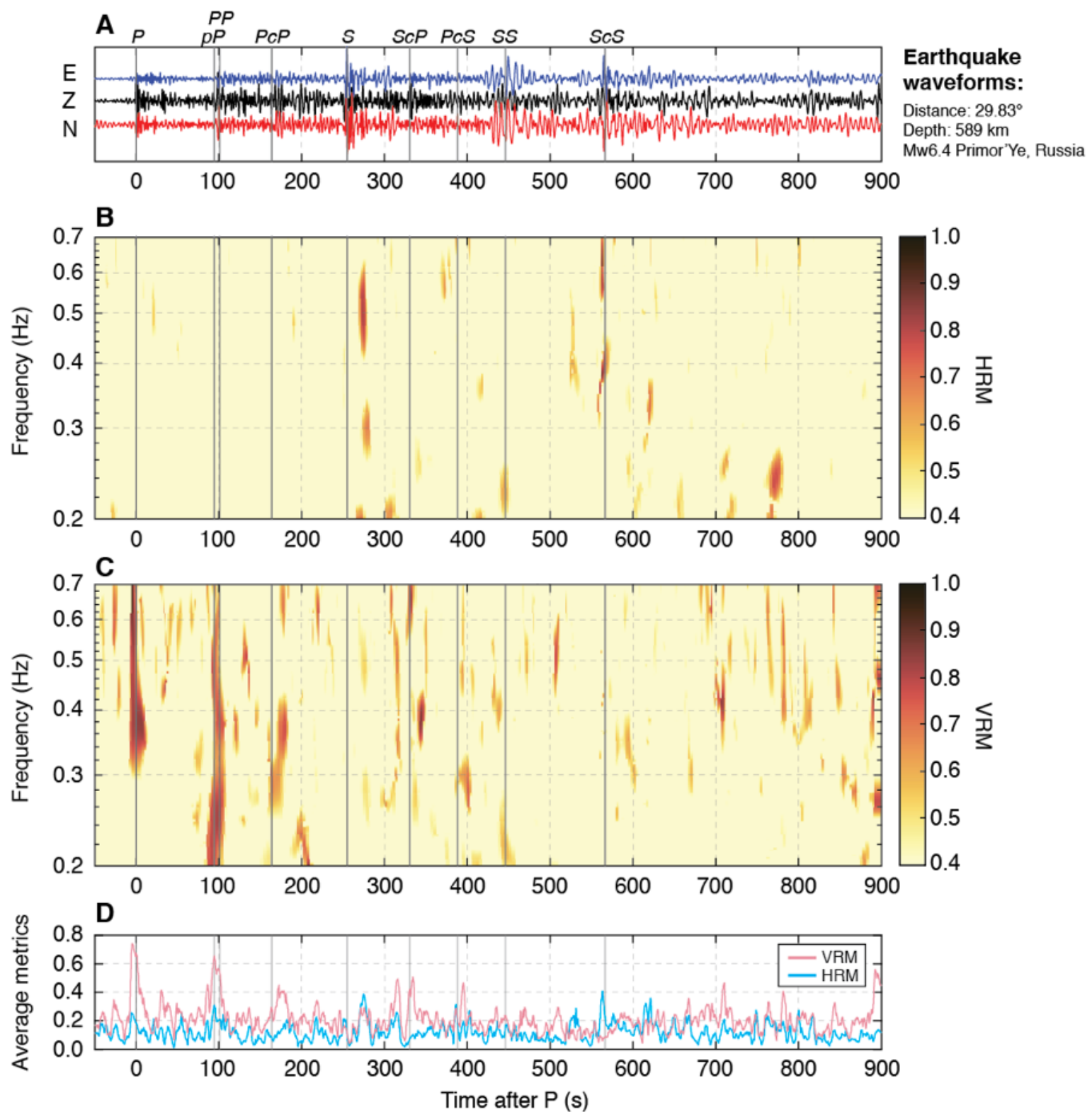

Fig. S2-2. Frequency dependent polarization analysis with real event waveforms on Earth.

(A) Mw6.4 Primor'Ye earthquake (2020-11-30UTC22:54:03) waveforms recorded by broadband station IC.ENH (BHE/BHN/BHZ), bandpass filtered between $0.1-0.9$ Hz. (B-C) Two combined polarization metrics (HRM and VRM) computed for the event data in S2-2A and (D) averaged across $0.2-0.7 \mathrm{~Hz}$. The HRM and VRM measurements show excellent agreement with predicted travel times of various body-wave phases (gray) including core phases especially for $P c P$ and $S c S$. Note relatively small HRM and VRM measurements around the direct $S$ are most likely due to shear-wave splitting. 


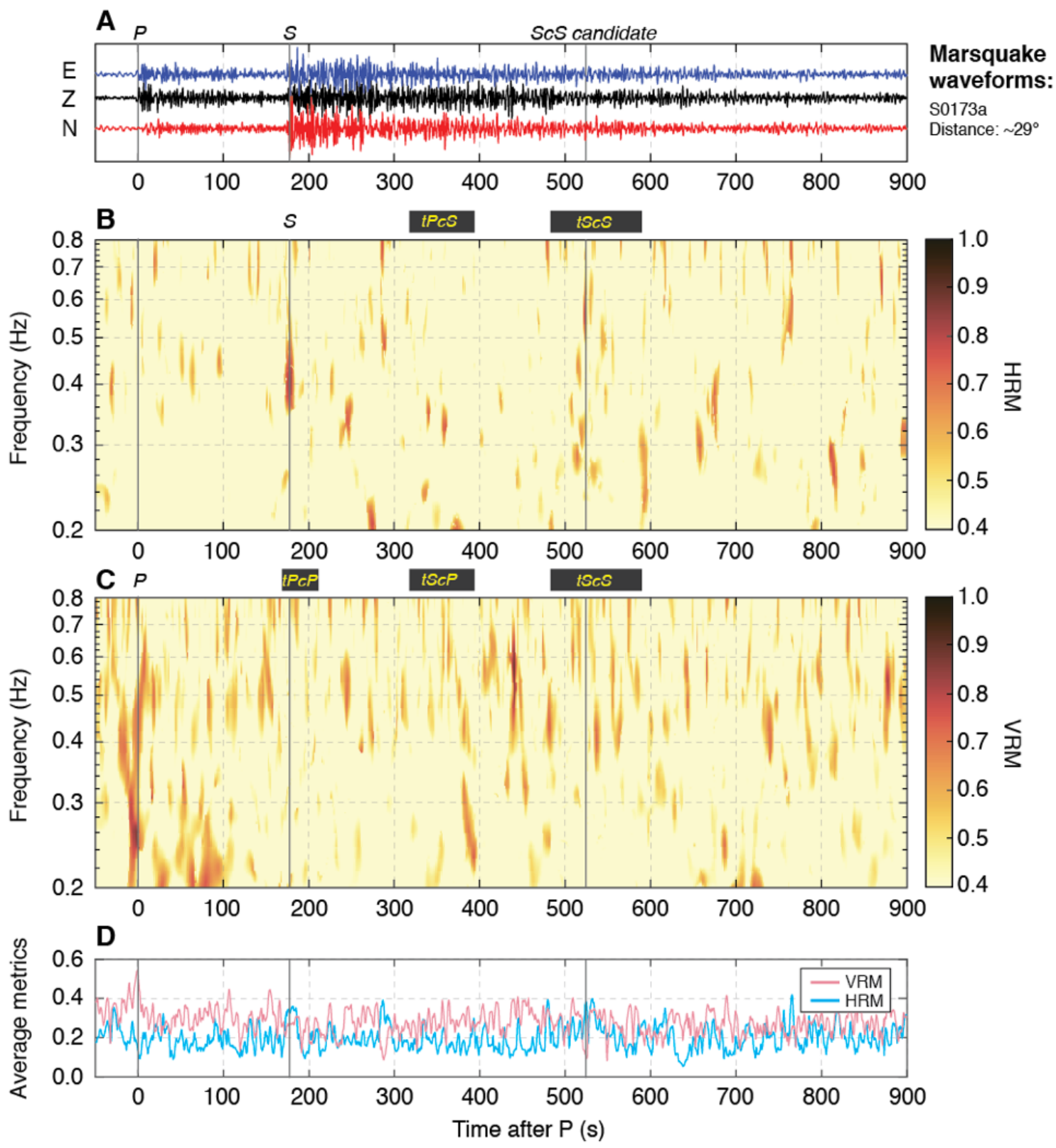

Fig. S2-3. Frequency dependent polarization analysis of event S0173a on Mars.

(A) S0173a (2019-05-23UTC02:19:16) waveforms recorded by SEIS VBB (BHE/BHN/BHZ) bandpass filtered between $0.2-0.8 \mathrm{~Hz}$. (B-C) Two combined polarization metrics (HRM and VRM) computed for the event data in S2-3A and (D) averaged across $0.3-0.8 \mathrm{~Hz}$. The HRM and VRM metrics have been simplified by dropping the bolded cosine terms in Table S2-1. Our polarization metrics show excellent agreement with P- and S-arrivals from the MQS. We identify a candidate $S c S$ phase $525 \mathrm{~s}$ after the $\mathrm{P}$ wave based on our phase picking procedure described in the text. 

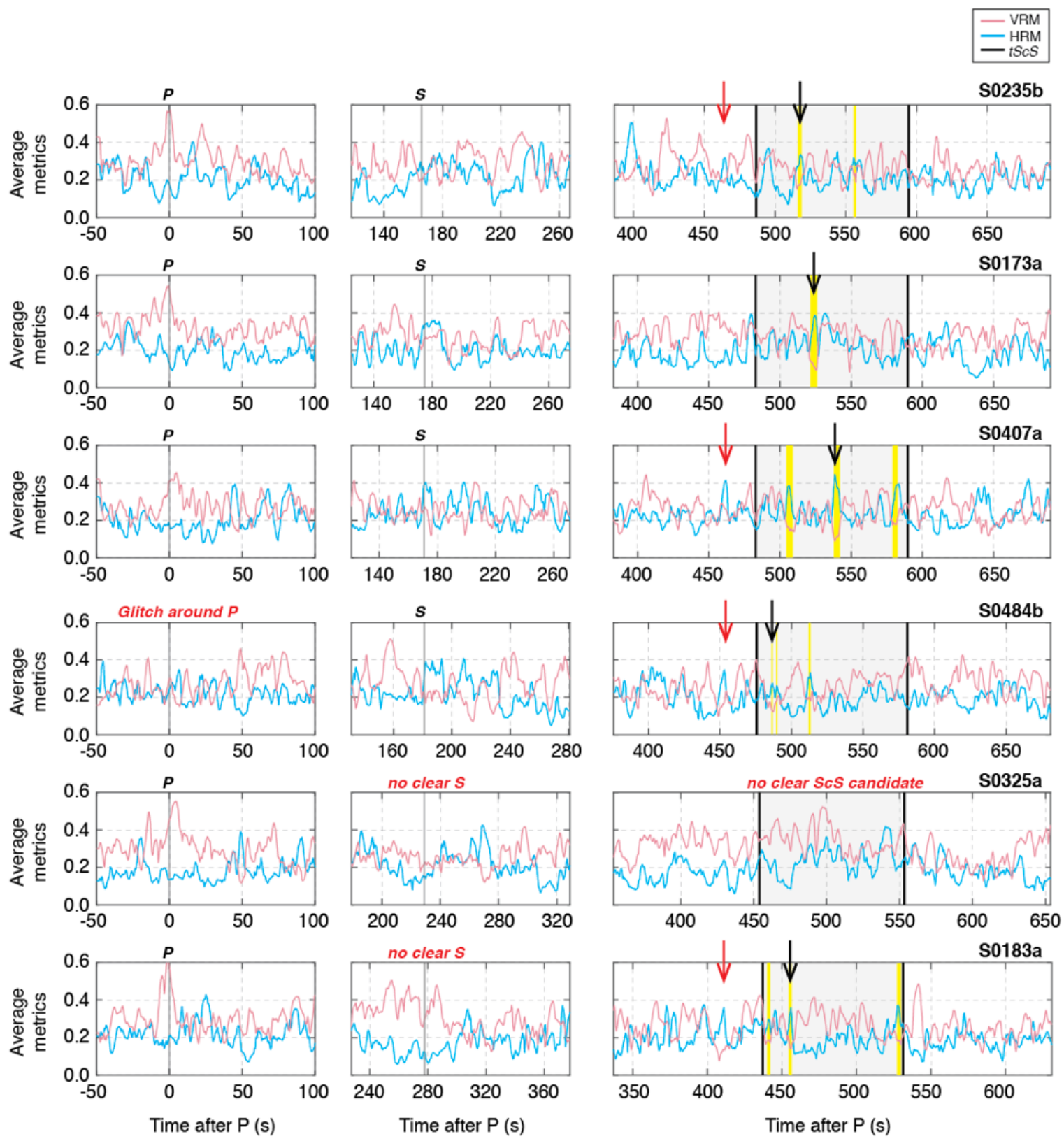

Fig. S2-4. Frequency dependent polarization analysis with six LF marsquakes.

The HRM and VRM metrics have been simplified by dropping the bolded cosine terms in Table S1. Gray vertical lines indicate the MQS P and S picks while pale gray shaded areas mark the $S c S$ predicted time window for selected Martian interior models with different core radii. Yellow bars indicate time ranges in which HRM maxima and VRM minima deviate more than 1-sigma from the mean HRM and VRM values within the $S c S$ time-window. There is a secondary phase that consistently arrives before the candidate $S c S$ phases on S0235b, S0407a, S0484a, and S0183a (red arrows). The $7^{\text {th }}$ analyzed marsquake, S0409d fails to satisfy our picking criteria for the $S c S$ candidate phase and is not shown. 


\begin{tabular}{|l|l|l|c|c|}
\hline \multicolumn{1}{|c|}{ Event } & \multicolumn{1}{|c|}{$\begin{array}{c}\text { ScS candidates - P Time } \\
\text { (HRM/VRM) }\end{array}$} & $\begin{array}{c}\text { Secondary } \\
\text { phases - P Time } \\
\text { (HRM/VRM) }\end{array}$ & \multicolumn{1}{c|}{$\begin{array}{c}\text { UTC for ScS } \\
\text { candidates }\end{array}$} & $\begin{array}{c}\text { MQS } \\
\text { Distance }\end{array}$ \\
\hline S0235b & $\mathbf{5 1 7 . 5 s}(1.9) / 557(1.6)$ & $463.5 \mathrm{~s}(1.7)$ & $2019072612: 27: 55.5$ & $28^{\circ}$ \\
\hline S0173a & $\mathbf{5 2 5 s ( 4 )}$ & $\mathrm{n} / \mathrm{a}$ & $2019052302: 31: 44.0$ & $29^{\circ}$ \\
\hline $\mathrm{S} 0407 \mathrm{a}$ & $507 \mathrm{~s}(2.6) / \mathbf{5 3 8 . 5 s}(5.0) / 581.5 \mathrm{~s}(2.2)$ & $462 \mathrm{~s}(1.6)$ & $2020011910: 06: 44.2$ & $29^{\circ}$ \\
\hline $\mathrm{S} 0409 \mathrm{~d}^{*}$ & $\mathrm{n} / \mathrm{a}$ & $\mathrm{n} / \mathrm{a}$ & $\mathrm{n} / \mathrm{a}$ & $30^{\circ}$ \\
\hline $\mathrm{S} 0484 \mathrm{~b}$ & $\mathbf{4 8 6 . 5 s}(2.2) / 513 \mathrm{~s}(1.8)$ & $452.5 \mathrm{~s}(2.0)$ & $2020040709: 00: 30.5$ & $31^{\circ}$ \\
\hline $\mathrm{S} 0325 \mathrm{a}$ & $\mathrm{n} / \mathrm{a}$ & $\mathrm{n} / \mathrm{a}$ & $\mathrm{n} / \mathrm{a}$ & $38^{\circ}$ \\
\hline $\mathrm{S} 0183 \mathrm{a}$ & $441.5 \mathrm{~s}(1.6) / \mathbf{4 5 6 s}(2.6) / 528 \mathrm{~s}(2.1)$ & $408 \mathrm{~s}(3.2)$ & $2019060302: 35: 25.0$ & $46^{\circ}$ \\
\hline
\end{tabular}

Table S2-2. Estimated phase arrivals for each event from frequency-dependent polarization analysis.

$\mathrm{ScS}$ times shown in bold are the preferred picks, with brackets indicating uncertainties on these numbers. 


\section{Method C: S-waveform cross correlation}

In this approach, we identify potential $\mathrm{ScS}$ arrivals in the coda of the $\mathrm{S}$ phase by taking advantage of the similarity between $\mathrm{ScS}$ and $\mathrm{S}$ waveforms. For this purpose, we cross-correlate an $\mathrm{S}$ phase template with the $\mathrm{S}$ phase coda. Any signal with a similar waveform to the $\mathrm{S}$ phase should produce a higher correlation at a lag-time which corresponds to the time difference between both signals. While the exact duration of the $\mathrm{S}$ phase is unknown, determining it precisely is not very important, because the teleseismic source function is composed of the $\mathrm{S}$ phase and near source reverberations which all propagate down to the core-mantle boundary (CMB) together. Hence, we also employ templates with extended durations that go beyond the $\mathrm{S}$ phase to include source-side reverberations. The principle is also utilized in seismic interferometry and receiver functions both of which can be expressed in terms of crosscorrelations (e.g., (97)).

The specific kind of cross-correlation we employ is phase cross-correlation (PCC, (98)) since it is amplitude unbiased to permit weak-amplitude signal detection. PCC works like a classical cross-correlation, but is based on instantaneous phases of analytic signals to measure the phase coherence. Below, we apply our analysis to the two quality A events: S0173a and S0235b.

\section{Results for event S0173a:}

(a)

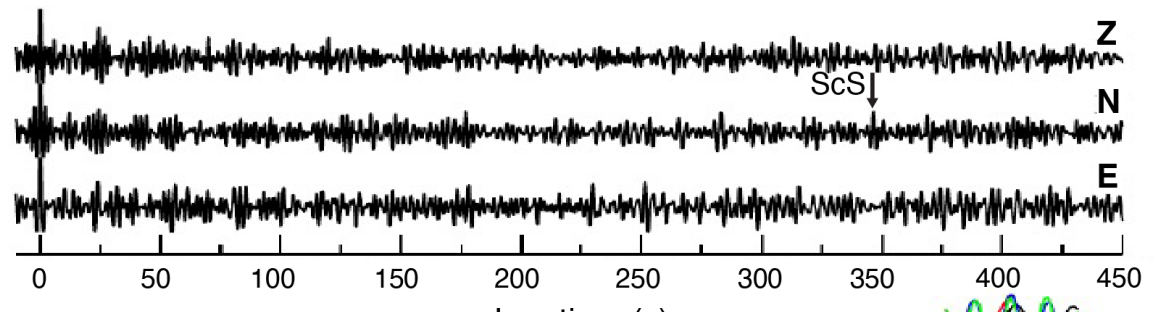

(b)

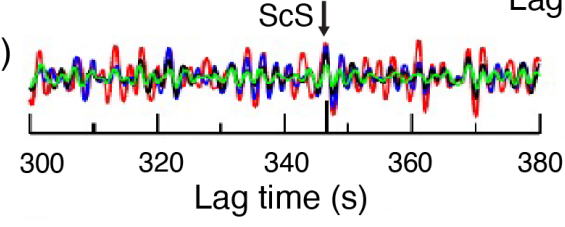

(c)
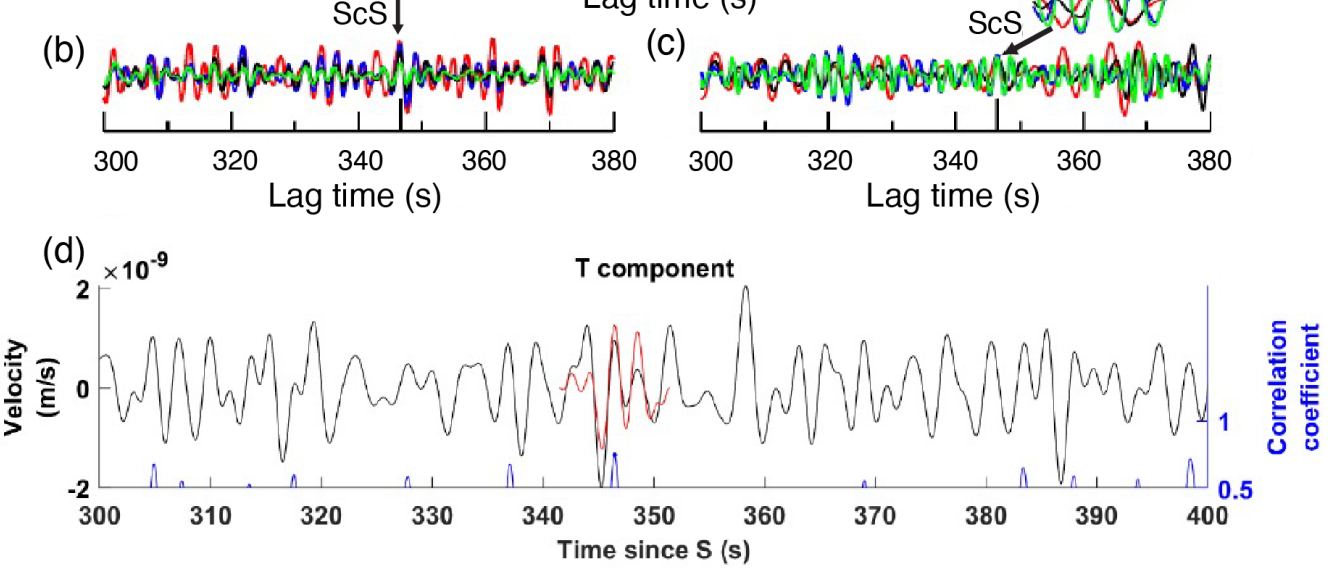

\section{Fig. S3-1. Correlation results for event S0173a.}

(a) Correlations of the $\mathrm{S}$ waveform and coda in a $[-5 \mathrm{~s}, 100 \mathrm{~s}]$ window with the same component record up to $450 \mathrm{~s}$ lag time. The ground velocity time series have been bandpassed between $0.15-$ $0.8 \mathrm{~Hz}$. The arrow marks a possible ScS arrival at $346.5 \mathrm{~s}$ lag time. (b) Same as (a) but showing a zoom of the N-component. Red, blue, black and green traces are for different window length of $25 \mathrm{~s}, 65 \mathrm{~s}, 105 \mathrm{~s}$, and $185 \mathrm{~s}$, respectively. The long vertical bar on the time axis marks the ScS 
arrival as in (a). The arrival at $346.5 \mathrm{~s}$ lag time appears as a stable feature. (c) Same as (b), but using 4 different 1-octave bandpass filters and a window length of $105 \mathrm{~s}$. Red, black, blue and green mark $0.15-0.3 \mathrm{~Hz}, 0.2-0.4 \mathrm{~Hz}, 0.3-0.6 \mathrm{~Hz}$ and $0.4-0.8 \mathrm{~Hz}$ frequency bands, respectively. The zoom shows the correlation waveforms from 342.5 to $350.5 \mathrm{~s}$ lag time. Frequency stability over a broad frequency band is apparent. (d) Black and red traces are the recording and $10 \mathrm{~s} \mathrm{~S}$ waveform, respectively. Correlation maxima are shown in blue. The $\mathrm{S}$ waveform has been shifted by $346.5 \mathrm{~s}$ as obtained through the correlation maximum (blue line). This correlation maximum happens at the same lag time as measured with PCC.

Figure S3-1 demonstrates correlation results for event S0173a. Based on polarization analysis (e.g., as described in Section 1), the backazimuth and distance of the marsquake (99) are about $91 \mathrm{deg}$ and $28.9 \mathrm{deg}$, respectively. Besides this polarization approach, we also apply another common polarization analysis (100) to the data and achieve similar results. Therefore, for the PCC analysis, the data have not been rotated into radial (R) and transverse (T), since the East (E) and North (N) components can be considered as R and T. We also compare our azimuth estimates to the wind azimuth, because winds can also cause ground deformation $(31,93)$. The wind azimuth around the $\mathrm{ScS}$ arrival time is bounded between 225 and 255 deg from the north, not close to our estimates. Thus, the signal we observe is not due to the wind.

The correlations from Figure $S 3-1 a$ have been obtained employing a frequency band pass from 0.15 to $0.8 \mathrm{~Hz}$ and computing the PCC for each component with a $105 \mathrm{~s}$ template. The template starts $5 \mathrm{~s}$ before the direct $\mathrm{S}$ arrival and has been taken from each individual component (ZNE). The arrow on the $\mathrm{N}$ component marks a possible $\mathrm{ScS}$ arrival by its correlation maximum at $346.5 \mathrm{~s}$. A zoom to this arrival is shown in Fig. S3-1b. The different colors mark correlations for different template length $(25 \mathrm{~s}, 65 \mathrm{~s}, 105 \mathrm{~s}, 185 \mathrm{~s})$. It can be seen that the arrival at about $346.5 \mathrm{~s}$ is quite stable with respect to template changes. Figure $S 3-1 c$ illustrates correlograms for templates of $65 \mathrm{~s}$ and 4 different one-octave bandpass filters $(0.15-0.3 \mathrm{~Hz}, 0.2-0.4 \mathrm{~Hz}, 0.3-0.6$ $\mathrm{Hz}, 0.4-0.8 \mathrm{~Hz}$ ). It can be seen that the signal at about $346.5 \mathrm{~s}$ lag time remains stable from about 0.15 to $0.8 \mathrm{~Hz}$. In fact, within the shown 300 to $380 \mathrm{~s}$ lag time window, it is the feature which shows coherent correlation maxima for the four frequency bands. Note that its lag time corresponds to the arrival identified by the polarization approach described in Section 1 .

In addition to PCC, we also adopt the classical cross-correlation. We apply a bandpass filter $(0.1-0.6 \mathrm{~Hz})$ to the transverse-component data. We then extract a 10-s SH waveform from the filtered data as the template and calculate the correlation coefficient between the template and the coda waves. The classical cross correlation provides a peak correlation coefficient at $346.5 \mathrm{~s}$, identical to the PCC result. We overlap the scaled SH waveform template on the coda wave corresponding to the peak and demonstrate that the coda waveform is in a similar shape as the template (Fig. S3-1d).

\section{Results for event S0235b:}

The second event, S0235b, occurred with a backazimuth and distance of $74 \mathrm{deg}$ and 27.8 deg (99). The polarization and correlation analyses are similar to the previous marsquake. For this event we find a signal which seems to be consistent with a possible ScS arrival (Fig. S3-2). The highest correlation is measured at about $341.1 \mathrm{~s}$ and has been marked by an arrow in Fig. S3$2 a$. Further, there exist no coexisting coherent signals on $\mathrm{Z}$ or $\mathrm{R}$ which strengthens our argument that this signal can be due to an $\mathrm{SH}$ wave. Also, this signal is stable with respect to variations of the template length (Fig. S3-2b) and appears coherently over the 0.05-0.6 Hz frequency band 
(Fig. S3-2c). In fact, it is the only signal with coherent correlation maxima within the shown lag time window and three frequency bands. The classical correlation approach $(0.3-0.7 \mathrm{~Hz}, \mathrm{Fig}$. S3$2 d$ ) provides a similar result, with a peak at $343.9 \mathrm{~s}$.

\section{(a)}
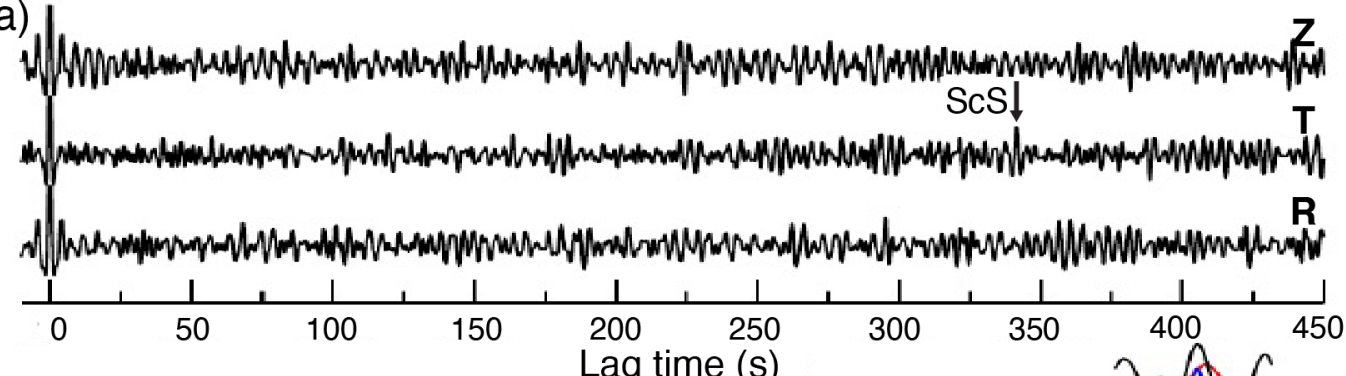

(b)

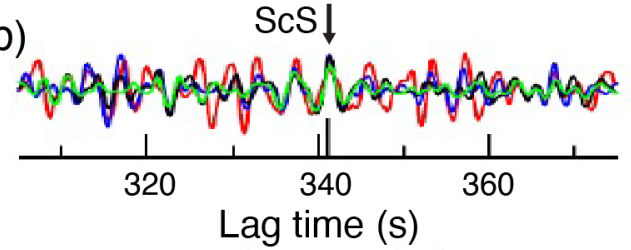

(c)

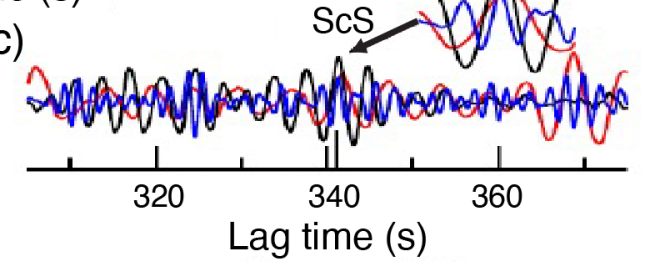

(d)

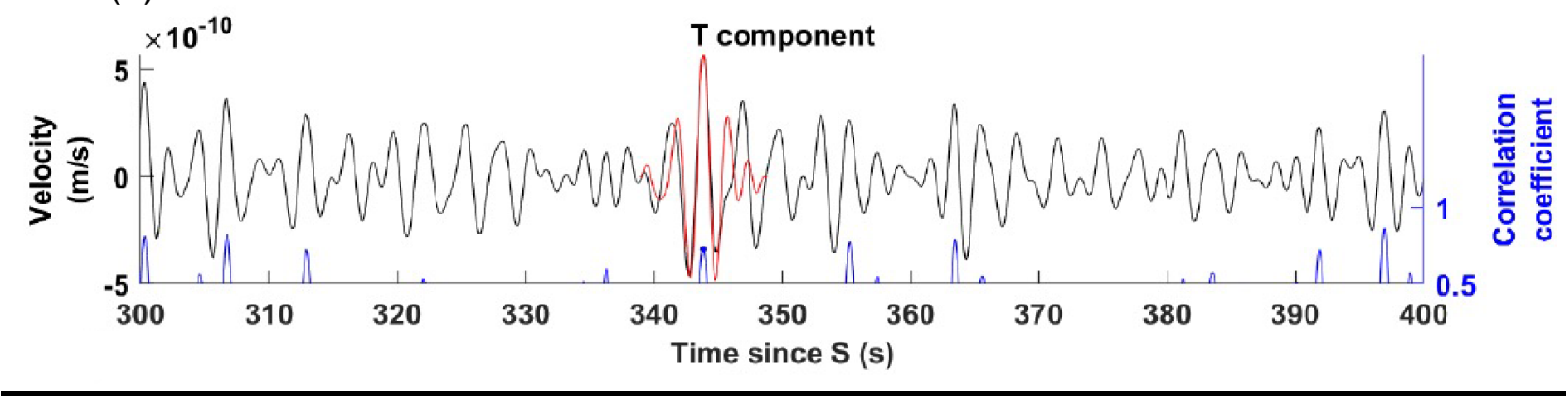

Fig. S3-2. Correlation results for event S0235b.

(a) Correlations of the $\mathrm{S}$ waveform and coda in a $[-5 \mathrm{~s}, 100 \mathrm{~s}]$ window with the same component record up to $450 \mathrm{~s}$ lag time. The ground velocity time series have been bandpassed between 0.15 $0.6 \mathrm{~Hz}$. The arrow marks a possible ScS arrival at $341.1 \mathrm{~s}$ lag time. (b) Same as (a) but showing a zoom of the T-component. Red, blue, black and green traces are for different window lengths of $25 \mathrm{~s}, 65 \mathrm{~s}, 105 \mathrm{~s}$, and $185 \mathrm{~s}$, respectively. The long vertical bar on the time axis marks the ScS arrival as in (a). The arrival at $341.1 \mathrm{~s}$ lag time appears as a stable feature. (c) Same as (b) using 3 different 1-octave bandpass filters and a window length of $105 \mathrm{~s}$. Red, black and blue mark $0.1-0.2 \mathrm{~Hz}, 0.2-0.4 \mathrm{~Hz}$, and $0.4-0.8 \mathrm{~Hz}$ frequency bands, respectively. The zoom shows the correlation waveforms from 337.1 to $345.1 \mathrm{~s} \mathrm{lag} \mathrm{time.} \mathrm{Frequency} \mathrm{stability} \mathrm{over} \mathrm{a} \mathrm{broad}$ frequency band is apparent. (d) Black and red traces are the recording and $10 \mathrm{~s} \mathrm{S-waveform,}$ respectively. Correlation maxima are shown in blue. The $\mathrm{S}$ waveform has been shifted by $343.9 \mathrm{~s}$ as obtained through the correlation maximum (blue line). 


\section{Method D: Event backazimuth estimation and detection of ScSH from correlation}

This section details the use of waveform cross-correlation to identify horizontally polarized seismic waves. It was developed independently to Method C, described in Section 4, and whilst the overriding principle is the same, the events analyzed, the precise processing choices, and the use of multiple estimates of coherence between different phases make this method distinct from Method C. The difference between the results ascertained for Methods C and D can be seen in the figures provided in Section 6, which reviews the picks made using different methods.

\section{Signal Processing Steps, $P$ and $S$ body wave picks:}

The $\mathrm{P}$ and $\mathrm{S}$ body wave picks are performed using the 3 SEIS-VBB components. Seismograms first have "tick noise" (regular signal repeating every second) and glitch waveforms are removed on the raw VBB data by using respectively the method described in (101) and the "UCLA" method described by (39). Then, instrument response is removed to obtain ground velocity and rotated to the vertical, North and East coordinate frame before P and $\mathrm{S}$ body wave picks are performed. Two main frequency bands are used: $0.4-1 \mathrm{~Hz}$ for $\mathrm{P}$ wave analysis, and $0.3-1 \mathrm{~Hz}$ for $\mathrm{S}$ wave analysis. The polarization of the ground velocity is computed in overlapping windows of $4 \mathrm{~s}$ length by both coherence and covariance methods. The instantaneous phase coherence (102) between the 3 components and the envelope of the filtered signals are also computed.

As seismic body waves are linearly polarized in an isotropic medium, we are search for signals with the following characteristics: high rectilinearity (from the polarization analysis), high amplitude (from the envelope of the signals), high instantaneous phase coherence between channels (vertical and horizontal for $\mathrm{P}$ and $\mathrm{S}$ waves). The $\mathrm{P}$ and $\mathrm{S}$ picks correspond to signals with an incidence angle roughly consistent with predicted $\mathrm{P}$ and $\mathrm{S}$ incidence. An example of the implementation of these criteria is provided in Fig. S4-1 for event S0235b.

\section{Selection of events:}

As $\mathrm{P}$ and $\mathrm{S}$ waves should arrive along the same azimuth, we compute the arrival azimuth of around $\mathrm{P}$ and $\mathrm{S}$ waves and use only events presenting arrival azimuth consistent between $\mathrm{P}$ and $\mathrm{S}$ wave trains.

The azimuth is estimated from $\mathrm{P}$ waves by assuming that the first 3 seconds of $\mathrm{P}$ wave is dominated by the $\mathrm{P}$ wave, whereas the first ten seconds of the $\mathrm{P}$ wave train (i.e., the ten seconds starting with the $\mathrm{P}$ wave arrival) are dominated by $\mathrm{P}$ and $\mathrm{SV}$ phases converted from $\mathrm{P}$ by crustal discontinuities. Therefore, we search for the horizontal direction that presents both the highest energy in the first ten seconds of $\mathrm{P}$ wave train, and a negative correlation with vertical component in the first 3 seconds.

The azimuth of $\mathrm{S}$ wave is estimated by assuming that the $\mathrm{S}$ wave train contains only $\mathrm{SV}$ and $\mathrm{SH}$ waves that are arriving at different times due to anisotropy along the ray path. Therefore, we search the horizontal direction that presents the highest positive correlation with the vertical component in the first few seconds of the $\mathrm{S}$ wave train.

When these two estimates are roughly consistent, the event is selected because our estimates $\mathrm{P}$ and $\mathrm{S}$ arrival times, as well as event backazimuth, are consistent. This consistency is reached for only 7 broadband and low frequency events. Event S0325a is not included in that list due to the lack of clear ScS arrival, leading to six useful events (table S4-1). Figure S4-2 presents the variations of the backazimuth markers as a function of the azimuth of the horizontal component selected for the computation for event S0235b. 


\section{ScS arrivals:}

The transverse component (perpendicular to the quake backazimuth previously estimated) is computed in the $0.3-1 \mathrm{~Hz}$ frequency range. By assuming that the waveform of the direct SH wave train, including depth phases, is similar to the $\mathrm{ScS}$ wave train, we search for peaks of correlation coefficients between the $\mathrm{SH}$ wave train and the rest of the transverse component records. These correlation peaks are indicated in Fig. S4-1(f) by arrows. These peaks provide some clues of where the ScS could be, however, because it does not contain any information on the amplitude, an $\mathrm{ScS}$ arrival is validated only if the transverse record contains a significant energy above the noise. An example is provided in Fig. S4-3 for the S0235b event.

(a)

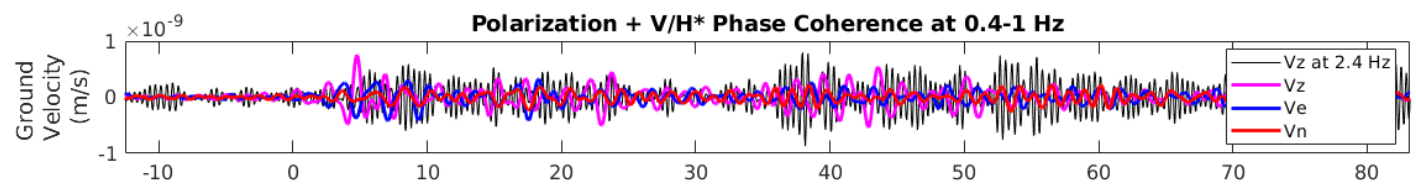

(b)

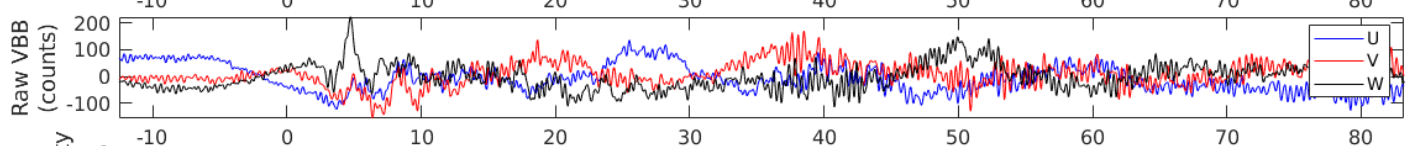

(c)
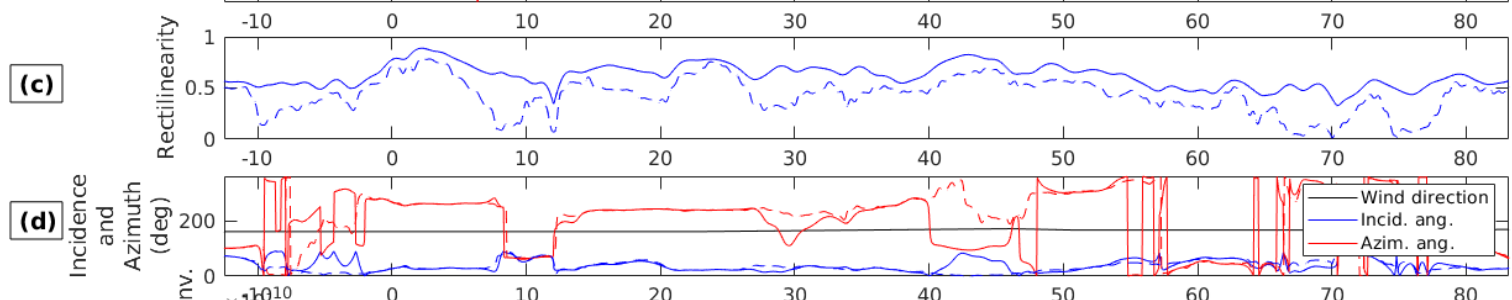

(e)

(f)

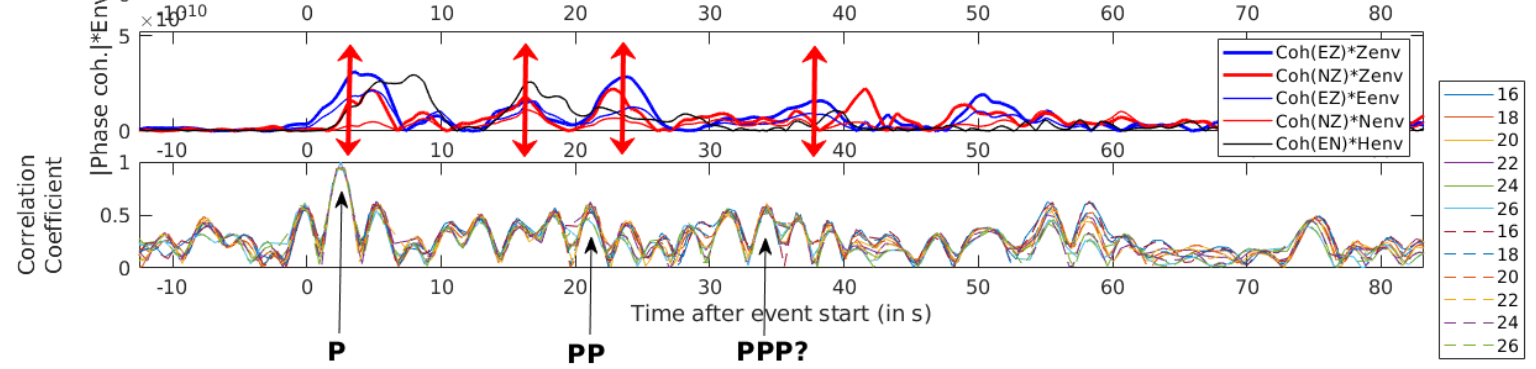

Fig. S4-1. Example of phase identification for event S0235b.

(a) Ground velocity of the vertical component in a narrow band around $2.4 \mathrm{~Hz}$ (black line) and of the three components in the 0.4-1 Hz frequency range (colors). (b) Raw VBB U, V and W components (in counts) low pass filtered below $3 \mathrm{~Hz}$. Rectilinearity is shown in panel (c), and incidence angle (blue) and azimuth (red) in panel (d) for polarization analysis with coherence (plain lines) and covariance (dashed lines) methods. Wind azimuth is indicated by a black line in panel (d), where blue circles indicate time periods for which polarization direction is along the wind direction. Panel (e) presents the products of instantaneous phase coherence between the vertical and the East and North in blue and red respectively, with the envelope of the vertical component (thick lines), or the corresponding horizontal component (thin lines). Coherence between East and North is shown in black. Panel (f) presents the maximum of correlation coefficient between $\mathrm{P}$ waveform and vertical component. arrow indicates the potential body wave arrivals, blue circles time periods for which the polarization direction is along wind direction. Black arrows indicate correlation maxima corresponding to multiple potential arrivals. 
(a)

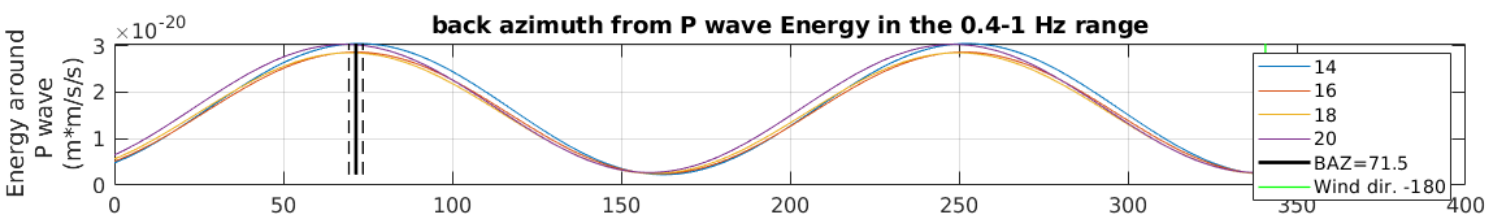

(b)
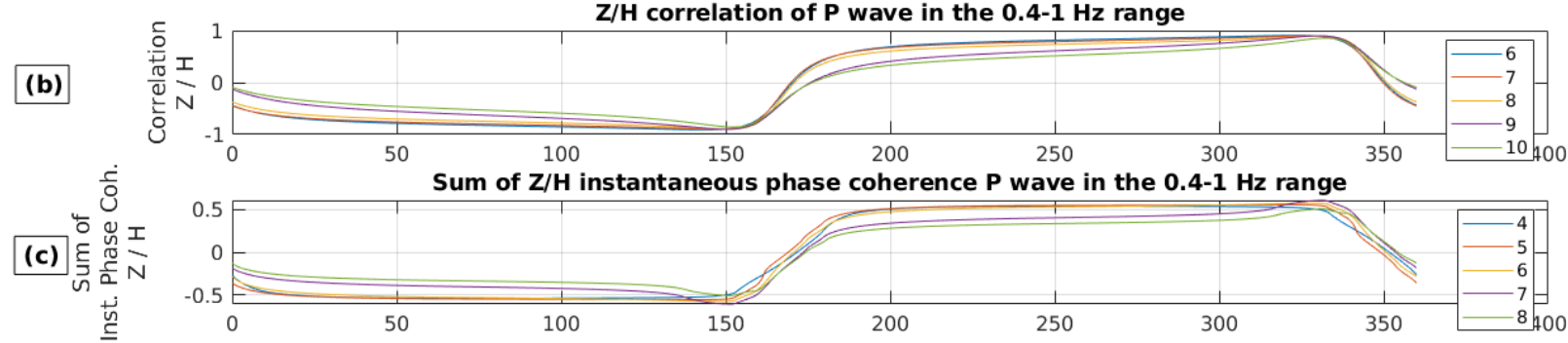

(d)
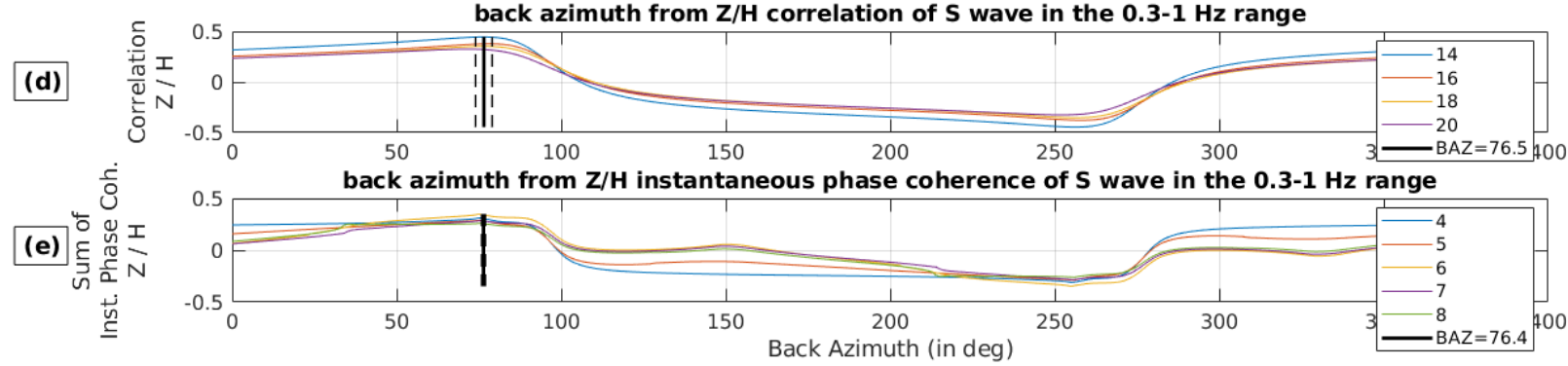

Fig. S4-2. Example of backazimuth identification for event S0235b.

From top to bottom: (a) energy along the horizontal component around the $\mathrm{P}$ wave arrival, (b) correlation coefficient between vertical and horizontal components around the $\mathrm{P}$ wave, (c) average of instantaneous phase coherence between vertical and horizontal component around the $P$ wave, (d) correlation coefficient between vertical and horizontal components around the $S$ wave, (f) average of instantaneous phase coherence between vertical and horizontal component around the $\mathrm{S}$ wave. For each panel, all possible backazimuths are examined and various window length (in seconds) are tested (different colors). Best estimates of backazimuth are identified by vertical black lines. 
(a)

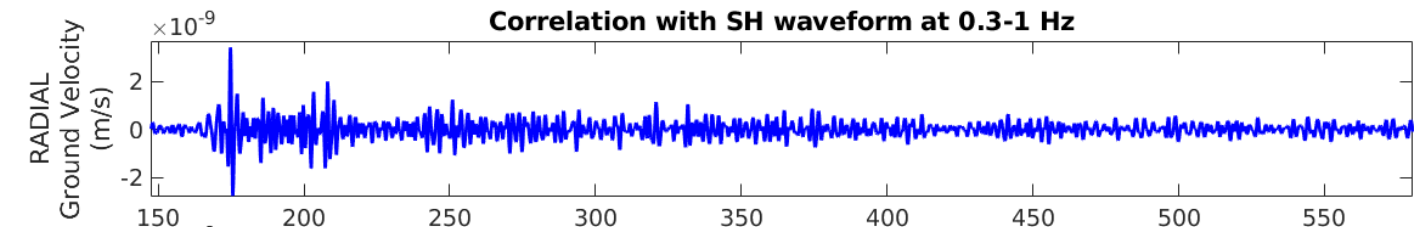

(b)

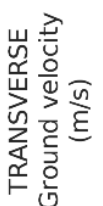

$\times 10^{-9}$

200

250

350

500

(c)

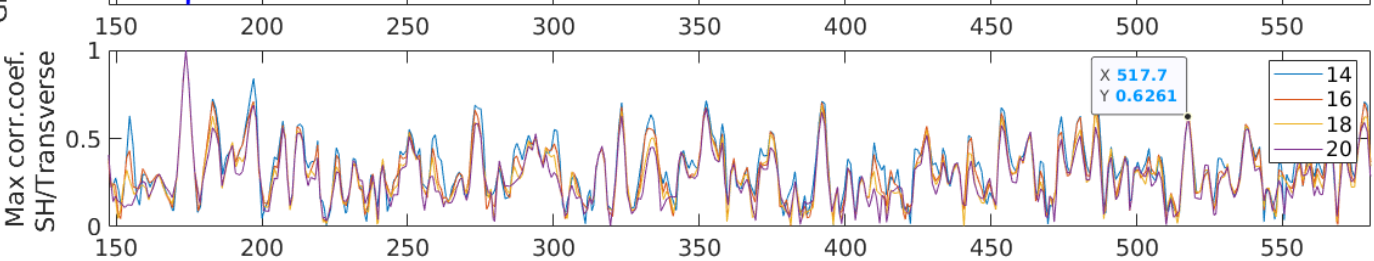

Time after event start (in s)

(d)

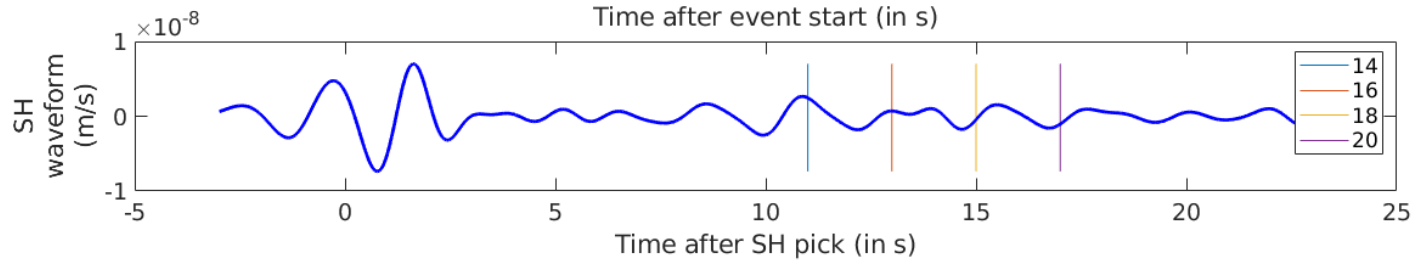

Fig. S4-3. Example of ScS identification for event S0235b.

From top to bottom, (a) horizontal ground velocity in the radial direction, (b) horizontal ground velocity in the transverse direction, (c) maximum correlation coefficients between $\mathrm{SH}$ waveform and horizontal transverse component for different sizes of SH window). (d) S wave arrival in the horizontal transverse direction (SH waveform), Vertical lines in (d) indicate the end of the SH wave window. A marker indicates at the time corresponding to a good correlation and the presence of energy along the transverse component (panel c), which we identify as ScS.

\begin{tabular}{|c|c|c|c|c|c|}
\hline Event & $P$ time [sec] & S time [sec] & Backazimuth [deg] & ScS-S time [sec] & Reference time (UTC) \\
\hline S0185a & 50.62 & 377.9 & 138.5 & 152.3 & $2019-06-05 T 02: 13: 25.115848 Z$ \\
\hline S0173a & 10 & 188.8 & 91.93 & 345.2 & $2019-05-23 T 02: 22: 48.546895 Z$ \\
\hline S0484b & 31.1 & 204.2 & 110.4 & 322.3 & $2020-04-07 T 08: 52: 05.561869 Z$ \\
\hline S0407a & 5 & 175.7 & 94.15 & 370 & $2020-01-19 T 09: 57: 43.268319 Z$ \\
\hline S0235b & 2.5 & 173.9 & 73.7 & 343.9 & $2019-07-26 T 12: 19: 16.991001 Z$ \\
\hline S0409d & 42.8 & 206 & 80 & 320.1 & $2020-01-21 T 11: 30: 42.679841 Z$ \\
\hline
\end{tabular}

\section{Table S4-1. ScS picks from joint SH-correlation and backazimuth analysis.}

Event label, $\mathrm{P}$ and $\mathrm{S}$ arrival times (in seconds relative to reference time), quake backazimuth (in degrees), ScS-S differential time (in seconds relative to reference time), and UTC reference time are provided. 


\section{Method E: Vespagram analysis of polarization filtered waveforms}

In contrast to the approaches described in previous sections, which are primarily applied to data from individual events, the approach employed in this section uses data from multiple events to create a vespagram which is used to infer the weighted average properties of the $\mathrm{ScS}$ signals. From there, appropriate travel times for each event's $\mathrm{ScS}$ arrival are calculated.

Here, we selected 8 quality A and B events (Table S5-1) from the Marsquake Service (MQS) catalog (99) to search for core-reflected phases (e.g., ScS). We first removed the instrument response to obtain seismograms in velocity and then rotated the 3 SEIS-VBB components from UVW to ZNE coordinates (28). The data were bandpass filtered between 0.2$0.8 \mathrm{~Hz}$ to remove the 1-Hz tick noise (103) as well as long-period noise. Seismic events on Mars are often characterized by strong scattering in the crust (31), thereby prohibiting the detection of core phases. To enhance the core phases, we applied a time-domain polarization filter (104) to enhance the horizontally polarized $\mathrm{ScS}$ phase and suppress the non-linearly or vertically polarized S-wave coda and noise. A similar polarization filter technique was employed to detect the core-reflected waves in the Apollo seismic data (24), where strong scattering obfuscated the core arrivals. We obtained the linearity and polarization directions of the particle motions by computing the largest eigenvalue and eigenvector of the covariance matrix of the threecomponent data. The linearity and polarization direction were subsequently combined as a pointby-point weight function which we applied to the seismograms.

We first applied the polarization filter in the ZNE coordinate to pick the P-arrivals on the vertical component (BHZ) based upon the reported travel-time picks from MQS (99). We derived the backazimuths of events by computing the ratio between BHN and BHE components of P-wave polarization direction. We rotated the horizontal components to radial (BHR) and transverse (BHT) components using the backazimuths of events (Table S5-1). We then applied the polarization filter again in the ZTR coordinate to pick the S-arrivals on the transverse component. We normalized and aligned the S-waves at their maximum envelope amplitudes and ordered them by S-P time which is equivalent to epicentral distance (Fig. S5-1a). We computed the ratio between the envelope amplitude of the S-wave signal and mean envelope amplitude of a noise time window (300-500 s before S-arrival) as the signal-to-noise ratio (SNR) of S-waves (Table S5-1). We selected the noise time window to avoid any contaminations by instrument glitches (39). We scaled the transverse data near each ScS time window by multiplying amplitude by the SNR to give larger weights to the Quality A events (S0173a and S0235b) in the stack (Fig. S5-1b). Note that after SNR rebalancing of amplitudes, S0173a shows the largest amplitude and can potentially dominate the stacking result.

We used a vespagram approach adapted for the source array configuration on Mars to stack the polarization filtered envelopes on the transverse component (105). The goal is to search for coherent energy with horizontal polarization near the predicted slowness and time window of the ScS phase. We removed the known instrument glitches by zeroing the amplitudes in the reported glitch time windows (39). We converted S-P time to epicentral distances based on a pre-landing model (TAYAK model (106)) and used the average distance of these events as the reference distance (34.4 degrees). We computed the predicted travel-time and slowness of $\mathrm{ScS}$ at the reference distance using the TAYAK model (where the core radius is $1793.5 \mathrm{~km}$ ) assuming a source depth of $30 \mathrm{~km}$. The transverse component vespagram reveals several coherent energy packets near the $\mathrm{ScS}$ arrival time prediction (Fig. S5-2). The highest amplitude feature of the plot reveals the dominance of the S0173 event in this time window (Fig. S5-1b). We picked the 
highest amplitude energy within a $\pm 15 \mathrm{~s}$ window of the ScS prediction (slowness $=-8.2 \pm 0.5$ $\mathrm{s} / \mathrm{deg}$, time $=285.6 \pm 5.0 \mathrm{~s}$ ) as our candidate $\mathrm{ScS}$ phase (Fig. S5-2). We then used the slowness and travel-time measurements at the reference distance to predict the travel-time of $\mathrm{ScS}$ across the entire distance range of these events (Fig. S5-1b). The vespagram predictions were then used to guide our picks on the individual $\mathrm{ScS}$ arrivals by selecting the largest amplitudes within $15 \mathrm{~s}$ near the predicted vespagram arrival time (Fig. S5-1b). The vespagram predicted travel-time is dependent on the choice of pre-landing models, but the differences caused by different models are much smaller than the 15-s time window used for the ScS arrival picks. Therefore, our ScS picks are independent from the model selections. We summarize the resulting $\mathrm{ScS}$ travel-time picks in Table S5-1.

\begin{tabular}{|l|l|l|l|l|}
\hline Event & S-P Time $(\mathrm{s})$ & ScS-P Time $(\mathrm{s})$ & Backazimuth (deg) & SNR \\
\hline S0185a & $360.4 \pm 2.5$ & $444.9 \pm 5.0$ & $161.0 \pm 4.1$ & 5.6 \\
\hline S0183a & $305.6 \pm 2.5$ & $459.9 \pm 5.0$ & $99.8 \pm 2.8$ & 3.0 \\
\hline S0325a & $230.2 \pm 2.5$ & $500.3 \pm 5.0$ & $107.7 \pm 7.6$ & 9.0 \\
\hline S0484b & $173.4 \pm 2.5$ & $524.9 \pm 5.0$ & $118.5 \pm 11.8$ & 7.9 \\
\hline S0173a & $172.7 \pm 2.5$ & $517.9 \pm 5.0$ & $87.3 \pm 1.3$ & 37.5 \\
\hline S0235b & $171.1 \pm 2.5$ & $517.2 \pm 5.0$ & $81.8 \pm 3.1$ & 90.1 \\
\hline S0407a & $170.9 \pm 2.5$ & $523.8 \pm 5.0$ & $118.9 \pm 29.5$ & 8.6 \\
\hline S0409d & $164.1 \pm 2.5$ & $514.2 \pm 5.0$ & $102.2 \pm 50.3$ & 6.7 \\
\hline
\end{tabular}

Table S5-1. Summary of ScS travel-time picks, event backazimuths and SNR from Vespagram analysis. 
(a)
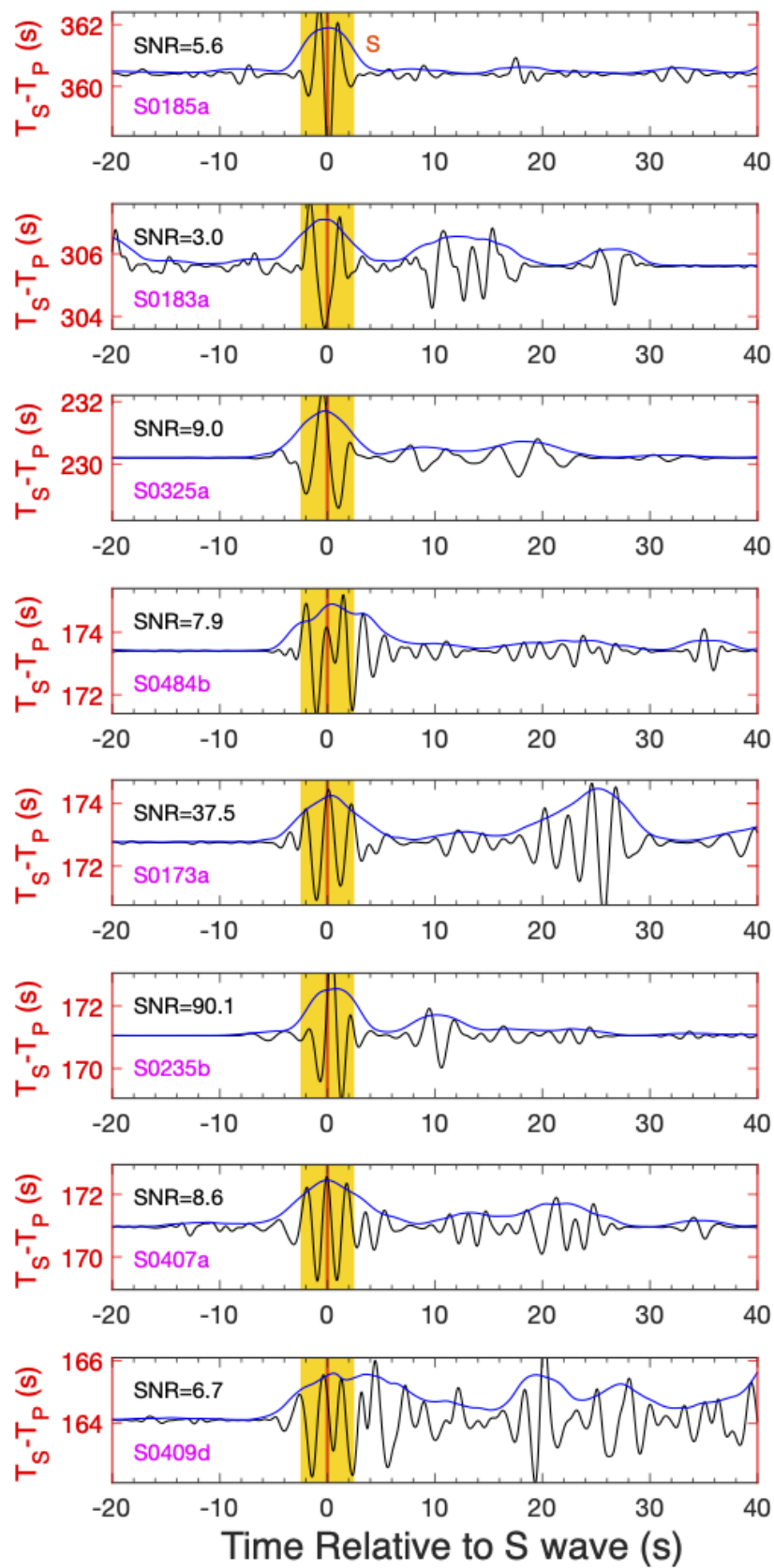

(b)

ScS Picks
Vespagram Predictions -
Model Predictions
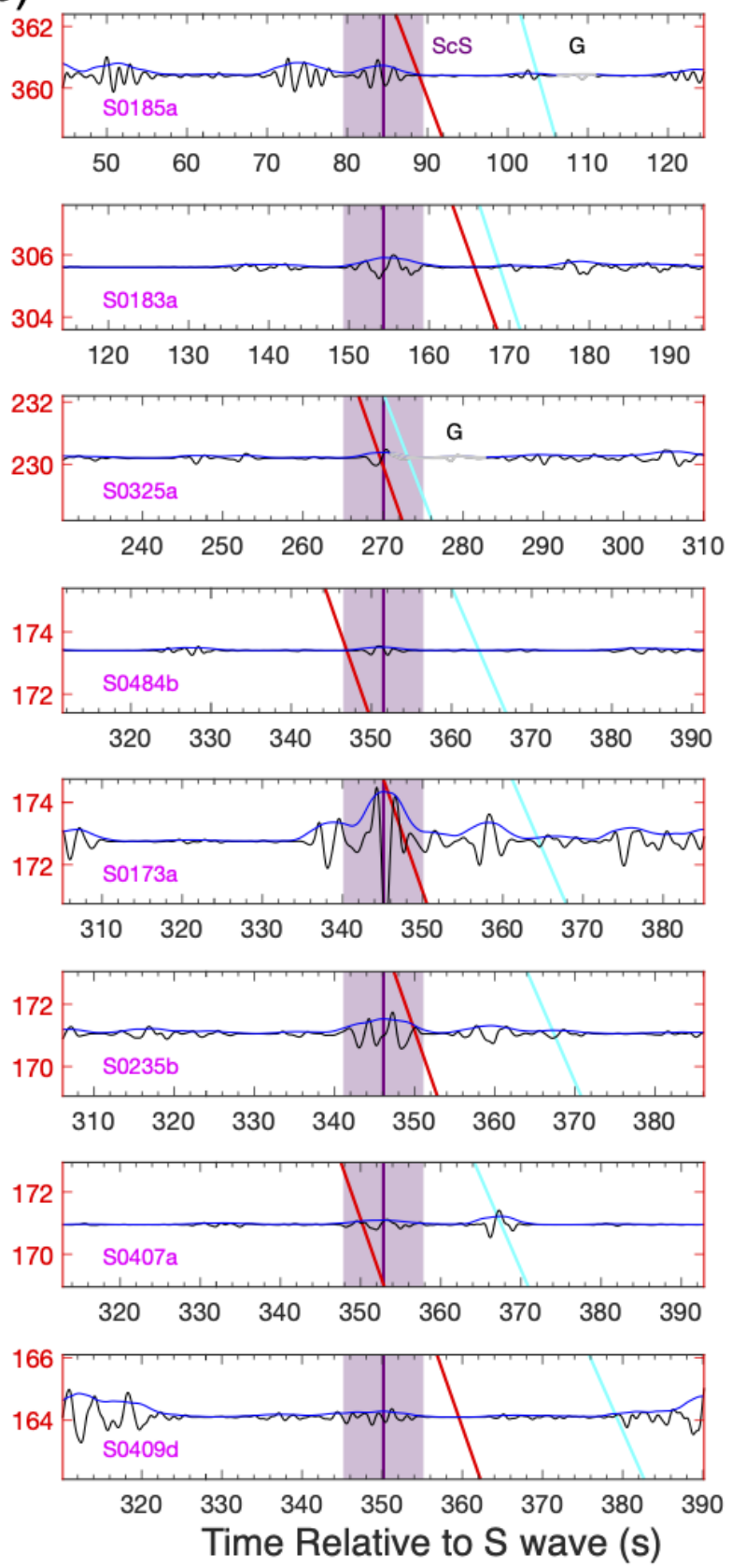

Fig. S5-1. Polarization filtered waveforms as input for the vespagram analysis. Polarization filtered waveforms and envelopes for (a) S and (b) ScS phases on the transverse component. The black and blue curves represent the polarization filtered waveforms and envelopes respectively. The red and cyan lines in (b) denote the predicted $\mathrm{ScS}$ travel-time curves based on this section's vespagram analysis and the TAYAK model respectively, and their slopes show the move-out of ScS phase. The orange lines in (a) and purple lines in (b) represent the travel-time picks of $\mathrm{S}$ and $\mathrm{ScS}$ phases respectively. The amplitudes in (b) are multiplied by SNR of $\mathrm{S}$ waves as described in this section. The uncertainties of $\mathrm{S}$ and $\mathrm{ScS}$ travel-time picks $(2.5 \mathrm{~s}$ 
for $\mathrm{S}, 5.0 \mathrm{~s}$ for $\mathrm{ScS}$ ) are shown as yellow and purple shaded regions. "G" indicates the presence of known instrument glitches (39), which are also highlighted as faded gray sections of the waveforms. Note that the ScS pick of S0325a event could be contaminated by a glitch.

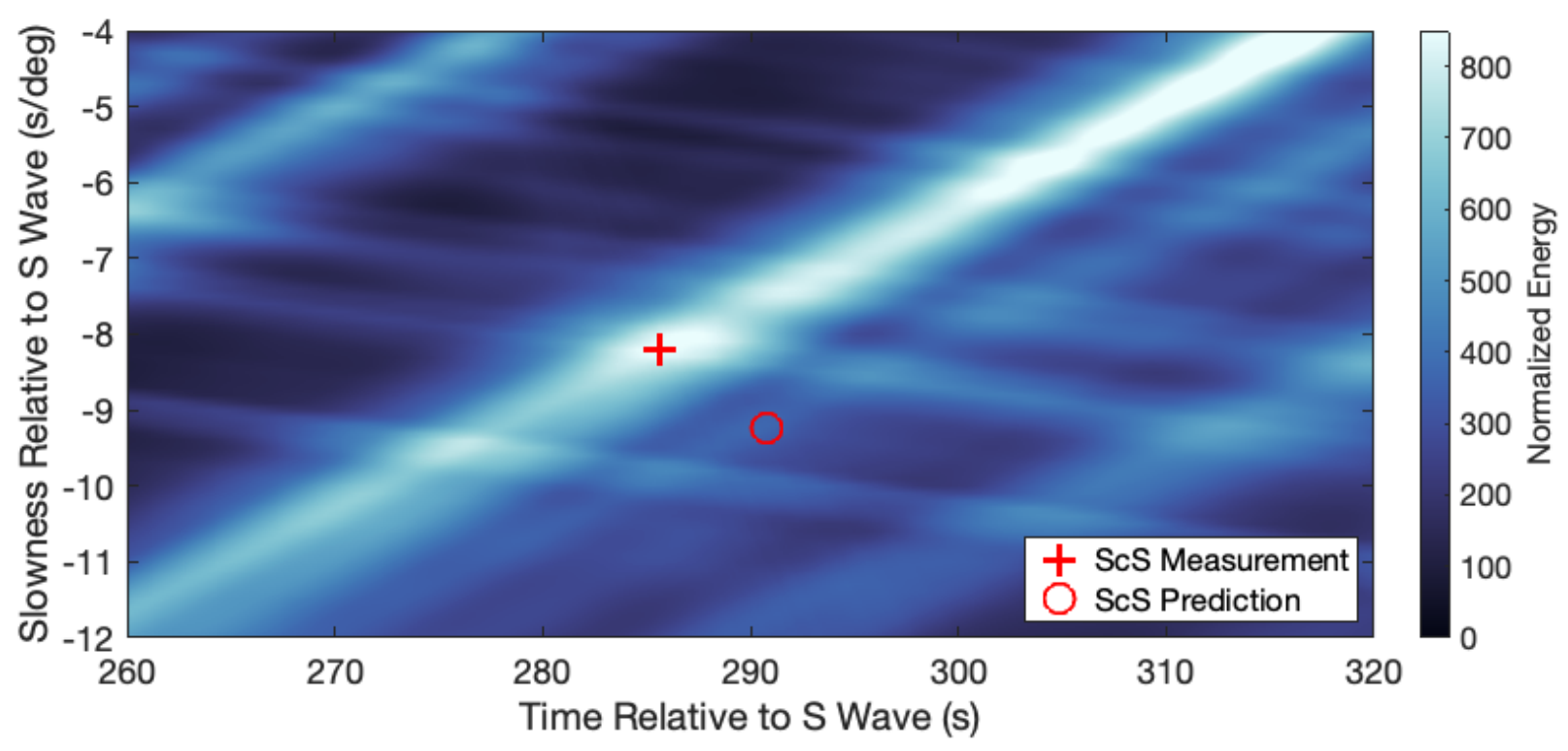

\section{Fig. S5-2. Vespagram analysis of the ScS phase.}

This vespagram is constructed from the envelopes of polarization filtered waveforms on the transverse component (BHT). The red circle denotes the predicted $\mathrm{ScS}$ slowness and travel-time at the reference distance ( 34.4 degrees) and $30 \mathrm{~km}$ source depth at using the TAYAK model. The red cross highlights the relative slowness $(-8.2 \pm 0.5 \mathrm{~s} / \mathrm{deg})$ and travel-time $(285.6 \pm 5.0 \mathrm{~s})$ measurements of $\mathrm{ScS}$ from the data. 


\section{Consolidation of phase picking}

This section presents a review of the ScS pick times described in the previous supplementary sections $1-5$. The review seeks to recognize energy arrivals in the waveform that correspond to the $\mathrm{ScS}$ phase, taking into account experience with Martian seismic phase arrivals, and rule out artefacts that could be mistaken for phase arrivals.

To test the spectral character of the phases the spectrum of each arrival's time window has been computed using a multi-taper approach (107). The spectrum is fit using a model combining attenuation and a source spectrum:

$$
A(f)=A_{0} A_{\text {src }}(f) A_{\text {att }}(f)
$$

Figure S6-1B shows spectra of $3 \mathrm{ScS}$ phase picks, compared with the S-wave spectrum of the distant-most event observed so far (S0167b, which is outside the distance where $\mathrm{ScS}$ can be wellobserved). Figure S6-1A shows $\mathrm{A}_{0}$, the amplitude extrapolated to static displacement. Generally, $\mathrm{ScS}$ are 10-20 dB in power below S, i.e., a factor of less than 10. Figure S6-2 compares this to ray-theoretical amplitudes, relative to the direct S-wave. These are computed using geometrical spreading, but without taking attenuation, source radiation pattern, scattering or reflections at other interfaces than the CMB into account and are therefore an upper limit of the relative $\mathrm{ScS}$ amplitude. Our detection of ScS energy implies the presence of a liquid core because the low relative amplitude of reflections predicted for a solid core would render $\mathrm{ScS}$ undetectable.

To recognize significant arrivals and understand differences in picks from the various methods, each waveform is plotted as the envelope of a filter bank of the relevant component: transverse if a backazimuth was determined, or sum of horizontal envelopes otherwise. The filter banks (Figs. S6-3 to S6-21) consist of half-octave wide acausal Butterworth bandpass filters of order 4 with center frequency in steps of a quarter octave. Using a multi-taper approach, the spectrum of a 20 second time window around the selected pick is computed and compared with the expected, correcting for source and attenuation. The Brune source was estimated (following (108)) by

$$
A_{\mathrm{src}}(f)=\frac{1}{1+\left(f / f_{c}\right)^{2}}
$$

with the corner frequency $f_{c}=1 \mathrm{~Hz}$, consistent with (29), so that the spectral shape is mainly controlled by attenuation, described by an attenuation term

$$
A_{\text {att }}(f)=\exp \left(-\pi f T / Q_{\text {eff }}\right)
$$

where $T$ is the estimated travel time of the S-wave and $Q_{\text {eff }}$ is an effective quality factor used to explain the spectral shape. For all events, we determine attenuation values compatible with previous values (29). In each case the spectral fits are shown alongside the filter bank for the arrival (Figs S6-3 to S6-21).

To exclude noise artefacts, we only accept picks with spectral decay matching that of the Swave of the distant-most marsquake in our dataset (see Fig. S6-1).

The two most important noise artefacts are:

1. Wind gusts. These excite a long-period signal due to induced tilt with a slope of $1 / \omega^{2}$, as well as high-frequency noise due to turbulence at the lander and the seismometer. The wind present during the arrivals documented in this paper is examined in section 8 of this supplement. 
2. Glitches, which are temperature-stress induced motions of SEIS or the tether, which have a long-period component only.

Actual phase arrivals are typically limited to an intermediate frequency range of $0.125-2$ $\mathrm{Hz}$ for the largest ones (the P and S-wave of S0235b), or much less, e. g. 0.4-0.8 Hz for secondary body waves or the P-waves of some small events (S0409d and S0407a). We therefore exclude all phase candidates that are accompanied by either an energy burst above $1.5 \mathrm{~Hz}$ or a pulse at the longest periods below $0.25 \mathrm{~Hz}$. This criterion must be handled with care however; some clear phases (the P-wave of S0173a most prominently) are immediately followed by a glitch, which we interpret as triggered relaxation of stress inside the instrument. Glitches are typically zero-phased signals, so they are easy to recognize in the envelope filter bank. As the S0173a P-wave shows; energy at intermediate frequencies some seconds before a glitch can actually be independent of it.

Up to seven different picking methods are deployed to identify seismic arrivals, including $\mathrm{ScS}$. The methodological details of are given in the preceding sections of this supplement. For each phase the picks are collectively reviewed and one pick with uncertainty is chosen for each event; these are the picks shown in table 1 of the main text.

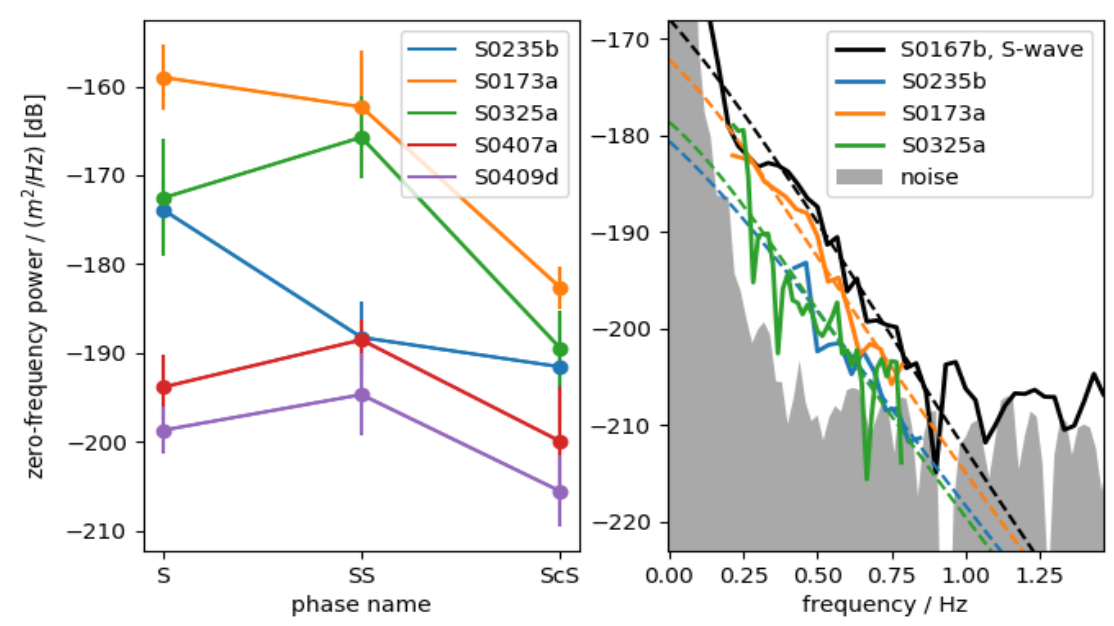

Fig. S6-1. Amplitudes and spectra of $3 \mathrm{ScS}$ picks (transverse component), compared to the teleseismic S-wave of a distant event (S0167b).

Left: The amplitudes show a typical S/ScS ratio of 10-20 dB in power for the high SNR events (S0173a, S0235b and S0325a), while the events S0407a and S0409d have such low SNR that the amplitude estimate is dominated by noise and thus unreliable.

Right: For the three larger events, ScS can be observed between 0.25 and $0.75 \mathrm{~Hz}$, with a spectral slope that is consistent with S-wave picks for teleseismic events (S0167b, see (32)).

Since the S-wave travel time of S0167b is 900 seconds, vs. 800 for ScS of the 3 other events, all phases were affected by the same average attenuation. The figure shows the frequency window in which $\mathrm{ScS}$ is expected to be observed $(0.3-0.8 \mathrm{~Hz})$ above the noise. The noise curve is taken from a 10-minute time window before event S0325a and is representative for the noise during all 4 events. 

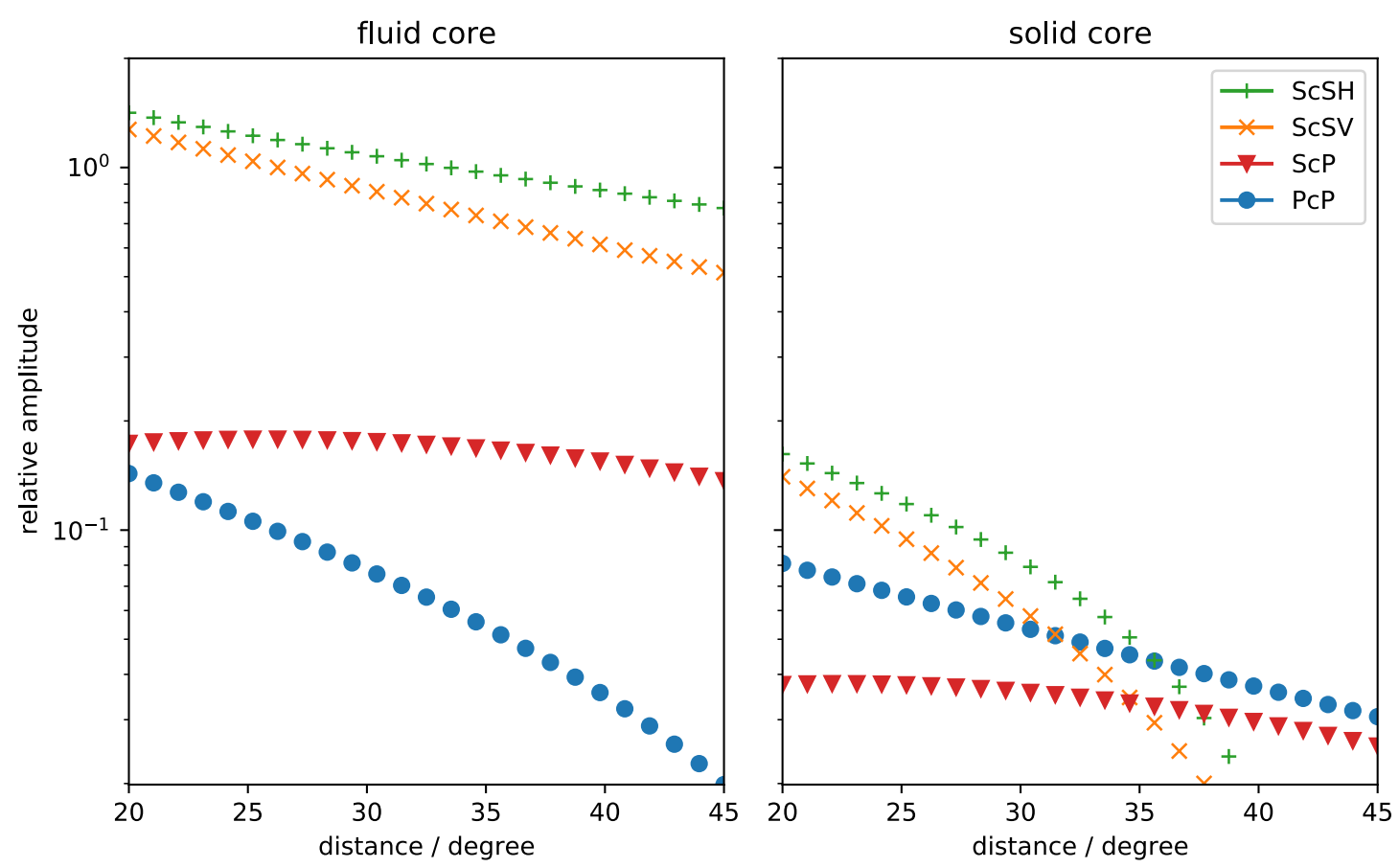

\section{Fig. S6-2. Ray-theoretical amplitudes of core-reflected phases for solid and fluid outer core}

The figure shows ray-theoretical amplitudes, based on geometrical spreading (109) and the reflection coefficient at the core-mantle boundary. The curves show that at the distance of our events, $\mathrm{ScSH}$ and $\mathrm{ScSV}$ have comparable amplitudes from ray theory alone. Note that $\mathrm{ScSV}$ can lose energy to $\mathrm{P}$-conversion at other interfaces than the $\mathrm{CMB}$. ScP is predicted to have an amplitude six times smaller than $\mathrm{ScSH}$, PcP less than a tenth of $\mathrm{ScSH}$.

For the solid core model, a shear modulus of $100 \mathrm{GPa}$ was assumed. All CMB-reflected phases would be reduced by a factor of at least ten in amplitude compared to the case of a fluid outer core at 30-degree distance. 

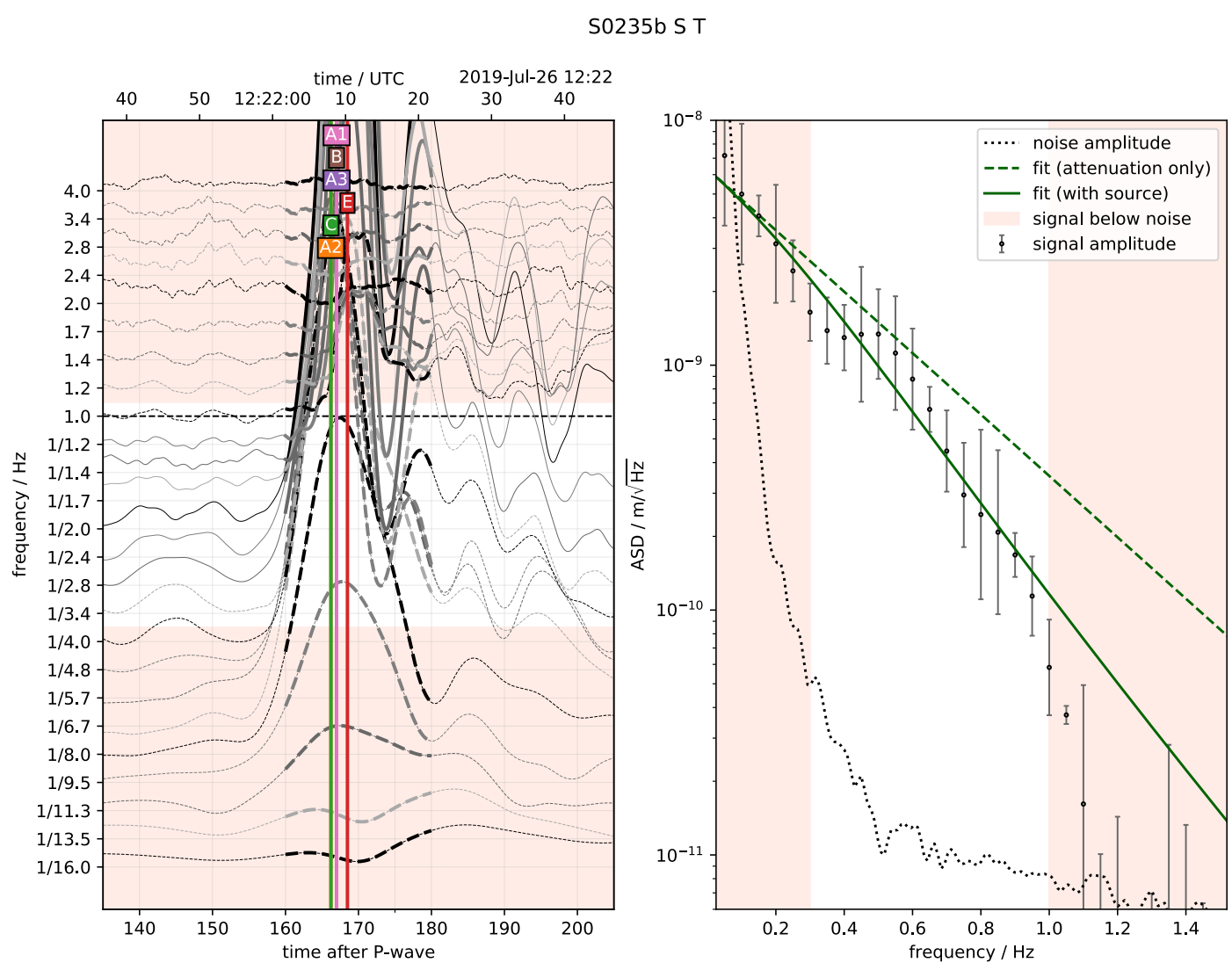

\section{Fig. S6-3. Filter bank for S0235b - S-phase}

Filter bank for the transverse component of the S-wave time window of event S0235b (left) and a spectral fit for the phase. Colored vertical lines mark the individual picks using the methods described in S1-S5. The red areas mark the frequency range, in which we estimate the signal to be not above the noise or polluted by other effects.

This pick is one of the clearest in the whole InSight dataset and there is consensus between the teams. Note that the 2 second offset in the vespagram based picks has to be taken into account when converting the other picks for this event into tScS-tP times.

Technical details: The left subplot shows envelopes of data filtered using half-octave wide acausal Butterworth bandpass filters of order 4 with center frequency in steps of a quarter octave. Each passband is normalized to the $95^{\text {th }}$ percentile individually. Solid bold lines mark the time and frequency windows used for the spectral fit in the right subplot. Dashed bold lines are outside the passband marked in the right subplot, thin lines are outside the time window used for spectral estimation. This time window has been manually chosen to avoid spurious signals.

The right subplot shows the result of a spectral estimate using the multi-taper method, as implemented by (107), with the one-sigma uncertainty of this estimate. The dashed line marks the spectrum of a noise window before the event. For selection of this noise window, see (99). The solid green line shows a spectral fit using a combined Brune source plus attenuation model (see text), the dashed green line shows the same fit when removing the source spectrum. 


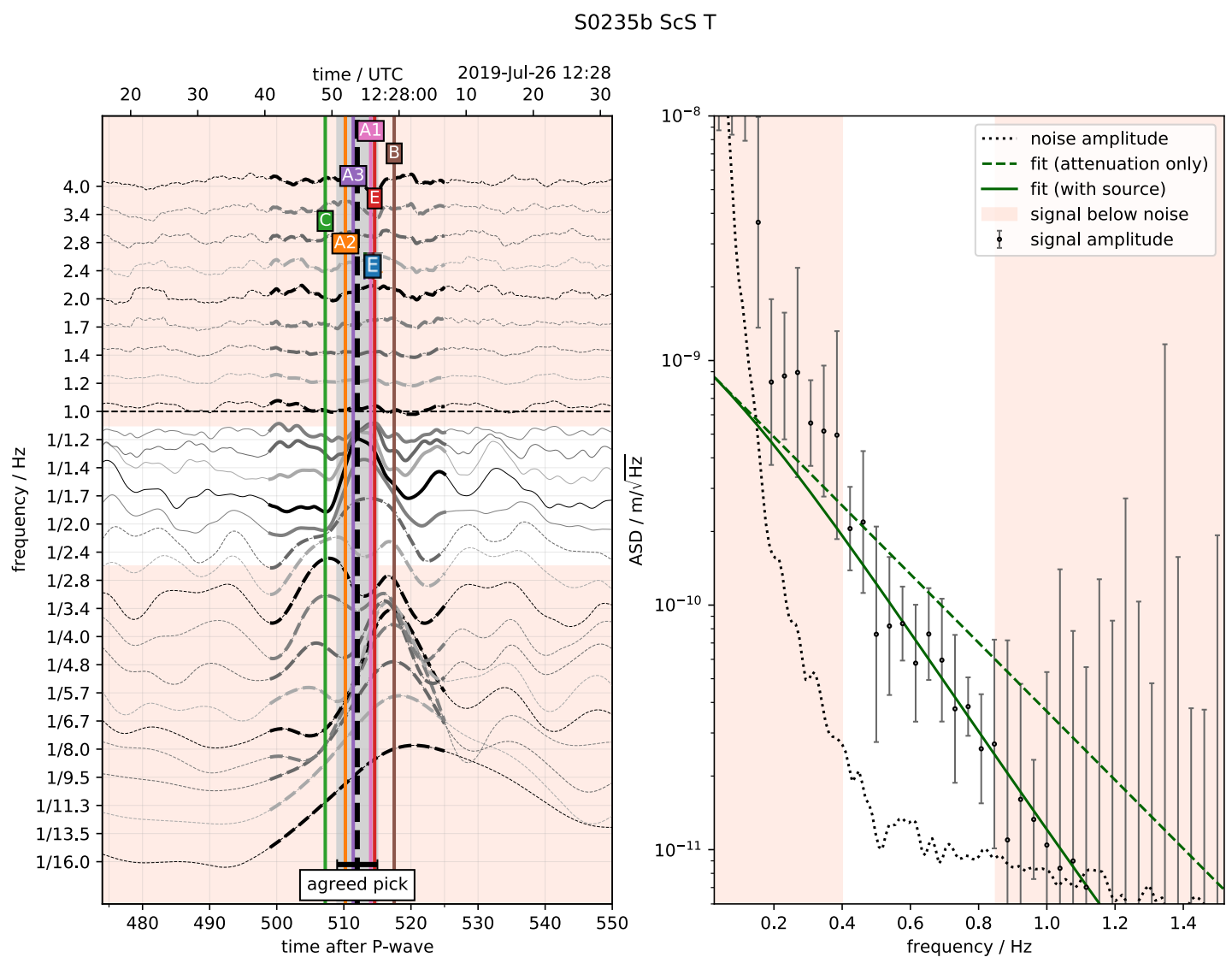

\section{Fig. S6-4. Filter bank for S0235b - ScS-phase}

The spread for this pick is 8 seconds between the teams. The polarization-based picks of ETH (A1, A3) and IPGP (A2, section S1) are based on the peak energy between 1.2 and 2 second period; the similarity/correlation-based method $\mathrm{C}$ picks the slightly earlier peak between 2 and 4.8 seconds. The vespagram-based method $\mathrm{E}$ is focusing on the later, long-period peak at 4 seconds and below. This latter peak has a frequency spectrum not compatible with the $\mathrm{S}$ wave spectrum and therefore more likely related to a wind burst. We pick at 511 seconds with an uncertainty of 3 seconds.

The black dashed line marks the consolidated pick; the error bar at the bottom shows its uncertainty. 

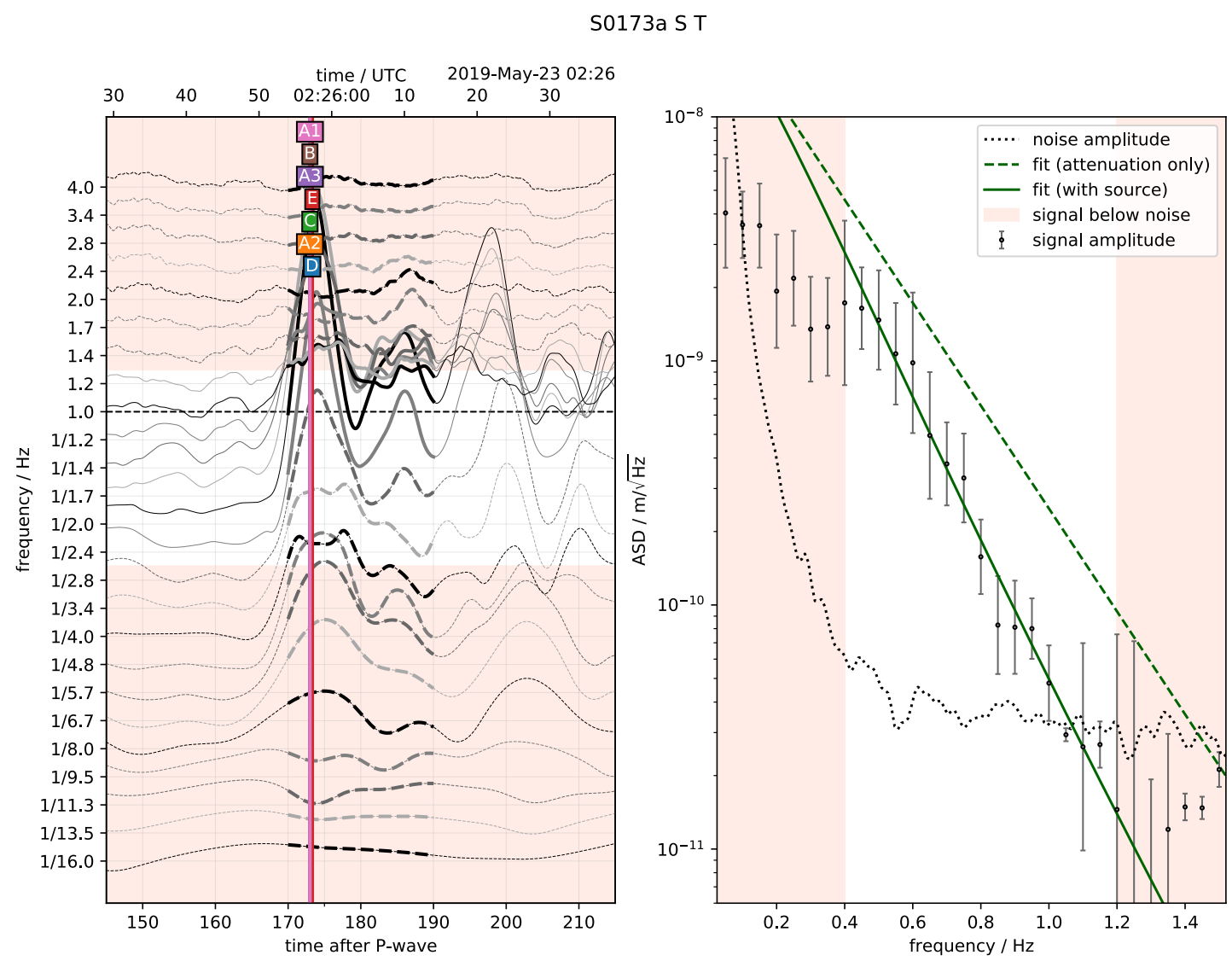

Fig. S6-5. Filter bank for S0173a - S-phase

This pick is one of the clearest in the whole InSight dataset and is the different methods produce consistent picks. 


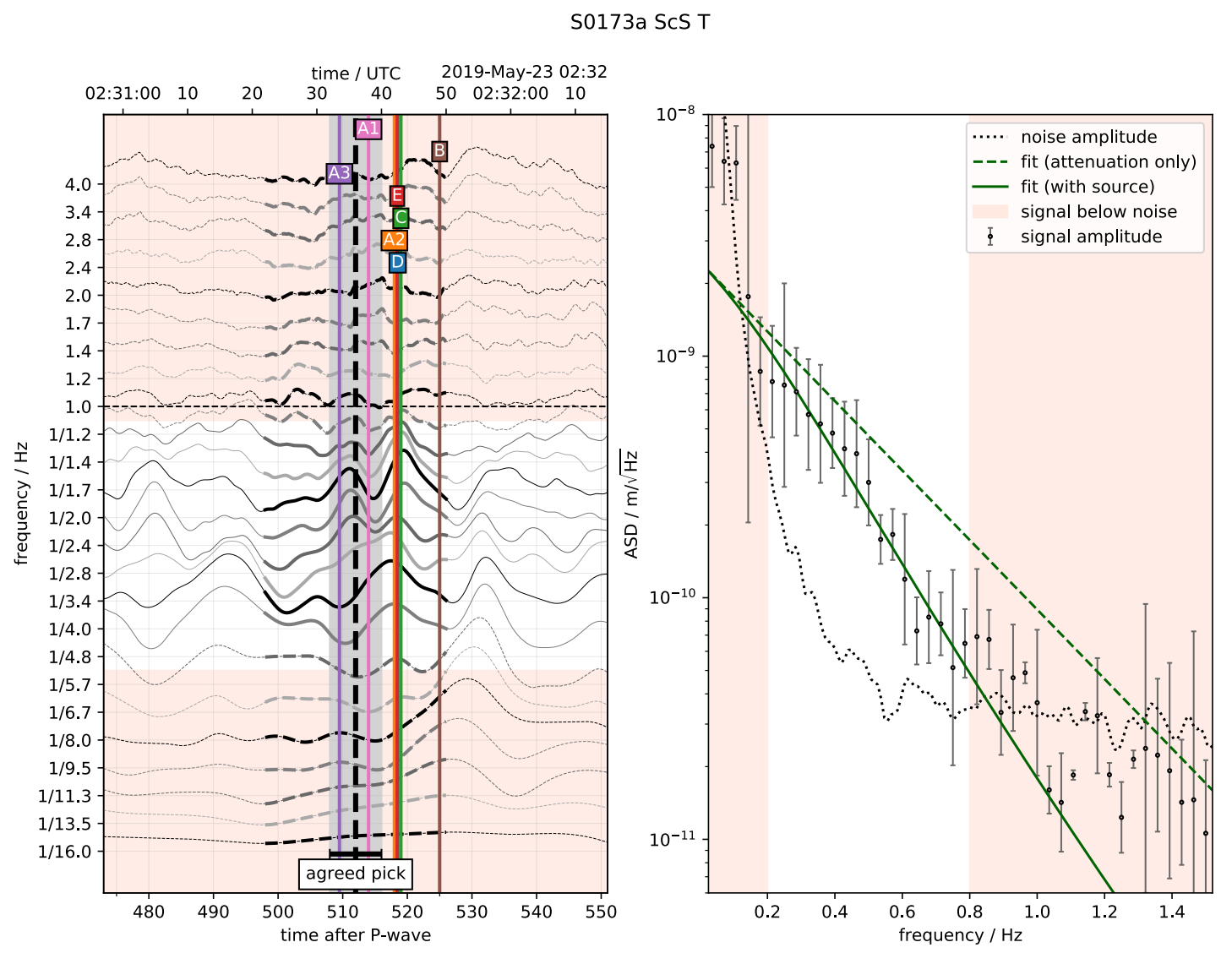

Fig. S6-6. Filter bank for S0173a - ScS-phase

The picks done by all methods (but B) clearly focus on two peaks between 510 and 520s after P. Since they both have the same spectral character, we interpret the first as ScS at $512 \mathrm{sec}$ and its depth phase sScS at $519 \mathrm{sec}$, with an uncertainty of 3 seconds. 
Submitted Manuscript: Confidential

Template revised February 2021

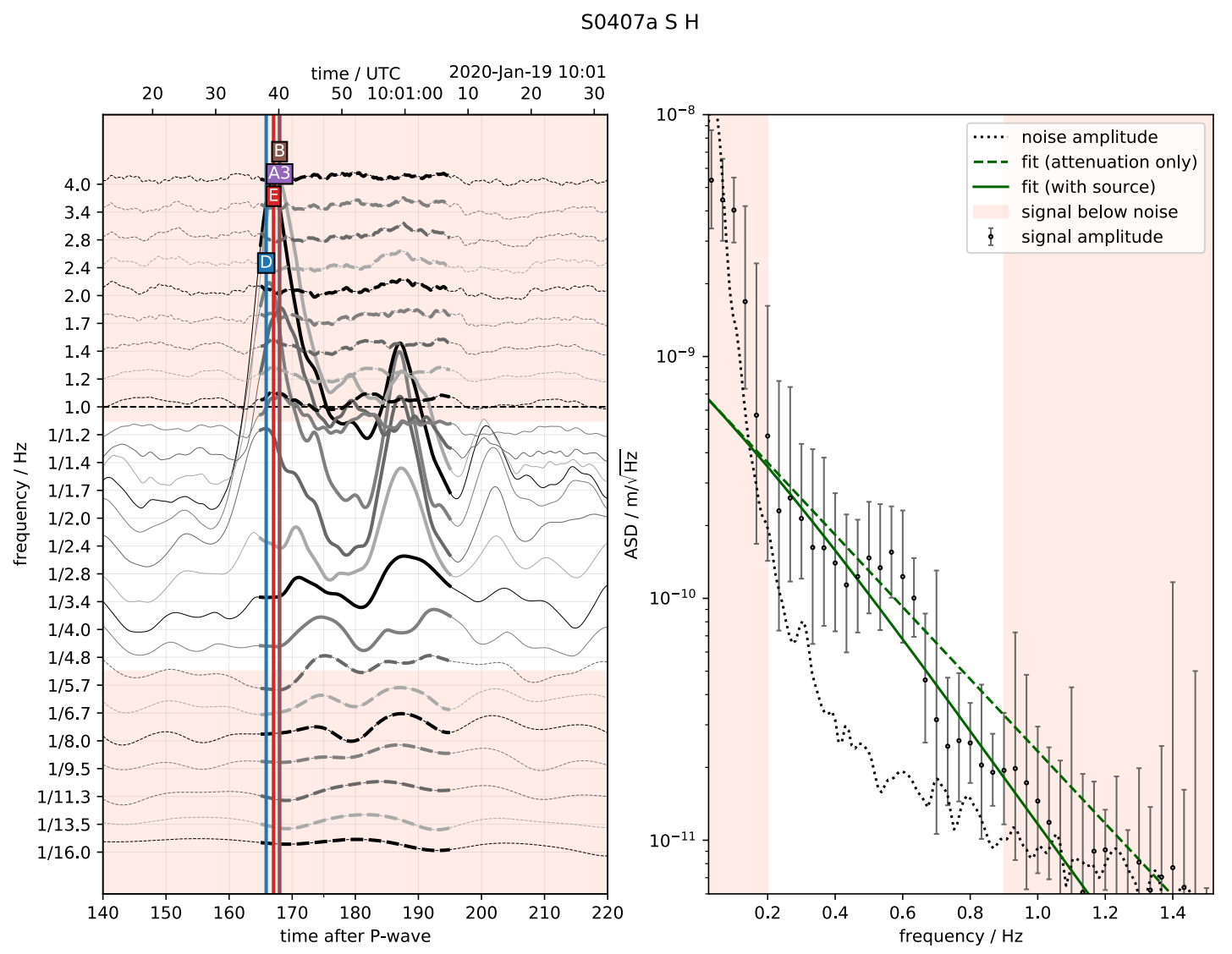

Fig. S6-7. Filter bank for S0407a - S-phase

This pick is consistent between the different methods, with very clear secondary peaks, interpreted as SS and SSS in previous studies (32). 

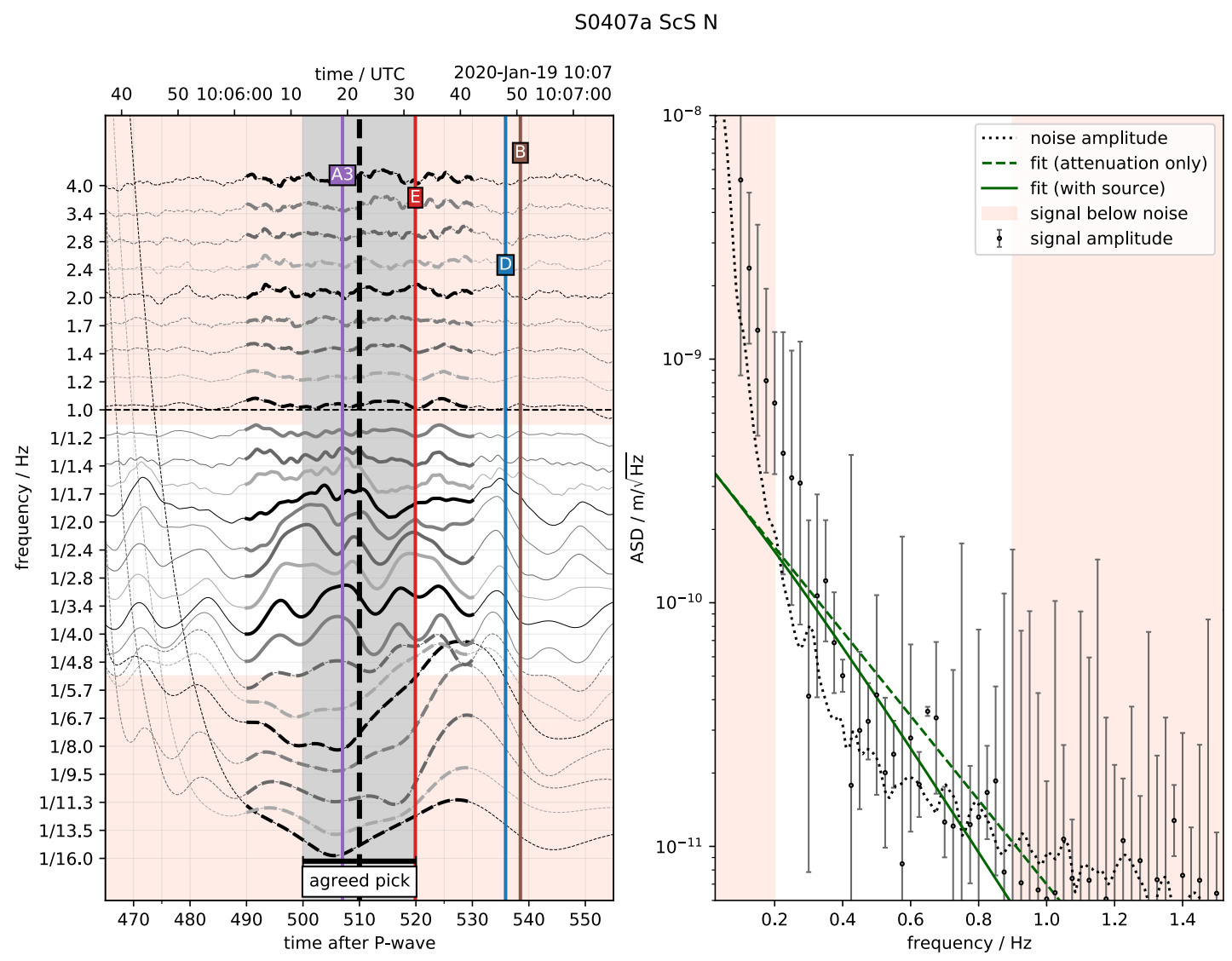

Fig. S6-8. Filter bank for S0407a - ScS-phase

The ScS picks are spread over a 30-second-long arrival around 490-520 seconds after P. We interpret the peak between 1.7 and 2.4 second period at 510 seconds as the actual arrival, with an uncertainty of 10 seconds. The sScS depth phase is uncertain, potentially at 525 seconds. 
Submitted Manuscript: Confidential

Template revised February 2021

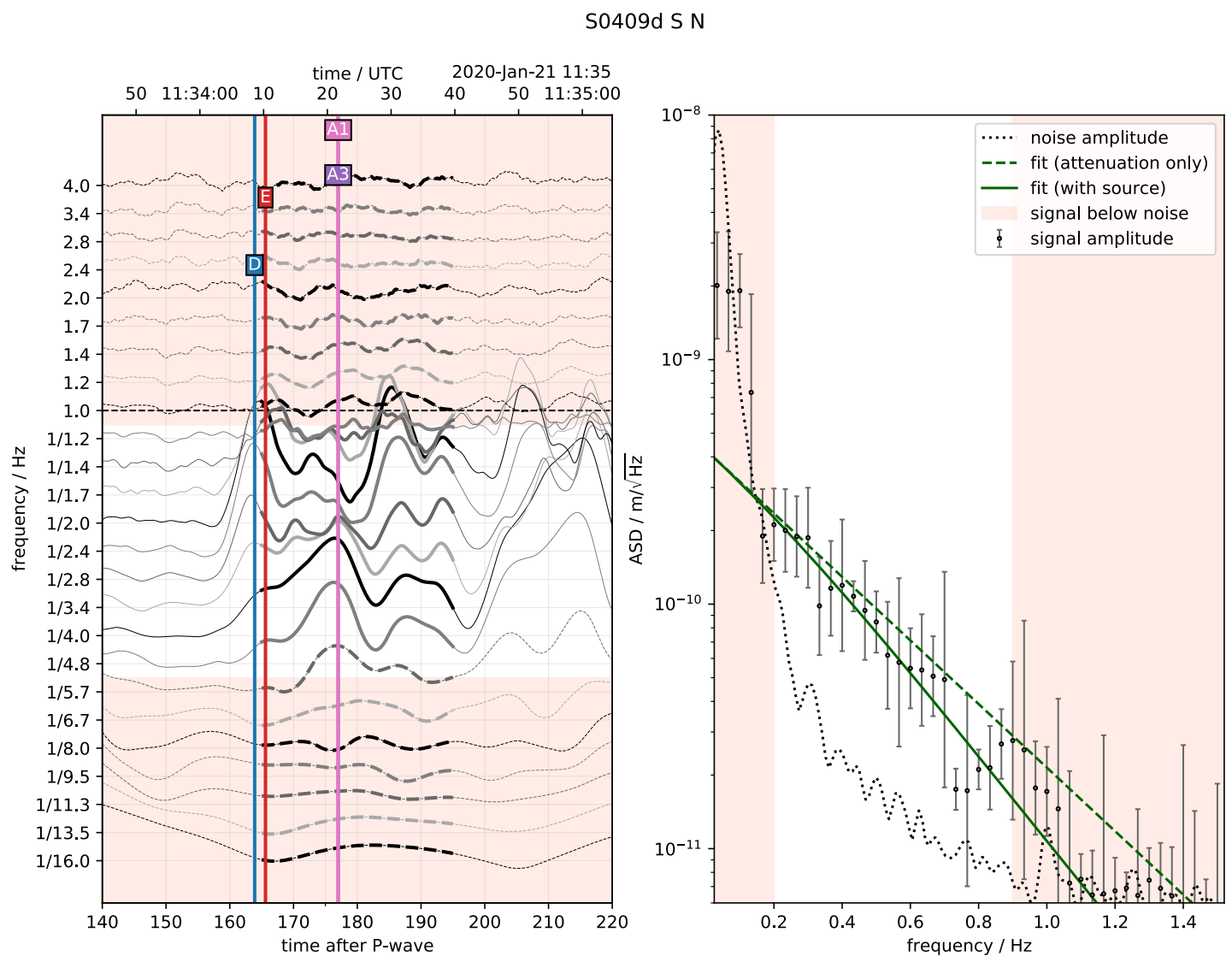

Fig. S6-9. Filter bank for S0409d - S-phase

This pick is consistent, apart from the late picks with method A. 


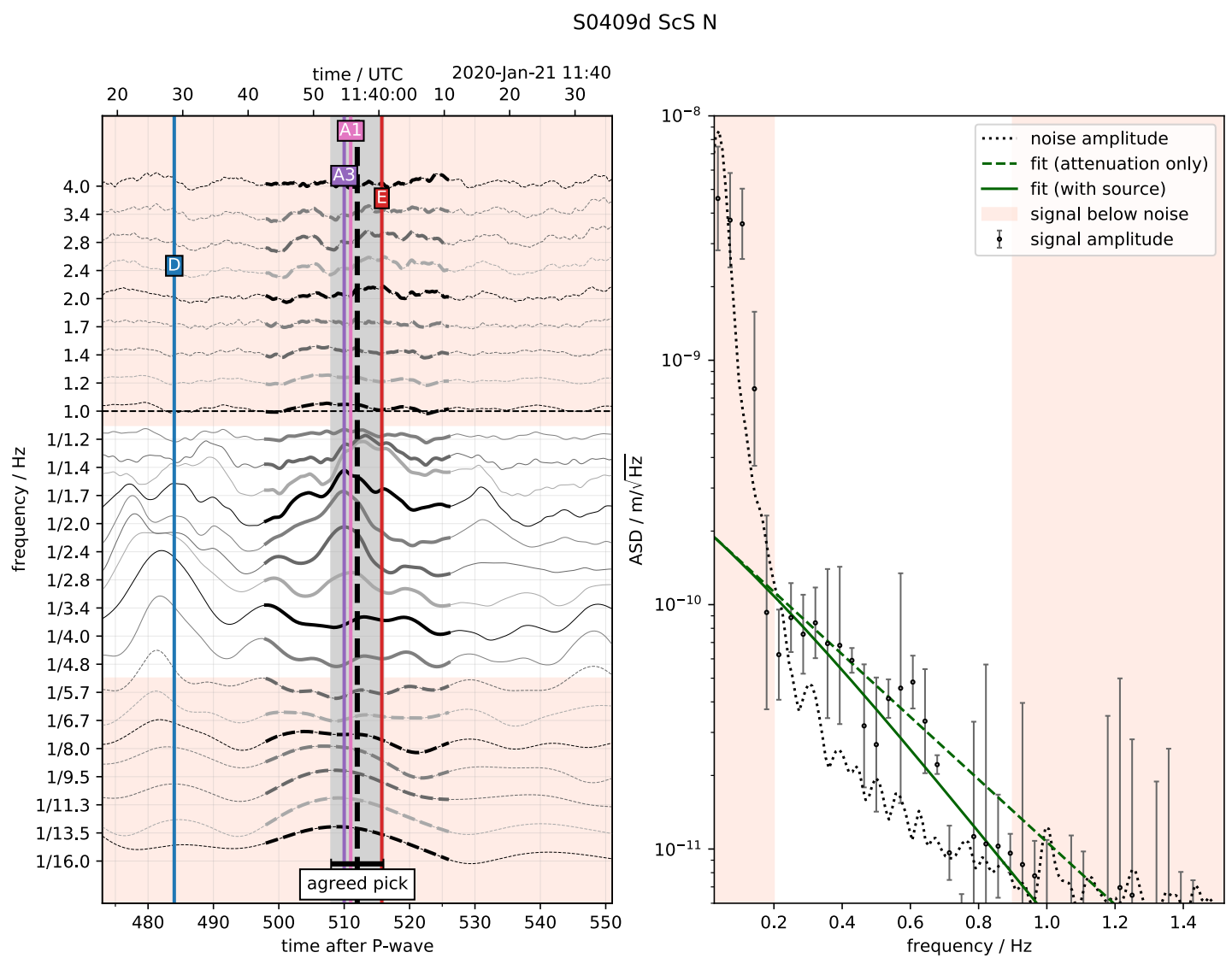

\section{Fig. S6-10. Filter bank for S0409d - ScS-phase}

The ScS pick is associated with a 10-second-long peak around 507-517 seconds after $\mathrm{P}$. We interpret the peak between 1.7 and 2.4 second period as the actual arrival and pick the peak at 510 seconds with an uncertainty of 5 seconds. 
Submitted Manuscript: Confidential

Template revised February 2021

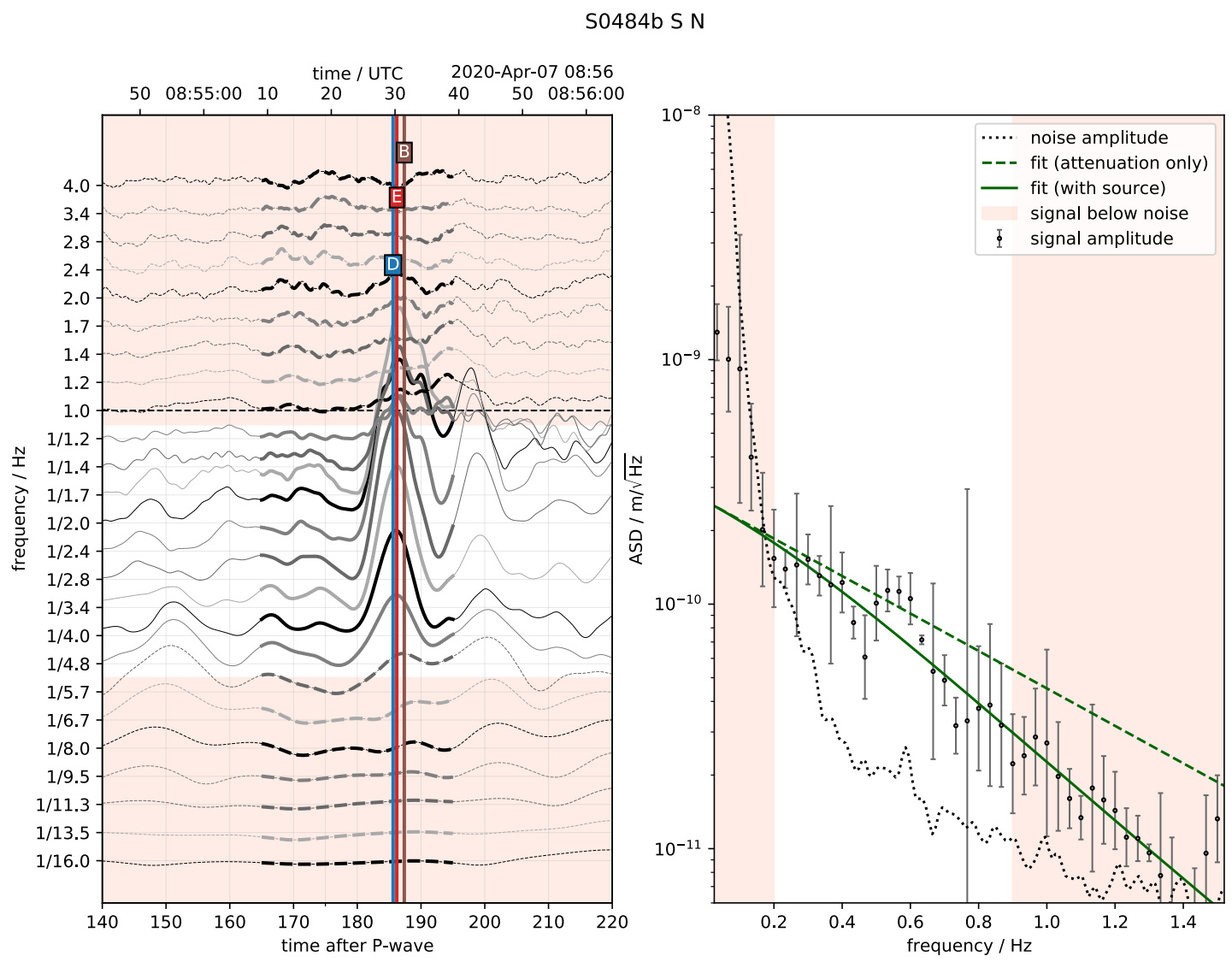

Fig. S6-11. Filter bank for S0484b - S-phase

The different methods agree on the appropriate pick. 


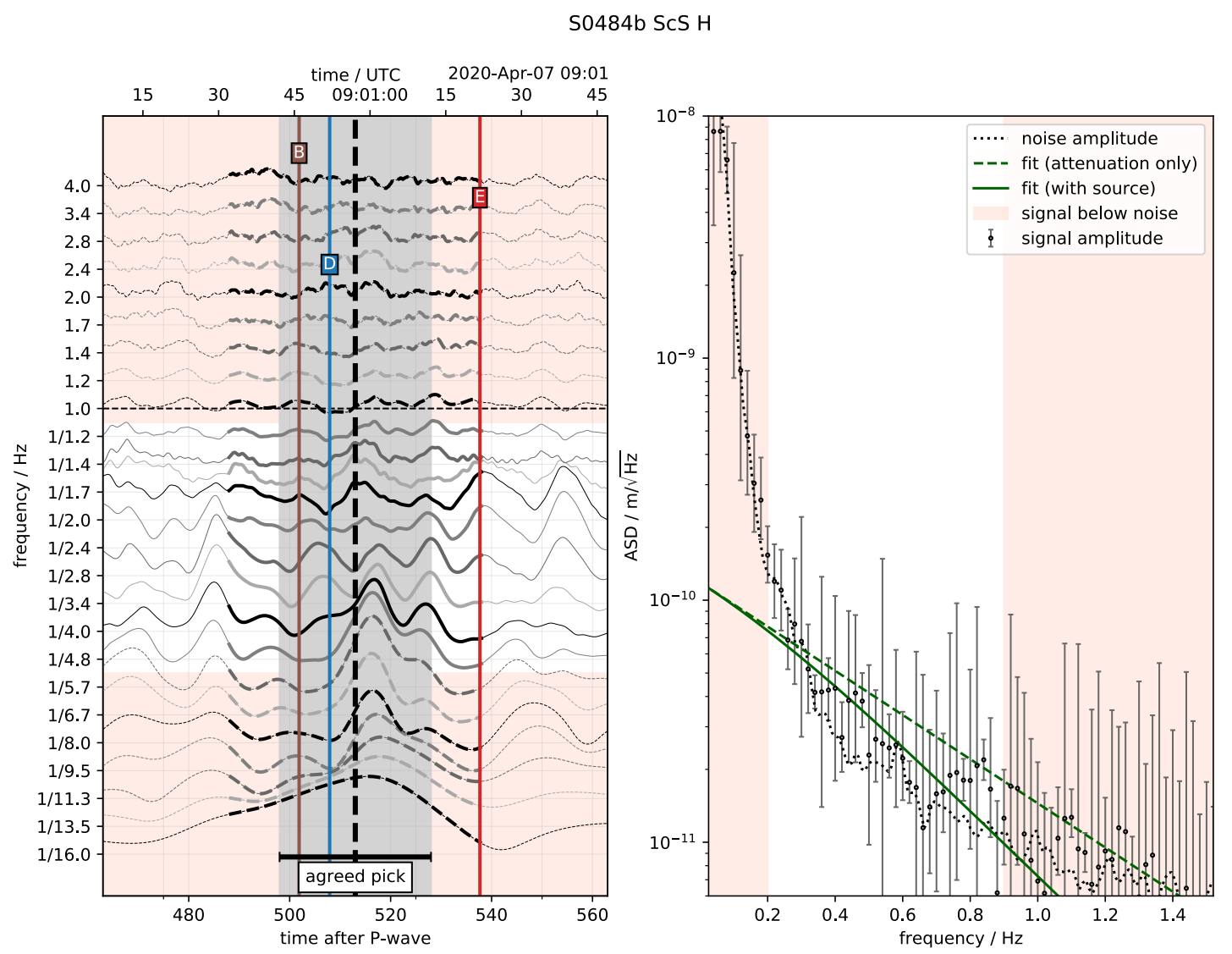

\section{Fig. S6-12. Filter bank for S0484b - ScS-phase}

While there is a signal with glitch-signature at 517 seconds, intermediate frequency energy arrives beforehand, even if that is not where the picks are made. We assign a pick at 513 seconds with a large uncertainty of 20 seconds. No depth phase is obvious. Also note that the signal is barely above the pre-event noise level. 
Submitted Manuscript: Confidential

Template revised February 2021

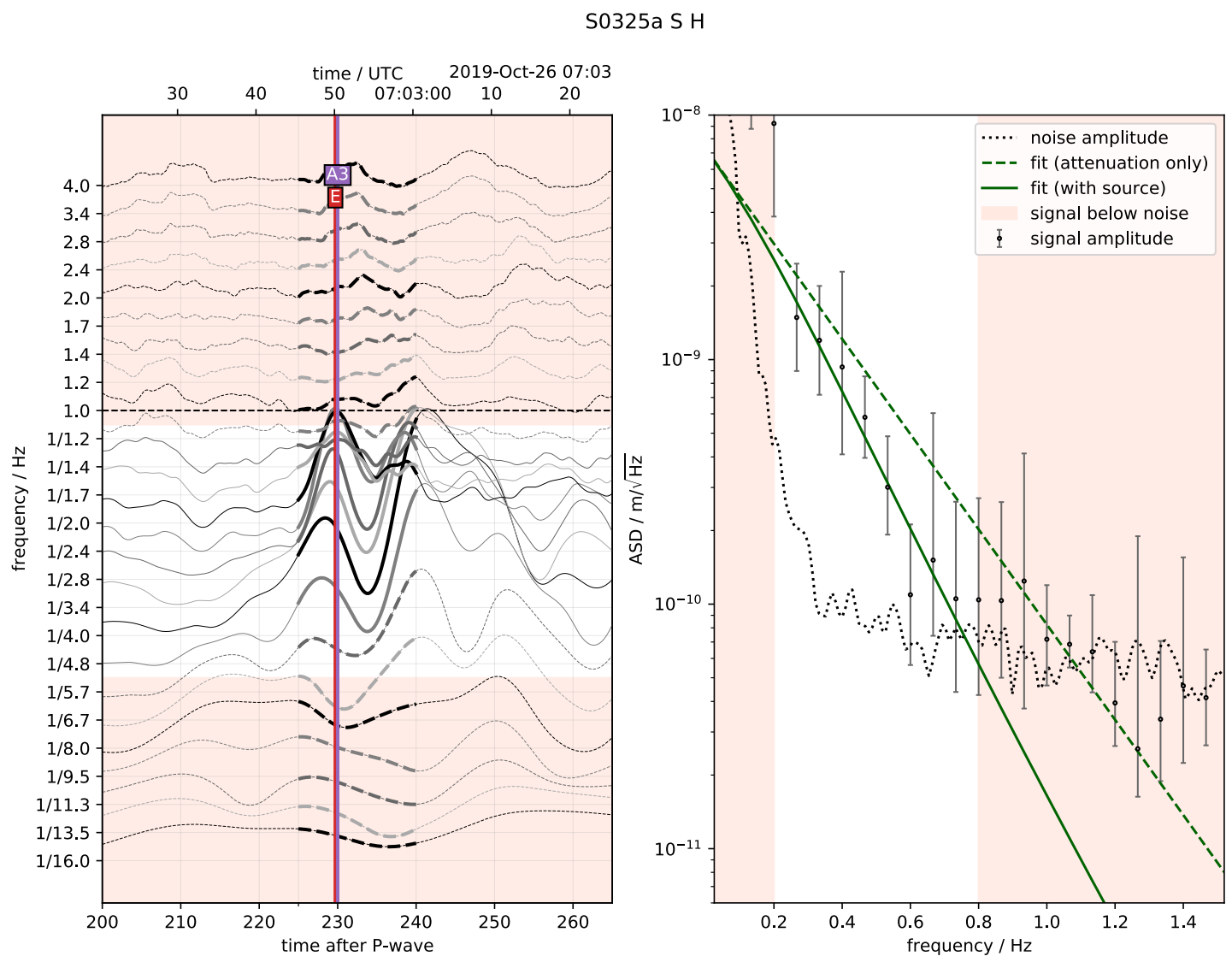

Fig. S6-13. Filter bank for S0325a - S-phase

This pick is consistent between the different methods. 


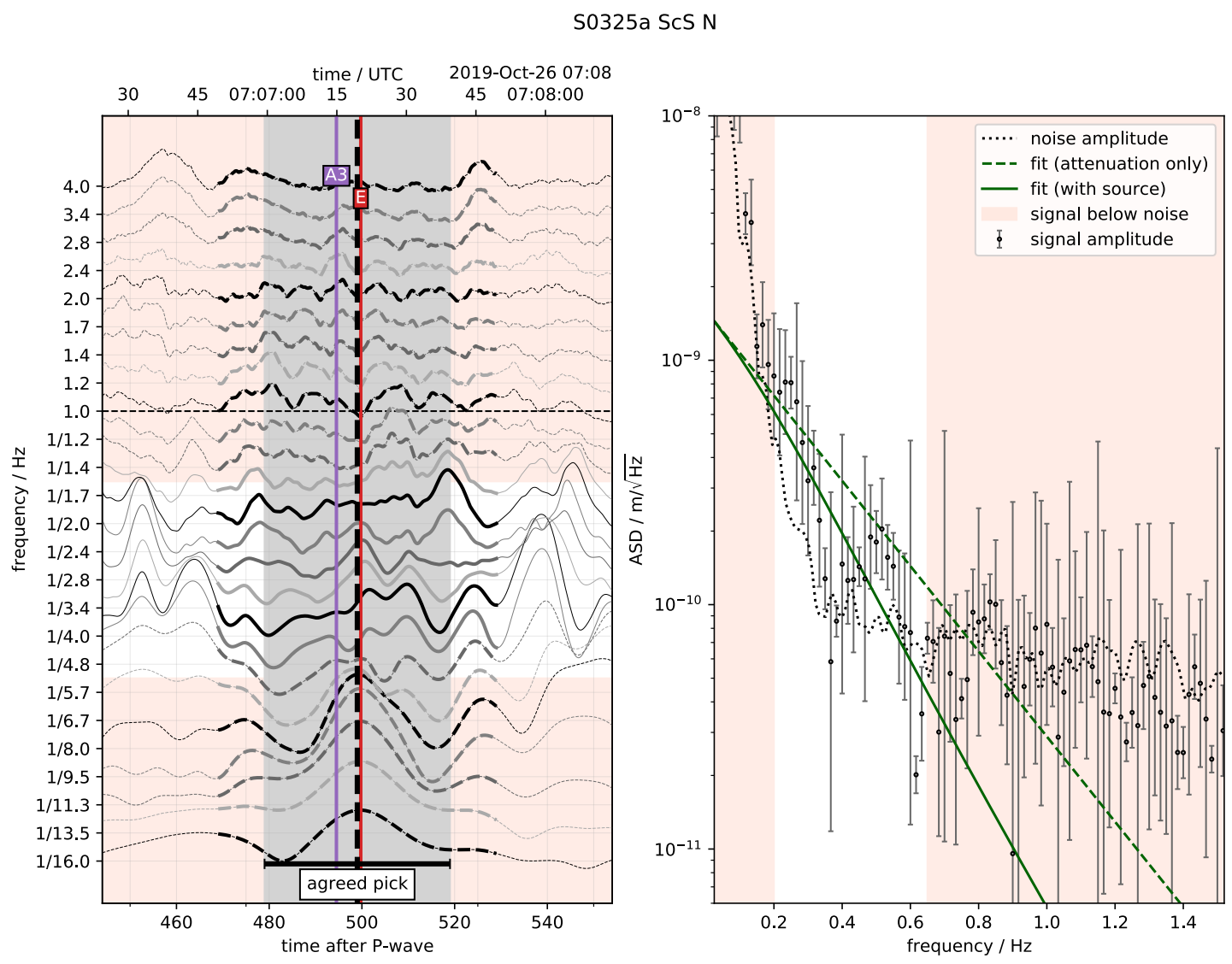

\section{Fig. S6-14. Filter bank for S0325a - ScS-phase}

The ScS pick for S0325a is inconclusive. A number of pulses arrive in a time window consistent with $\mathrm{ScS}$, as seen by the events in $25-30^{\circ}$ distance, but none of them stands out particularly. The polarization-based pick A3 extends to frequencies above $2 \mathrm{~Hz}$, which suggests wind pollution. We therefore use the vespagram based pick D at $500 \mathrm{~s}$, but with a large uncertainty of 20 seconds. 


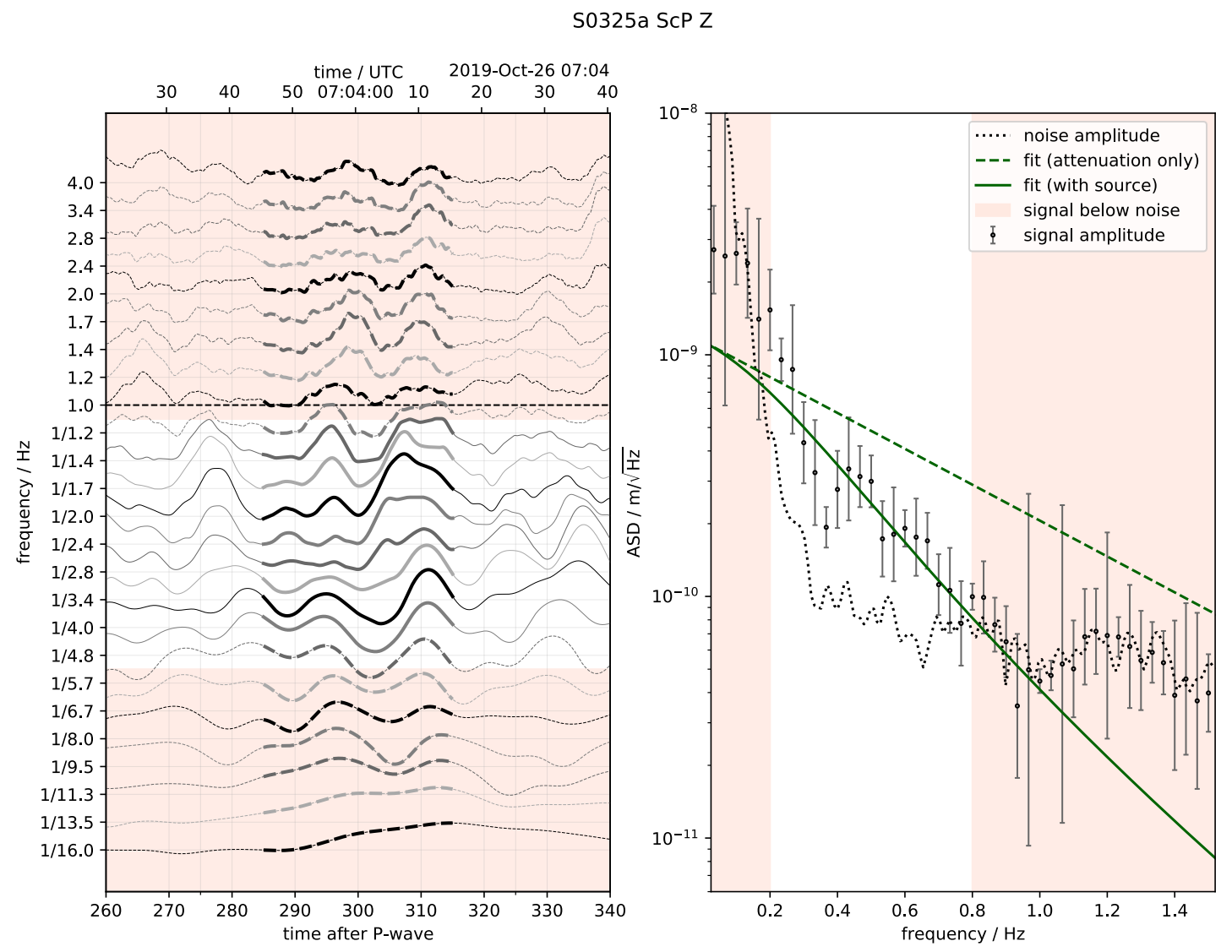

\section{Fig. S6-15. Filter bank for S0325a - ScP-phase - not used}

295 seconds after P, a signal starts with a first pulse, and a second pulse at 305 seconds. As shown by coda correlation analysis, this pulse resembles the S-wave pulse and is dominantly vertically polarized. However, the pick is right in the $\mathrm{S}$ coda and very close to an arrival which had been identified as SSS by (32), so we just note that there is a chance of observing ScP, which has to be confirmed by future quakes. 


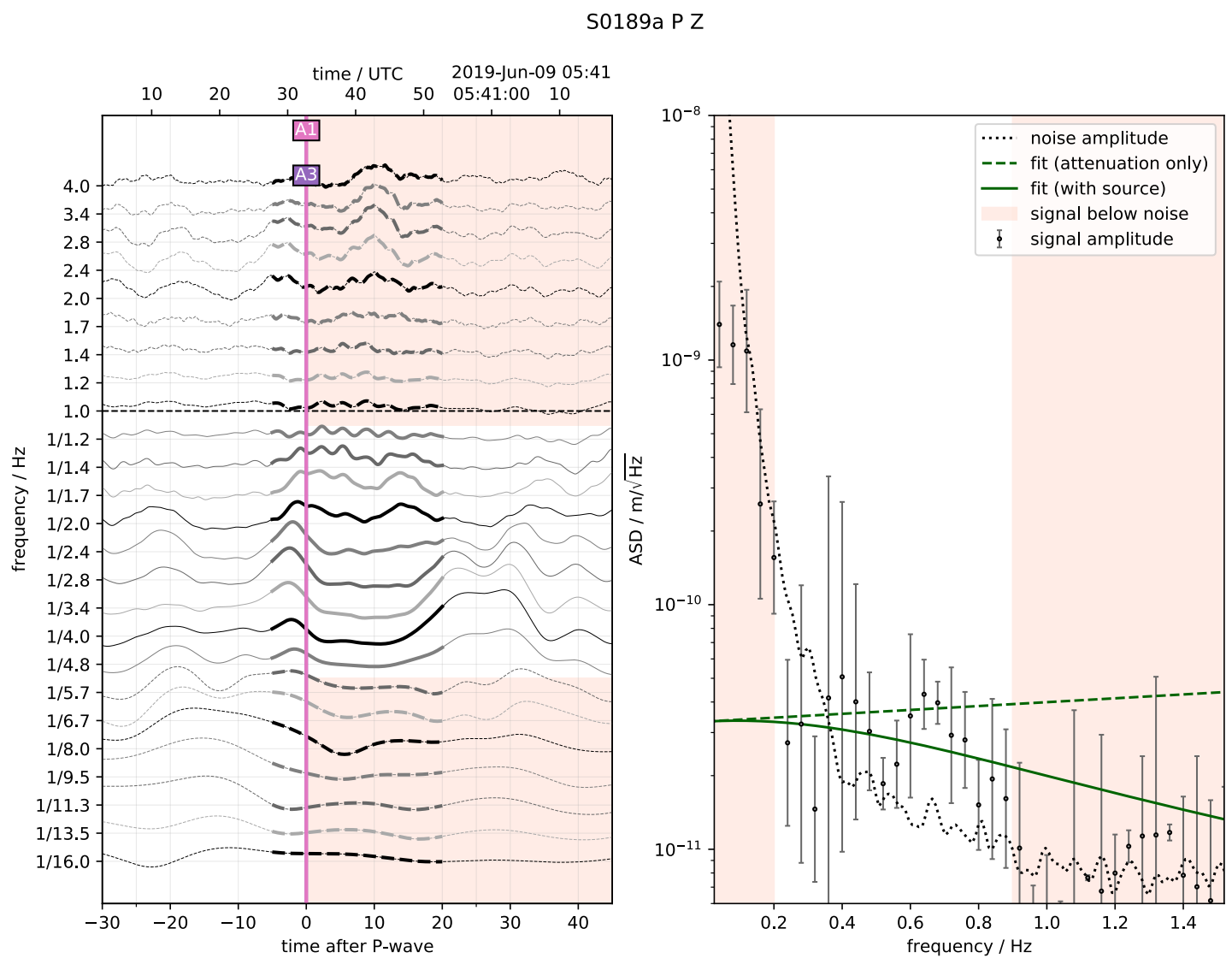

Fig. S6-16. Filter bank for S0189a - P-phase - not used

A characteristic of this event is the lack of a clear P-wave arrival, apart from relatively diffuse energy between 1 and 2 second period (99). 
Submitted Manuscript: Confidential

Template revised February 2021

S0189a S H
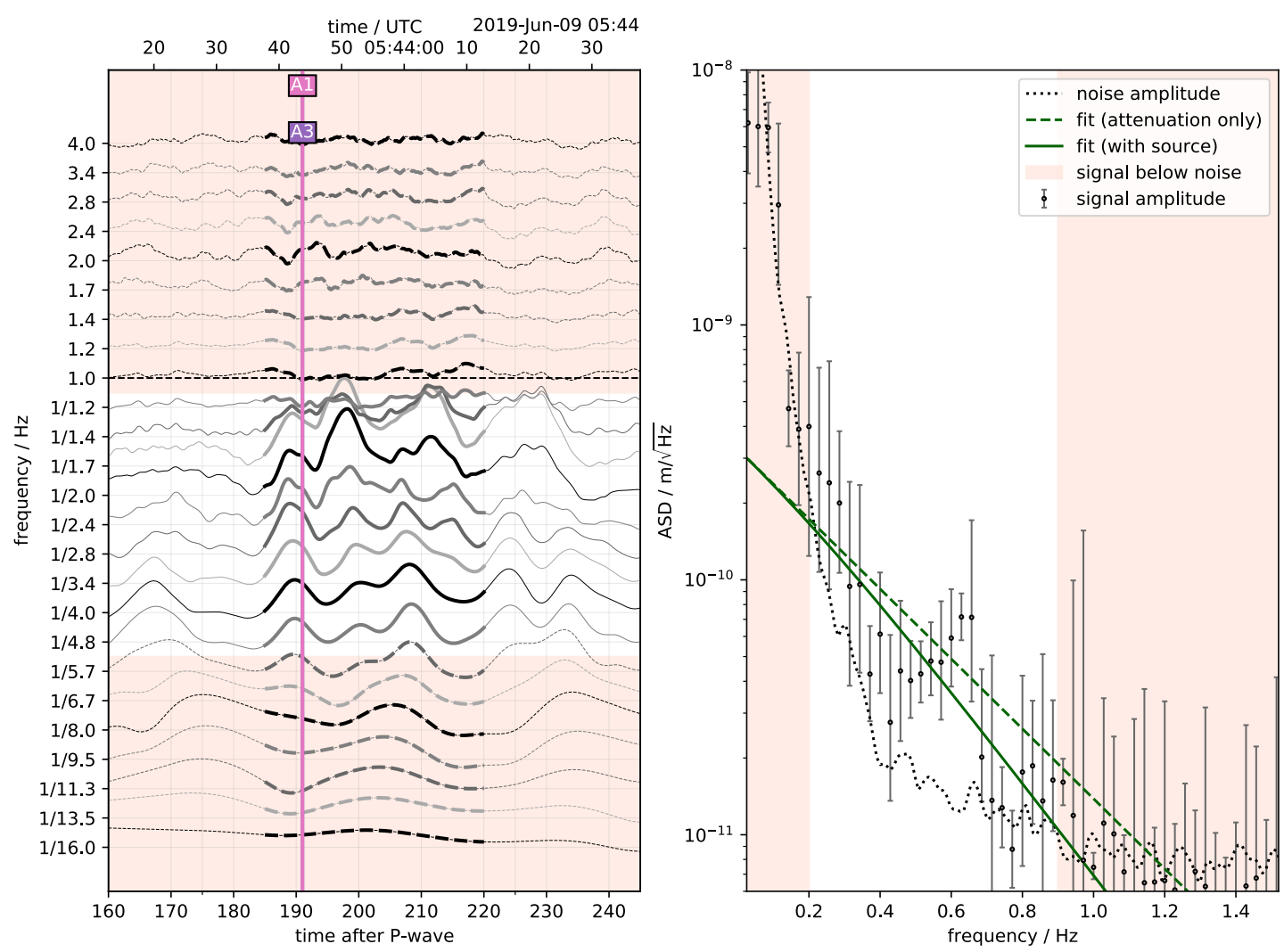

Fig. S6-17. Filter bank for S0189a - S-phase - not used

The S-wave pick is clear, but the energy ratio between direct $\mathrm{S}$ and coda is relatively low. 


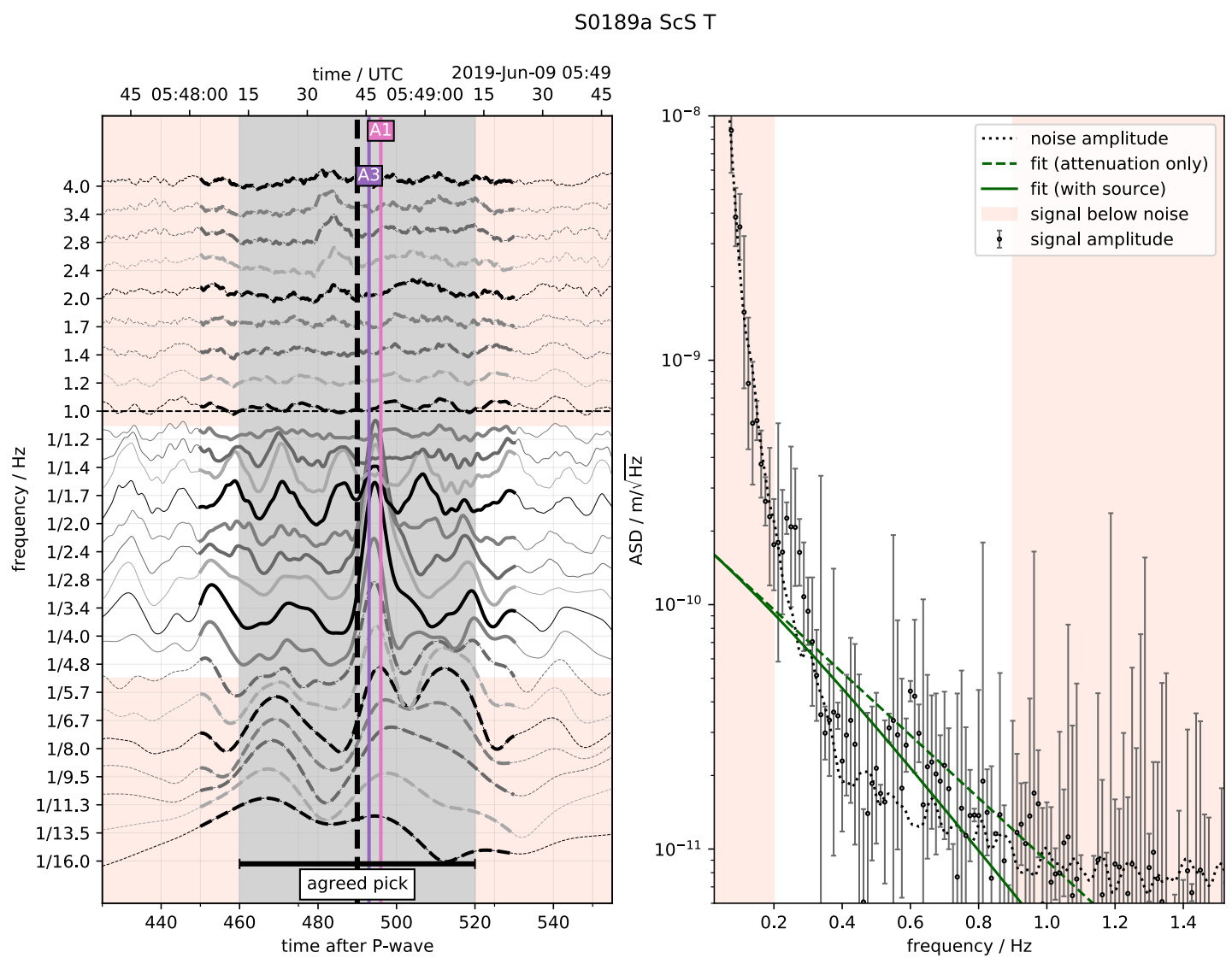

Fig. S6-18. Filter bank for S0189a - ScS-phase - not used

For this event, a strong peak between 2 and 6 seconds has been identified by two teams. The peak shows no clear long-period signature, speaking against wind pollution. Nevertheless, the lack of energy above $0.5 \mathrm{~Hz}$ is not consistent with clearer observations on other events. It is not used in the analysis. 
Submitted Manuscript: Confidential

Template revised February 2021

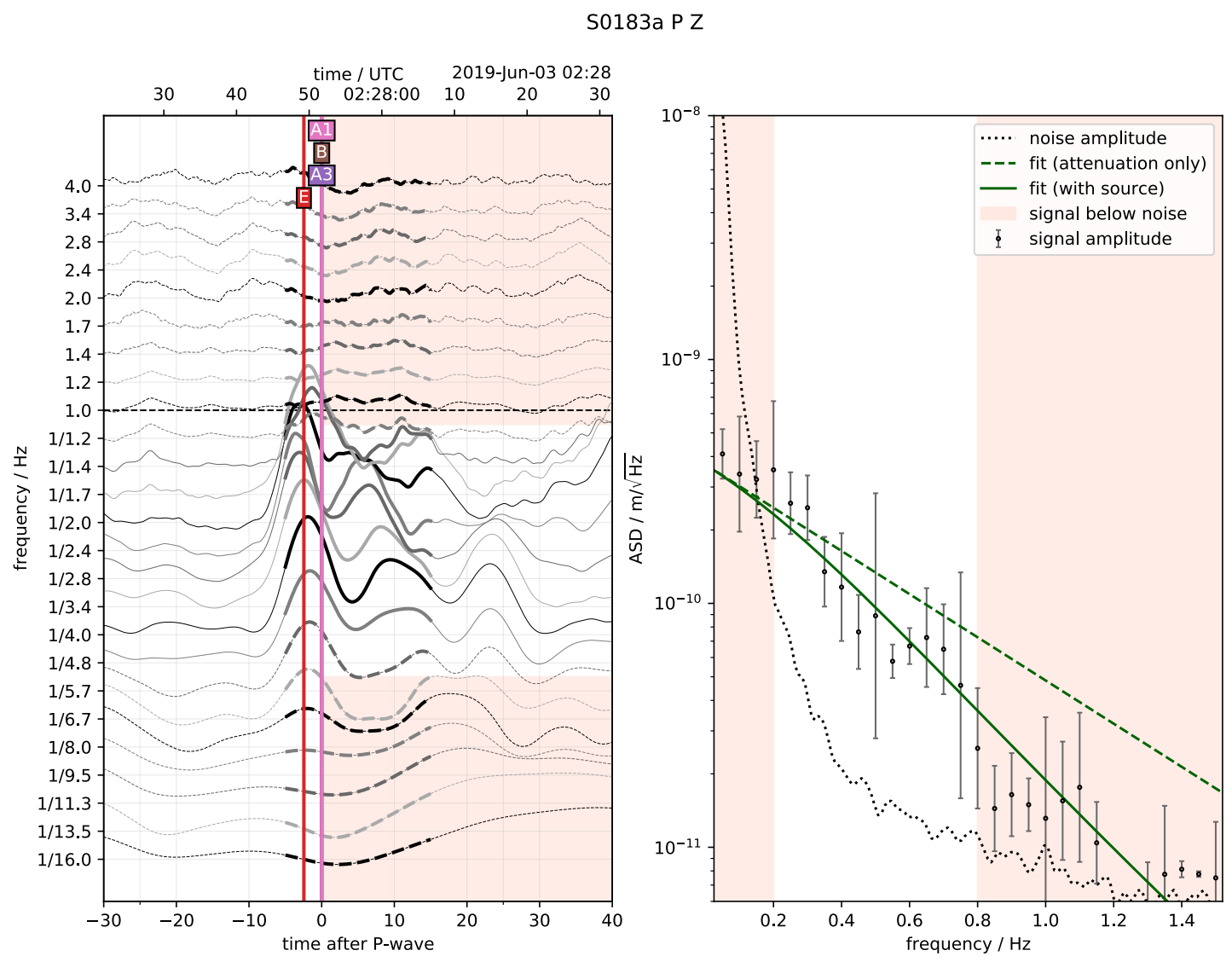

Fig. S6-19. Filter bank for S0183a - P-phase - not used

This pick is weak, but clear and allows for a clear determination of a backazimuth, see (99). 
S0183a S T
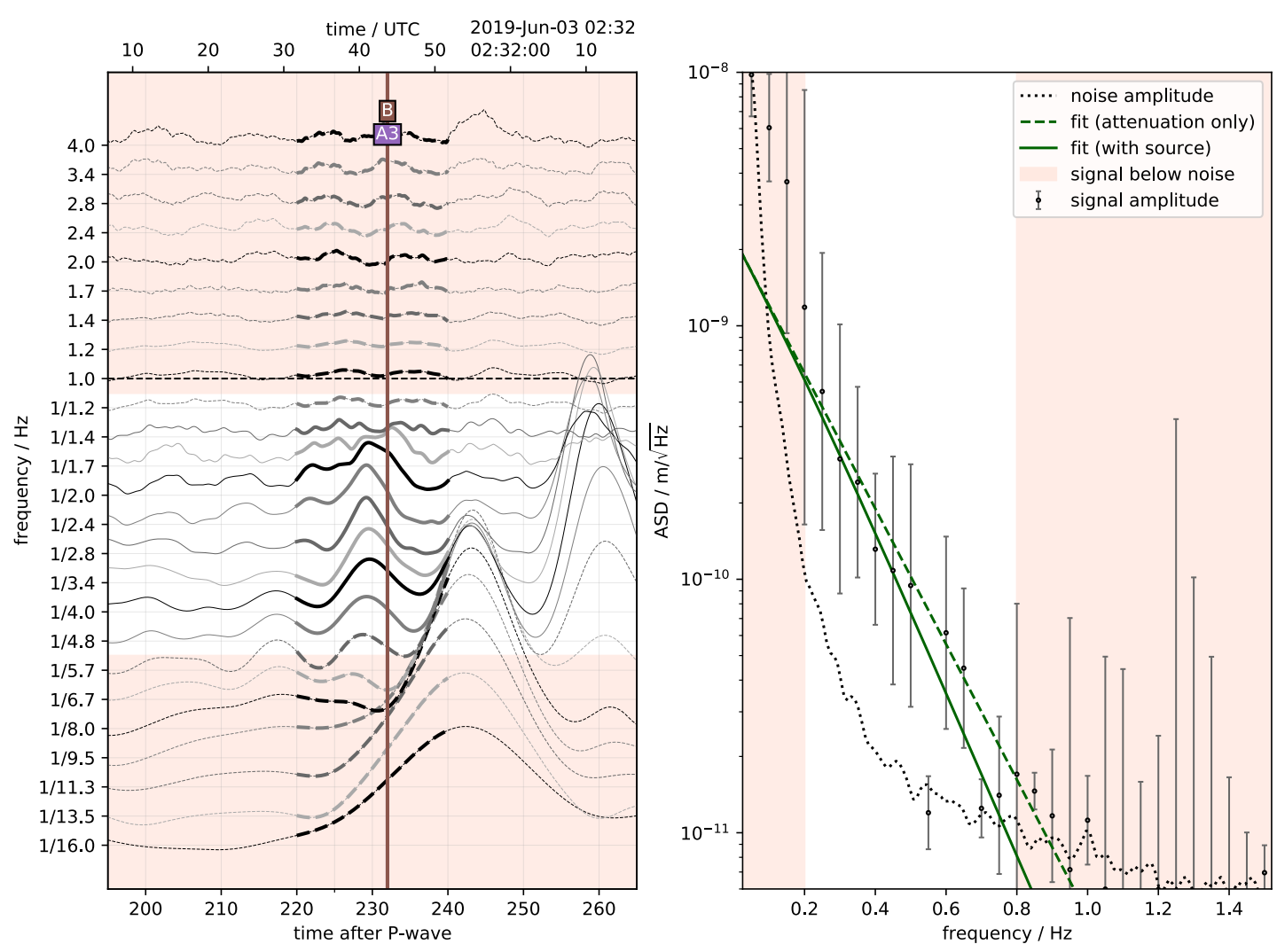

Fig. S6-20. Filter bank for S0183a - S-phase - not used

This pick is not quite clear. It is very weak, compared to all other S-arrivals and followed by a number of strong glitch-like signals. It has not been unequivocally accepted that an S-wave arrival is present in this event at all. 


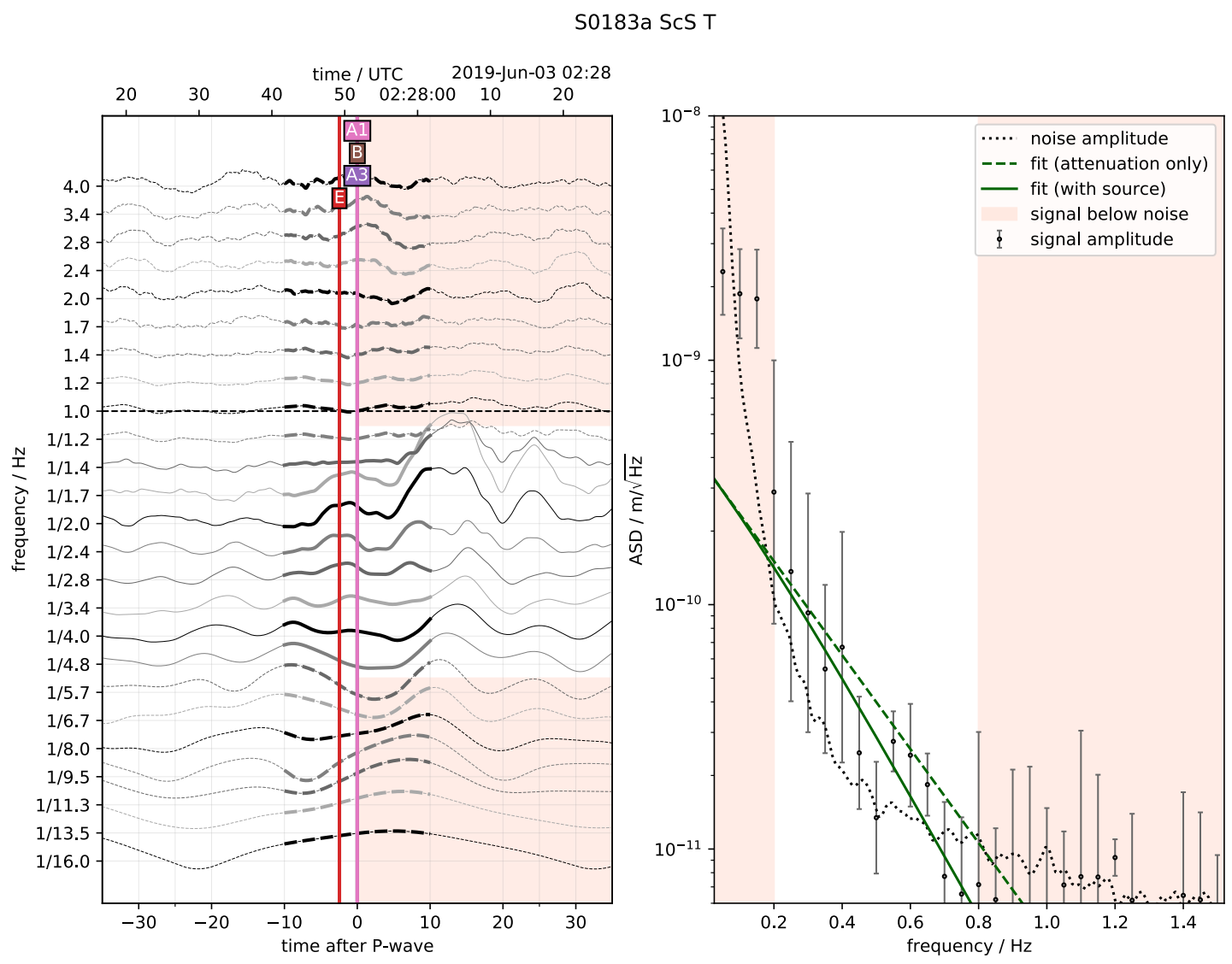

Fig. S6-21. Filter bank for S0183a - ScS-phase - not used

Assuming that the S pick is around 230 seconds, (see Fig 7-21), ScS should arrive around 460 seconds after P (if it was consistent with the less distant events). Multiple peaks are present in this time window and have been picked by different groups. An ScS pick could be assigned with large uncertainty, but given the unclear S-wave arrival, we decided against using this event in an inversion. 

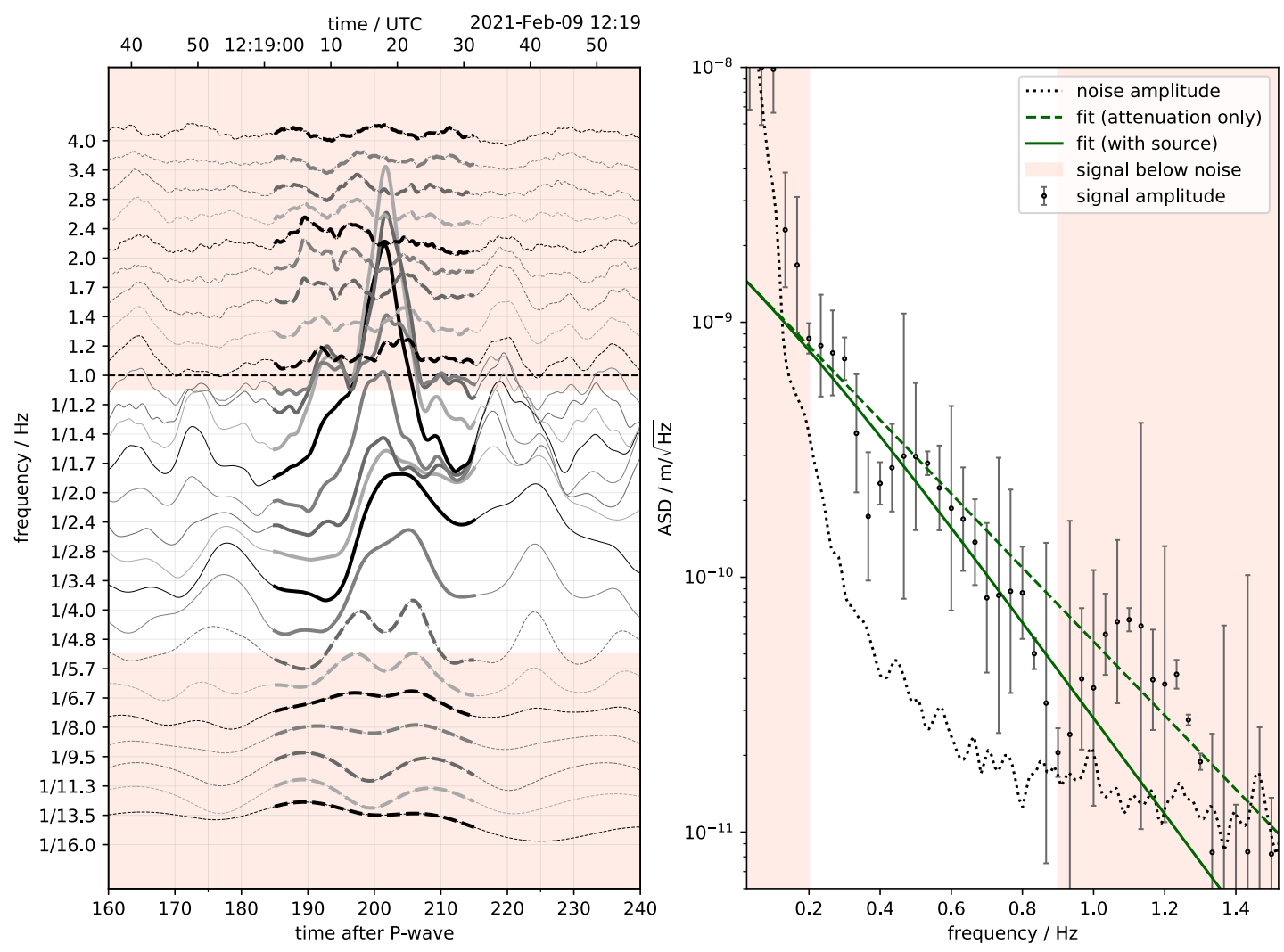

Fig. S6-22. Filter bank for S0784a - S-phase - not used

This event occurred after the first version of the analysis was done and is shown here just as a confirmation. The S-wave pick is clear. 


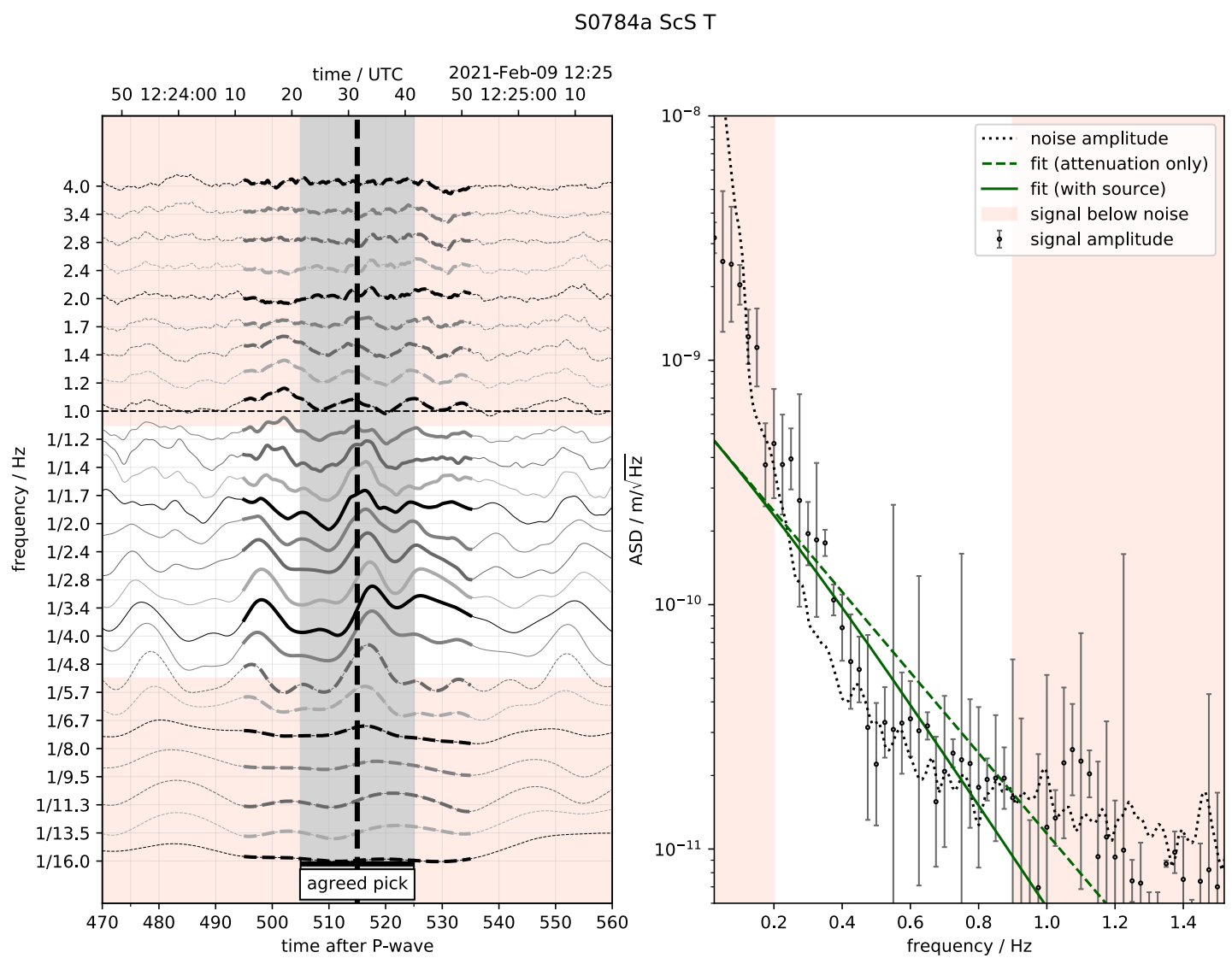

Fig. S6-23. Filter bank for S0784a - ScS-phase - not used

As written above, this marsquake occurred after the first analysis was done and was not used in the inversion. It shows a peak at 515 seconds after $\mathrm{P}$, which is consistent with the value obtained for events in similar distance, confirming the presence of a signal in the other events. 
Submitted Manuscript: Confidential

Template revised February 2021 


\section{Significance of observed phases with respect to seismic noise}

Most of the ScS phases have low SNR due to the low magnitude of the events and the observed ratio between the $\mathrm{S}$ and $\mathrm{ScS}$ amplitude, which is about 10. In this section, we analyze the robustness of the $\mathrm{ScS}$ phases with respect to the SEIS VBB self-noise, environmental seismic noise (mostly due to Martian winds) or random waves in each event's coda. We also assess the $\mathrm{SNR}$ of the ScS phases using a comodulation-based approach. We conclude that only the ScS phases of S0173a and S0235b have an SNR larger than 3 over their full bandwidth $(0.3-0.7 \mathrm{~Hz})$, enabling complete phase analyzes. The ScS phases of the other events are more embedded in the environmental noise suggesting they should mostly be used mostly in stacked analyses or in narrower frequency bandwidths.

We detail our analysis about the robustness of the ScS phases for several events in the following. First, we introduce the calculation of the SEIS VBB self-noise ranges. ScS arrivals have been mainly sought on the North component due to the backazimuth of the marsquakes. To assess the VBB self-noise in a statistical way, we model the noise on the VBB North component by assuming pink noise on the VBB U/V/W channels (28), and then calculate the amplitude spectrum on the re-oriented $\mathrm{N}$ axis. By repeating the process 1000 times, each with a different noise realization, we get a statistical model of the self-noise amplitude spectra, which will be used on the estimated self-noise amplitude and normalize the amplitudes by the mean value at each frequency (values for $0.5 \mathrm{~Hz}$ are shown in Fig. S7-1). We pick the relative amplitude ratios corresponding to the $98 \%$ of the distributions; then multiply the ratios to the VBB northcomponent instrument noise at each frequency between 0.1 and $1 \mathrm{~Hz}$. The multiplication results represent $98 \%$ of the VBB self-noise (Fig. S7-2). We also pick the $2 \%$ of the distributions (Fig. $\mathrm{S} 7-2$ ) and plot the $2 \%$ of the VBB self-noise.

We also adopt a comodulation-based SNR measurement approach as described in (110) to further assess the environmental independence of the $\mathrm{ScS}$ phases. Comodulation is a spectrotemporal approach that quantifies the correlation in signal power between the locally-measured environmental variables (wind and pressure) and ground motion, and has been demonstrated particularly successful in identifying the seismic energy that is in excess of the local background weather $(99,103,110)$. The amount of divergence between the seismic energy diverging and the expected value given the wind and pressure can then be interpreted as being from a seismic source and provides an independent set of signal-to-environmental-noise SNR values.

\section{S0173a:}

Robustness analysis method and description of results: We start with the $\mathrm{ScS}$ candidate phase of S0173a. We use the transverse (T) component data for the event (backazimuth $91 \mathrm{deg}$, (99)). Based on the polarization analysis and waveform correlation with $\mathrm{S}$ waves, we find that the $\mathrm{ScS}$ phase is in a $10 \mathrm{~s}$ time window starting at UTC 2019-05-23T02:31:39. The ScS amplitude spectrum is much larger than the noise. The noise that is present can be primarily attributed to environmental factors because it is substantially larger than the $98 \%$ VBB self-noise between 0.1 and $0.9 \mathrm{~Hz}$ (Fig. S7-2b), leaving the environment as the primary source of noise for that phase. Martian environmental noise is mainly caused by winds, and the square of the wind speed is positively correlated with the seismic amplitudes (31). Thus, we check the wind speed data in one minute around the $\mathrm{ScS}$ window and for same local time (i.e., Martian hour/minute/second) but on 10 surrounding Sols nearby (from Sol 168 to 178 excluding Sol $173)$; we also search for similar wind speeds $( \pm 0.25 \mathrm{~m} / \mathrm{s})$ in 2 hours centered around the $\mathrm{ScS}$ 
time window on these 10 Sols. We plot those wind-speed data into distributions for wind speed squares (Fig. S7-2a). We also project those distributions to the S0173a T direction. Different wind directions on the 10 Sols make the 10 Sol distributions wider on the $\mathrm{T}$ direction than before the projection.

We then compute the T-component seismic noise in these time windows and present the associated cumulative statistics. This is done for the statistics corresponding to the same local time in the 10 surrounding Sols (Fig. S7-2b) and for the similar squared wind speeds too (Fig. S7-2c). Last but not least, we estimate ground velocity spectral amplitudes due to winds using the relation proposed by $(29)$, detailed in $(93,111)$, and then plot the estimates for the wind values at the time of the $\mathrm{ScS}$ observation. All these analyses show that the amplitude of the $\mathrm{ScS}$ is about 3-5x larger between 0.3 and $0.6 \mathrm{~Hz}$ (Fig. S7-2b and 2c) than these estimations of noise from wind and is therefore unlikely to be associated with wind related environmental effects.

We finally compare the ScS amplitude to the coda. We choose ten windows from 100s to 200 s after the $\mathrm{ScS}$ and use these 10 window recordings as coda noise near the ScS. This coda noise (shown in gray in Fig. S7-2b-d) has larger amplitude than the environmental noise analyzed earlier. If the $\mathrm{ScS}$ candidate phase (plotted in red) was a random phase from the coda, it shall have a similar amplitude as the coda noise. The $\mathrm{ScS}$ spectral amplitude is however about 2.5 times larger than the coda amplitudes at $0.4 \mathrm{~Hz}$. We therefore conclude that the S0173a ScS is very significantly above the environmental and coda noise of the record.

Comodulation analysis method and description of results: The comodulation approach adopted from (110) uses the method of moments to match the mean and variance of the seismic and environmental signals prior to the start time of the putative marsquake. We obtain the first two moments for all signals five minutes prior to the marsquake event and ignore any identified glitches in the record which could contaminate these statistics. The method of moments is then used to match the mean and variance of the seismic acceleration and pressure root mean square (RMS) envelopes to the measured wind speed signal. The seismic RMS envelope is estimated as the total power of all three combined Z, N and E components. Both seismic and pressure RMS envelopes are extracted in discrete half-octave frequency bands from $1 / 32 \mathrm{~Hz}$ up to the anti-alias filter $8 \mathrm{~Hz}$ of the continuous 20 sample-per-second (sps) VBB data. We then estimate respective $\mathrm{SNR}_{\mathrm{w}}$ (wind) and $\mathrm{SNR}_{\mathrm{p}}$ (pressure) values over various frequency bands quantifying the independence of the seismic signal from the expected noise from environmental injection for the time window beginning at the start of the $\mathrm{ScS}$ phase and up to 60 seconds later.

The comodulation-based approach is demonstrated in Fig. S7-3 for the S0173a event. The spectrogram of the S0173a event is shown at the top panel of Fig. S7-3a, while the bottom panel shows the matched-moment seismic and environmental signals over the event's duration. The $\mathrm{ScS}$ phase is shown by the black dotted line. Computed mean $\mathrm{SNR}_{\mathrm{w}}$ and $\mathrm{SNR}_{\mathrm{p}}$ values over discrete frequency bands are shown in Fig. S7-3b, with an SNR value $>3$ against both local pressure and wind noise injection in the frequency band $0.25-0.5 \mathrm{~Hz}$, consistent with the robustness analysis.

\section{$\underline{\text { S0235b }}$}

Robustness analysis results: We then apply the above analysis to S0235b transverse component (backazimuth $73 \mathrm{deg}$, (99)), with a corresponding ScS starting at UTC 2019-0726T12:27:49 (Fig. S7-4). We observe that this ScS also possesses over 98\% possibility for being real ground motions between 0.1 and $0.8 \mathrm{~Hz}$. Note however that we have a gap in wind speed data from 12:25 to 12:35, therefore the wind noise cannot be estimated from wind records as previously. We observe nevertheless that the S0235b ScS amplitudes are about 5 times larger 
than those recorded at the same local time the 10 closest sols and 2 times larger than the coda amplitudes around $0.3 \mathrm{~Hz}$ (Fig. S7-4c).

Comodulation-based analysis results: We also apply the comodulation approach to evaluate the independence of the S0235b event and its ScS phase to environmentally-induced local noise effects. While there is a gap in the wind-speed data preventing us from obtaining an $\mathrm{SNR}_{\mathrm{w}}$ of seismic to wind-noise effects, Fig. S7-3b indicates that the $\mathrm{SNR}_{\mathrm{p}}$ to pressure-induced noise effects exceeds 20 in the frequency range of $0.25-0.7 \mathrm{~Hz}$, while $\mathrm{SNR}_{\mathrm{p}}$ is also greater than 5 in the frequency range $1 / 5.7-1 \mathrm{~Hz}$. Notice that, due to the lack of the wind speed data, this analysis is not as complete as for other events.

All other events, because of their smaller amplitudes, will have much lower signal to noise for the $\mathrm{ScS}$ window. Due to the larger uncertainties, we increase the seismic time windows for these events from 10 s to 20 s.

\section{S0325a:}

Robustness analysis results: For S0325a (Fig. S7-5), we observe that $\mathrm{ScS}$ is above the $98 \%$ self-noise from 0.1 to $0.9 \mathrm{~Hz}$. However, only the ScS spectral amplitude peaks around 0.3 , 0.55 and $0.8 \mathrm{~Hz}$ are larger than $90 \%$ of the seismic noise at the same local time or under the similar wind speed (Fig. S7-5b, and S7-5c). This could be because part of the wind speeds in the surrounding 10 Sols are larger than the equivalent $\mathrm{ScS}$ amplitude. For example, the wind-speedsquare maxima on the same local time is only 1.5 times the wind-speed-maximum at the $\mathrm{ScS}$ (Fig. S7-5a). Thus, these large noise recordings in the 10 Sols are likely due to larger winds. The $\mathrm{ScS}$ amplitudes are also larger than those estimated for ground velocities with the observed wind and slightly larger than the coda at 0.55 and $0.8 \mathrm{~Hz}$. This suggests that frequency analysis of the $\mathrm{ScS}$ of S0325a might focus only at these two frequencies.

Comodulation-based analysis results: S0325a occurs in a relatively noisier period, with a signal-to-environmental-noise $\mathrm{SNR}_{\mathrm{w}}$ and $\mathrm{SNR}_{\mathrm{p}}$ values increasing for frequencies $<0.25 \mathrm{~Hz}$ due to the co-occurrence of a glitch at the ScS phase (Fig. S7-3b). The glitch does not extend beyond $0.25 \mathrm{~Hz}$, and the SNRs at the ScS phase against both pressure and wind are $\sim 1.7$ in the frequency band $0.25-0.35 \mathrm{~Hz}$, decreasing to a value of $\sim 1$ at $1 \mathrm{~Hz}$.

All data processing parameters match those in Fig. S7-2 except that the time windows increase from 10s to 20s in length. In Fig. S7-2 a) The wind speed data are from Sol 320 to 330. S0325a backazimuth is $108 \mathrm{deg}$ and ScS starts at UTC 2019-10-26T07:07:15.

\section{S0407a:}

Robustness analysis results: For S0407a (Fig. S7-6), $\mathrm{ScS}$ is above the $98 \%$ on several narrow frequency bands. The $\mathrm{ScS}$ amplitude is larger than the noise recorded at the same local time only at $0.35 \mathrm{~Hz}$ (Fig. S7-6b and S7-6c). Like earlier, this could also be due to larger-speed winds on the 10 Sols (Fig. S7-6a). The wind-speed-square maxima on the same local time are indeed about 3 times larger than the wind-speed-maximum recorded in the ScS, and two times larger when we consider the projection on the transverse azimuth. At $0.35 \mathrm{~Hz}$, the $\mathrm{ScS}$ amplitude remains also larger than the ground velocity estimate from wind speed value at the ScS time and is larger than $90 \%$ of the coda noise. This suggests limiting the $\mathrm{ScS}$ analysis to frequencies around $0.35 \mathrm{~Hz}$.

Comodulation-based analysis results: The $\mathrm{ScS}$ phase of the $\mathrm{S} 0407$ a coincides with an increase in the wind speed. Comodulation $\mathrm{SNR}_{\mathrm{w}}$ values against the wind indicate a consistent value at or below 1 for all frequency bands except below $0.1 \mathrm{~Hz}$ (Fig. S7-3b), which indicates an increase in the SNR due to the occurrence of a glitch. These $\mathrm{SNR}_{\mathrm{w}}$ values therefore indicate that 
the ScS phase for S0407a is heavily contaminated by wind-noise injection and may be embedded within the environmental noise.

\section{S0409d:}

Robustness analysis results: For S0409d (Fig. S7-7), ScS is larger than the $98 \%$ self-noise level only on two narrow frequency bands, from 0.3 to $0.45 \mathrm{~Hz}$ and from 0.55 to $0.65 \mathrm{~Hz}$. We also observe that only the $\mathrm{ScS}$ amplitudes around $0.6 \mathrm{~Hz}$ are larger than the seismic recordings at the same local time or under the similar wind speed (Fig. S7-7b and S7-7c). This could also be due to high-speed winds on the surrounding $10 \mathrm{Sols}$, as the wind speed squares at the $\mathrm{ScS}$ are about a quarter of the ones at the same local time and lower than the ones under a similar wind speeds on the transverse direction (Fig. S7-7a). This suggests an ScS SNR $>2$ mostly between 0.55 and $0.65 \mathrm{~Hz}$ (Fig. S7-7d).

Comodulation-based analysis results: While the $\mathrm{ScS}$ phase of the $\mathrm{S} 0409 \mathrm{~d}$ event shows a clear peak for both $\mathrm{SNR}_{\mathrm{w}}$ and $\mathrm{SNR}_{\mathrm{p}}$, the values are consistently at or below 2 (Fig. S7-3b). Both SNR values increase above 1.6 for the frequency bands between $0.25-0.7 \mathrm{~Hz}$ and peak to $\sim 2$ in the frequency range $0.35-0.5 \mathrm{~Hz}$.

\section{S0484b:}

Robustness analysis results: For S0484b (Fig. S7-8), the ScS amplitudes are lower than the ground velocity estimates due to the winds during the observation window. We refine the noise analysis by also using the projection of the wind speeds in the T-component direction. The $\mathrm{ScS}$ is then above that threshold in two frequency bands: $0.45-0.65 \mathrm{~Hz}$ and $0.7-0.9 \mathrm{~Hz}$. The ScS amplitudes in these two frequency bandwidths are also larger than $70 \%$ of the seismic recordings at the same local time or under the similar wind speed. We also observe that the ScS amplitudes are larger than the coda recordings in these two bands (Fig. S7-8d).

Comodulation-based analysis results: The $\mathrm{ScS}$ amplitude of the $\mathrm{S} 0484 \mathrm{~b}$ event shows a peak in both $\mathrm{SNR}_{\mathrm{w}}$ and $\mathrm{SNR}_{\mathrm{p}}$ of $\sim 1.8$ in the frequency band $0.35-0.5 \mathrm{~Hz}$, while maintaining a value of $\sim 1.4$ for the frequency bands $0.25-0.35 \mathrm{~Hz}$ and $0.5-0.7 \mathrm{~Hz}$ and $0.7-1 \mathrm{~Hz}$.

\section{S0784a:}

Robustness analysis results: We observe that the $\mathrm{ScS}$ is over above the $98 \%$ self-noise at most frequencies from 0.1 to $0.9 \mathrm{~Hz}$. However, only between 0.1 and $0.2 \mathrm{~Hz}$, are amplitudes slightly over the seismic noise in the same local time and coda (Fig. S7-9a and S7-9b). We do not have wind speed or direction data from Sol779 to Sol789, thus we cannot estimate seismic noise due to winds.

Comodulation-based analysis results: The ScS amplitude of the S0784a event shows a peak at $\sim 6$ in the frequency range $0.25-0.35 \mathrm{~Hz}$, and having SNR $>3$ for $0.125-0.5 \mathrm{~Hz}$. Note that this analysis result is not as complete as the other events, as we do not have wind data. 


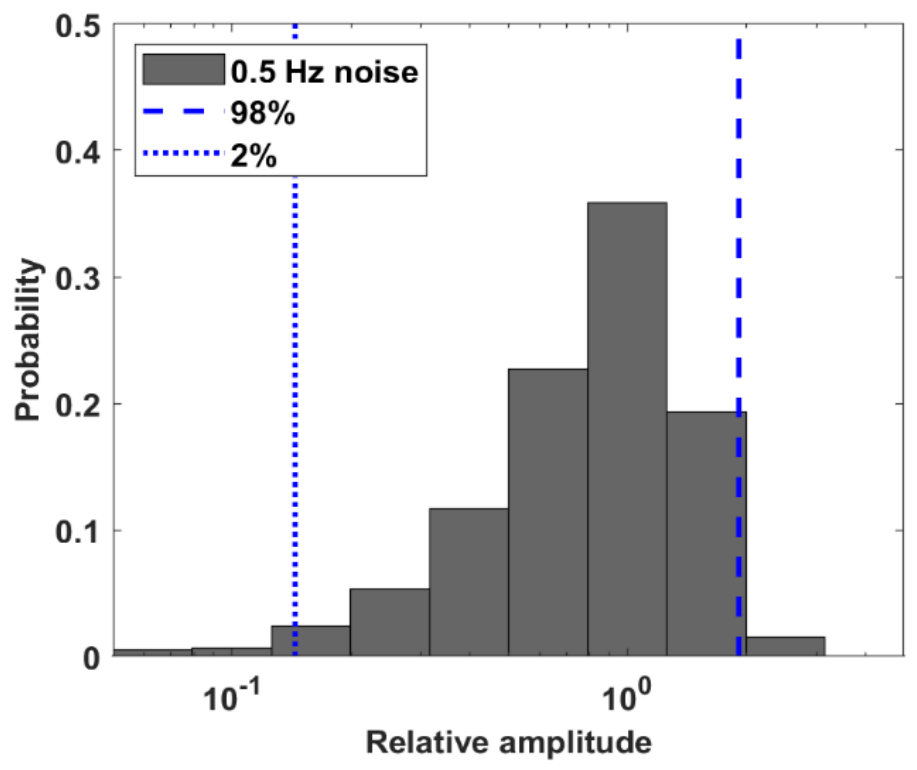

Fig. S7-1. VBB north-component amplitude spectrum distributions for $0.5 \mathrm{~Hz}$ The amplitudes are normalized by the mean value. The dotted and dashed lines indicate the relative (i.e., normalized) amplitudes for $2 \%$ and $98 \%$ distribution locations, respectively. 

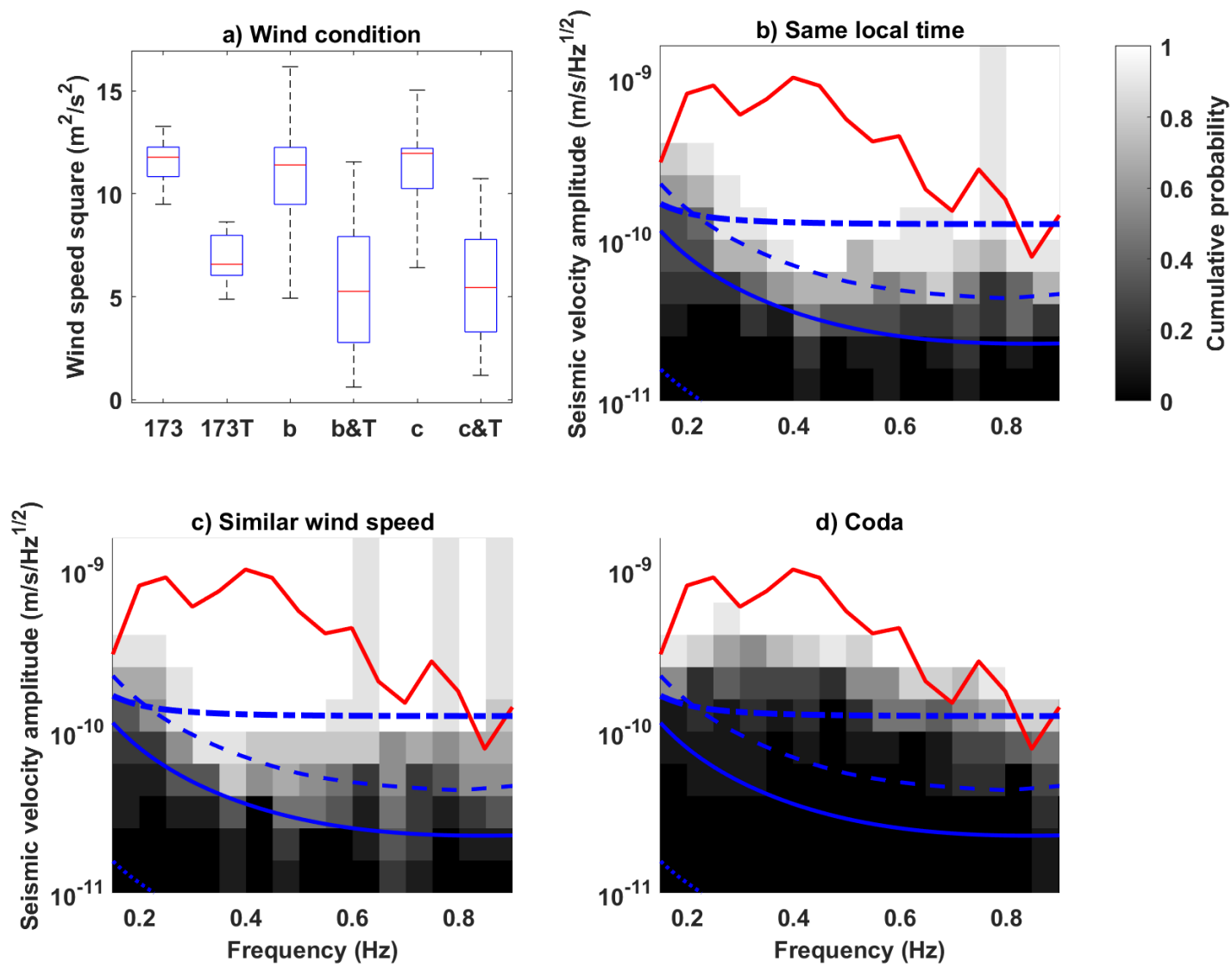

Fig. S7-2. Wind speed and seismic amplitude spectra comparison for S0173a ScS.

a) Six wind-speed-squared distributions shown: for a 1-min long time window centered at the ScS time for Sol 173 (labeled 173), the projection of the wind speed squares to the S0173a transverse (T) direction (labeled 173T), for 1-min long time windows at the same ScS local time from Sol 168 to 178 except 173 (labeled b), the projection of the last distribution to the T direction (labeled b\&T), for 1-min long time windows under a similar wind speed as the $\mathrm{ScS}$ on the 10 Sols (labeled c) and the projection on the T direction (labeled c\&T). The boxes represent $25 \%$ and $75 \%$ of the distributions and the red bars inside are the medians. The black bars are the maxima and minima for each distribution. b) The seismic amplitude spectra for 10s-long time windows at the same time as the wind data b (and b\&T) in a), the same local time of S0173a ScS from Sol 168 to 178 . . c) Similar to b) but for the seismic recordings at the similar-wind-speed time as c (and c\&T) in a). d) We plot the seismic amplitude spectra for 1010 s-long time windows from 100 s to 200 s after the $\mathrm{ScS}$ in cumulative probability. In (b-d), we plot the spectra in cumulative probability, indicated by the gray color bar, and the ScS spectrum as the red curve. The solid blue curve is the VBB instrument noise. The dashed and dotted blue curves indicate the $98 \%$ and $2 \%$ of VBB self-noise, respectively. The thick dash-dotted blue line is our estimate for the ground velocity due to the wind speed squared at S0173a ScS time. 
a

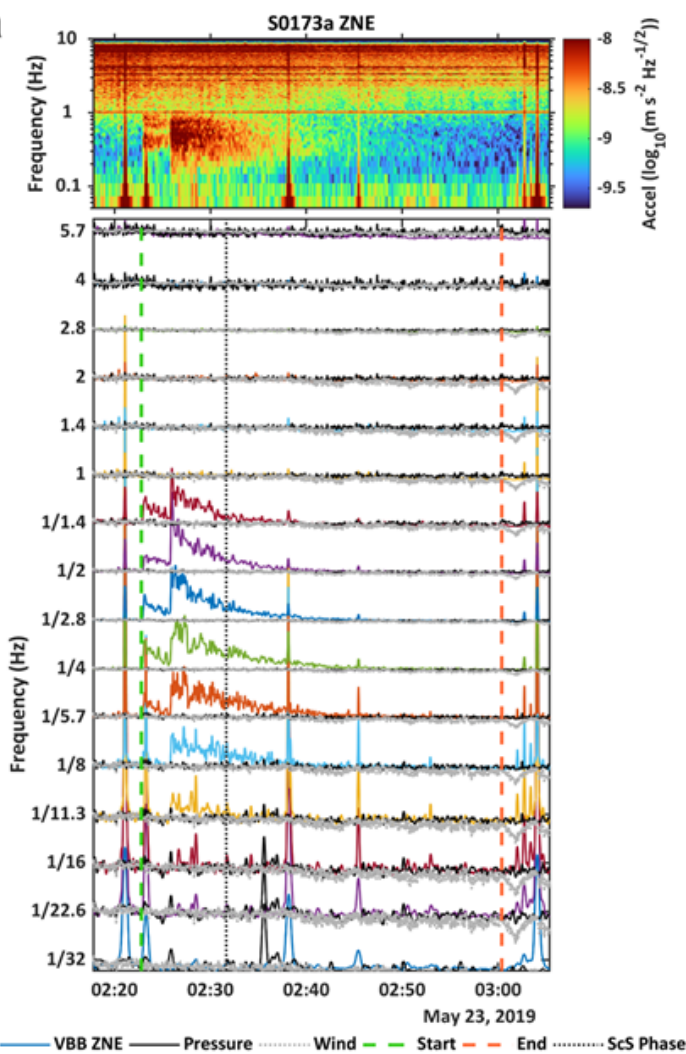

\section{b}
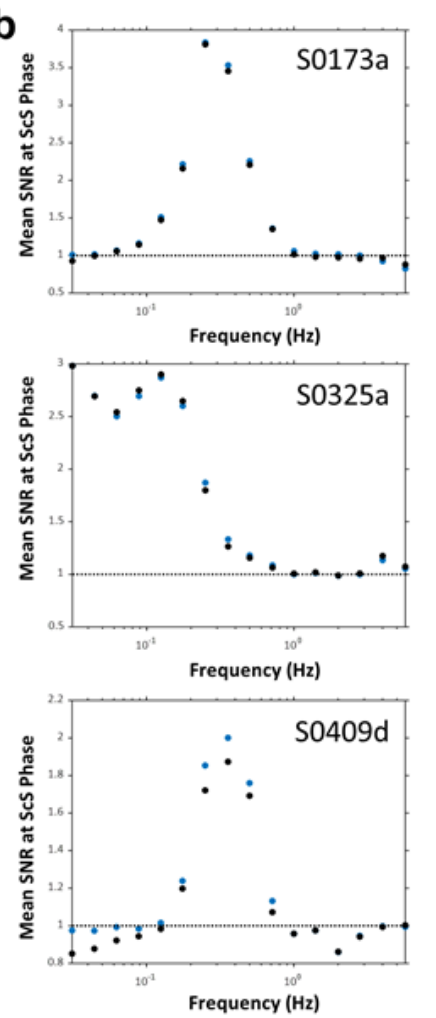

- Wind • Pressure
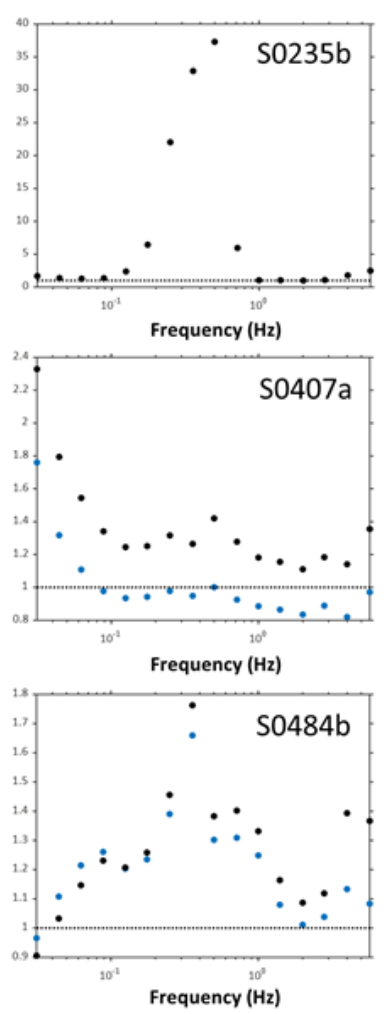

Frequency $(\mathrm{Hz})$

\section{Fig. S7-3. Comodulation analysis for S0173a}

(a, top) Spectrogram of the combined total power of all three Z, N and E components for the S0173a event (a, bottom) Set of envelopes extracted in half-octave frequency bands of the combined ZNE seismic acceleration (colored lines) and pressure (black) moment-matched to the measured wind speed (dotted grey line) using the mean and variance as estimated prior to the start of the S0173a event. The start and end of the event is denoted by the green and orange dashed lines, respectively. The start of the ScS phase is denoted by the dotted black line. (b) Mean SNR values obtained at the candidate ScS phases across different frequency bands for the Marsquake events S0173a, S0235b, S0325a, S0407a, S0409d and S0484b respectively. 

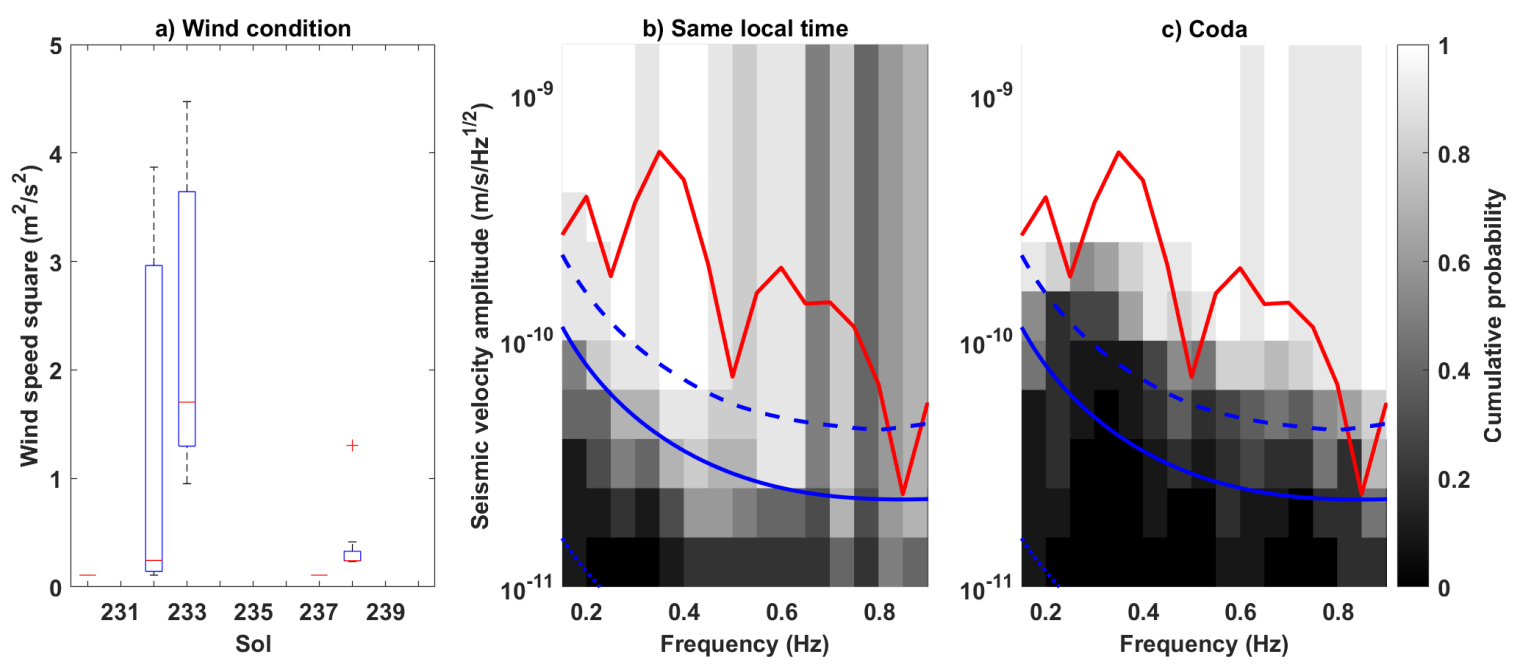

Fig. S7-4. Wind speed and seismic amplitude spectra comparison for S0235b ScS.

All data processing parameters are the same as for Fig. S7-2 apart from those corresponding to the wind. We plot available wind speed squares from Sol 230 to 240 with data gaps on most of the Sols. We do not have the wind speed data at the $\mathrm{ScS}$ local time. The symbols in a) are the same as in Fig. S7-2a. 

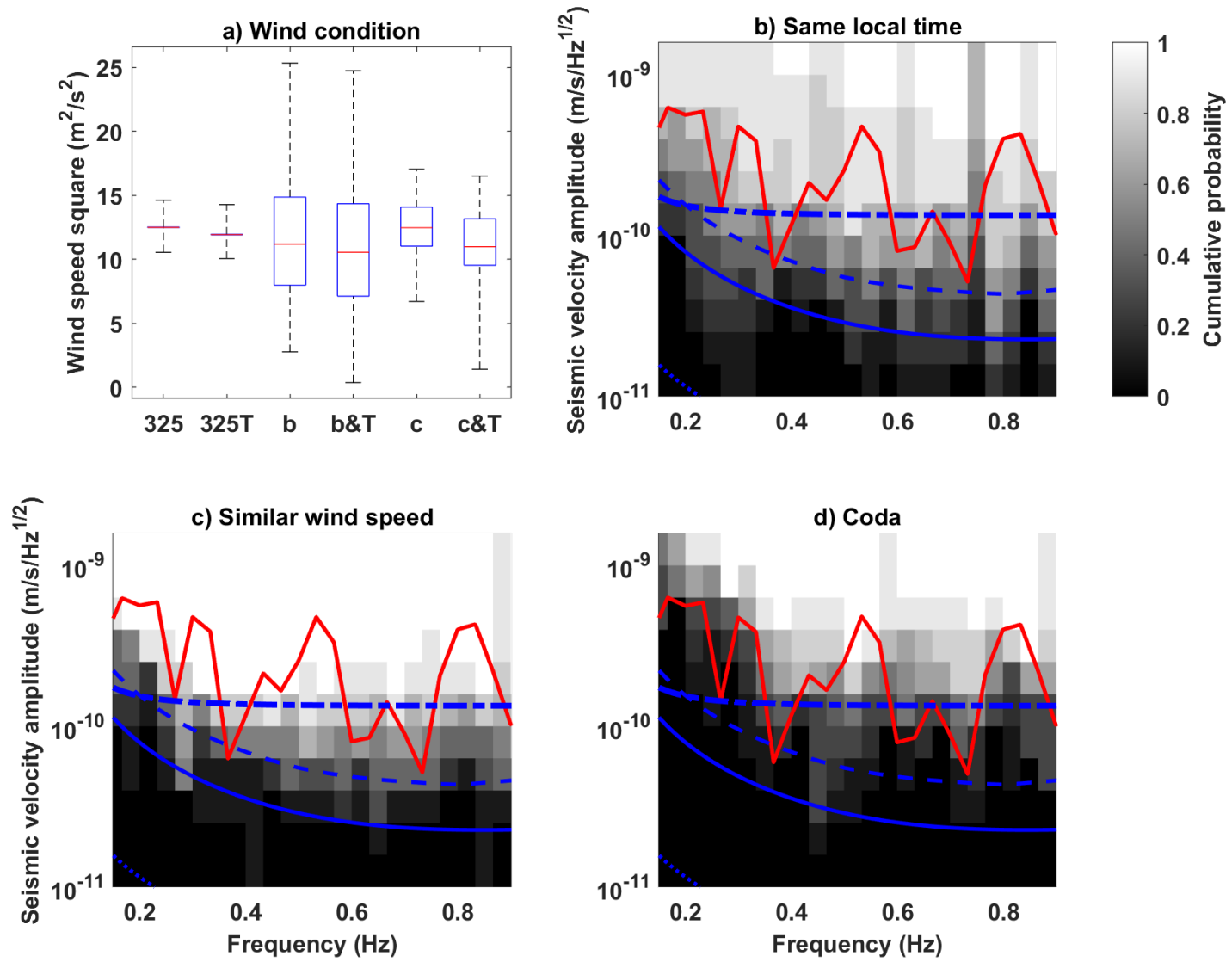

Fig. S7-5. Wind speed and seismic amplitude spectra comparison for S0325a ScS.

Data and results are displayed as in Fig. S7-2. 

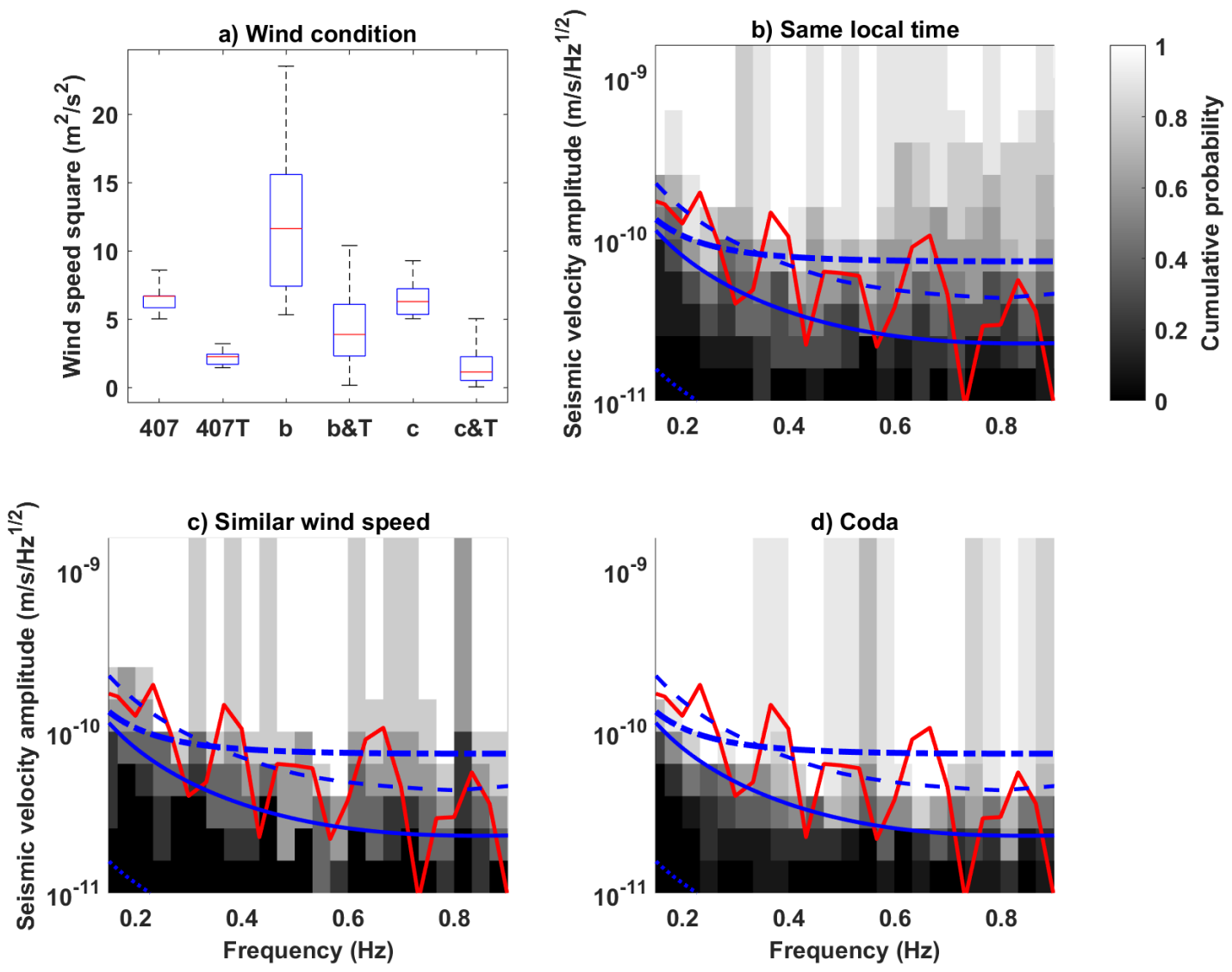

Fig. S7-6. Wind speed and seismic amplitude spectra comparison for S0407a ScS. All data processing parameters match those in Fig. S7-2 except that the time windows are increased from 10s to 20s in length. In a) the wind speed data are from Sol 402 to 413. S0407a backazimuth is $90 \mathrm{deg}$ and $\mathrm{ScS}$ starts at UTC 2020-01-19T10:6:10. 

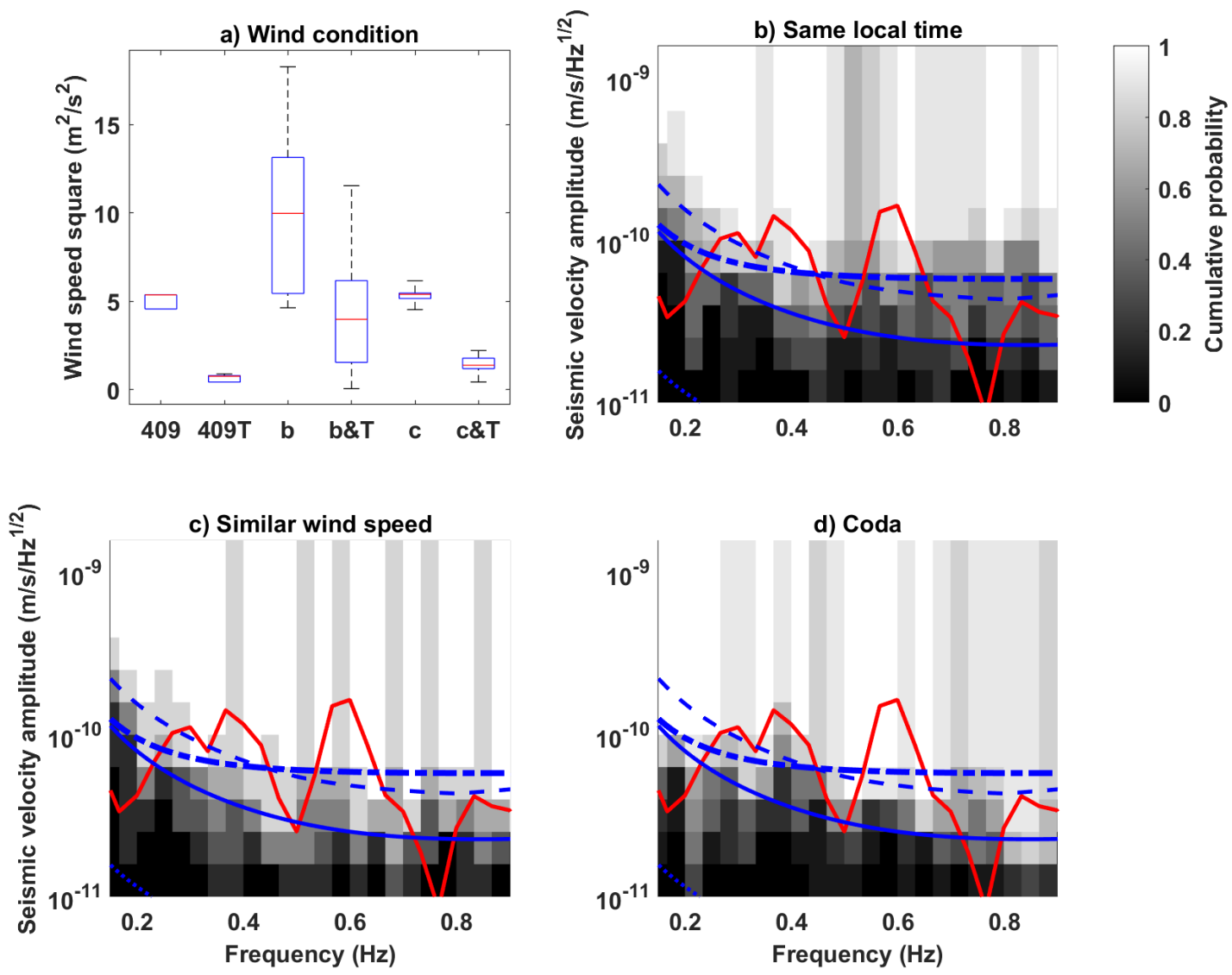

Fig. S7-7. Wind speed and seismic amplitude spectra comparison for S0409d ScS. All data processing parameters match those in Fig. S7-2. In a) the wind speed data are from Sol 404 to 414. S0409d backazimuth is $90 \mathrm{deg}$ and ScS starts at UTC 2020-1-21T11:39:50. 

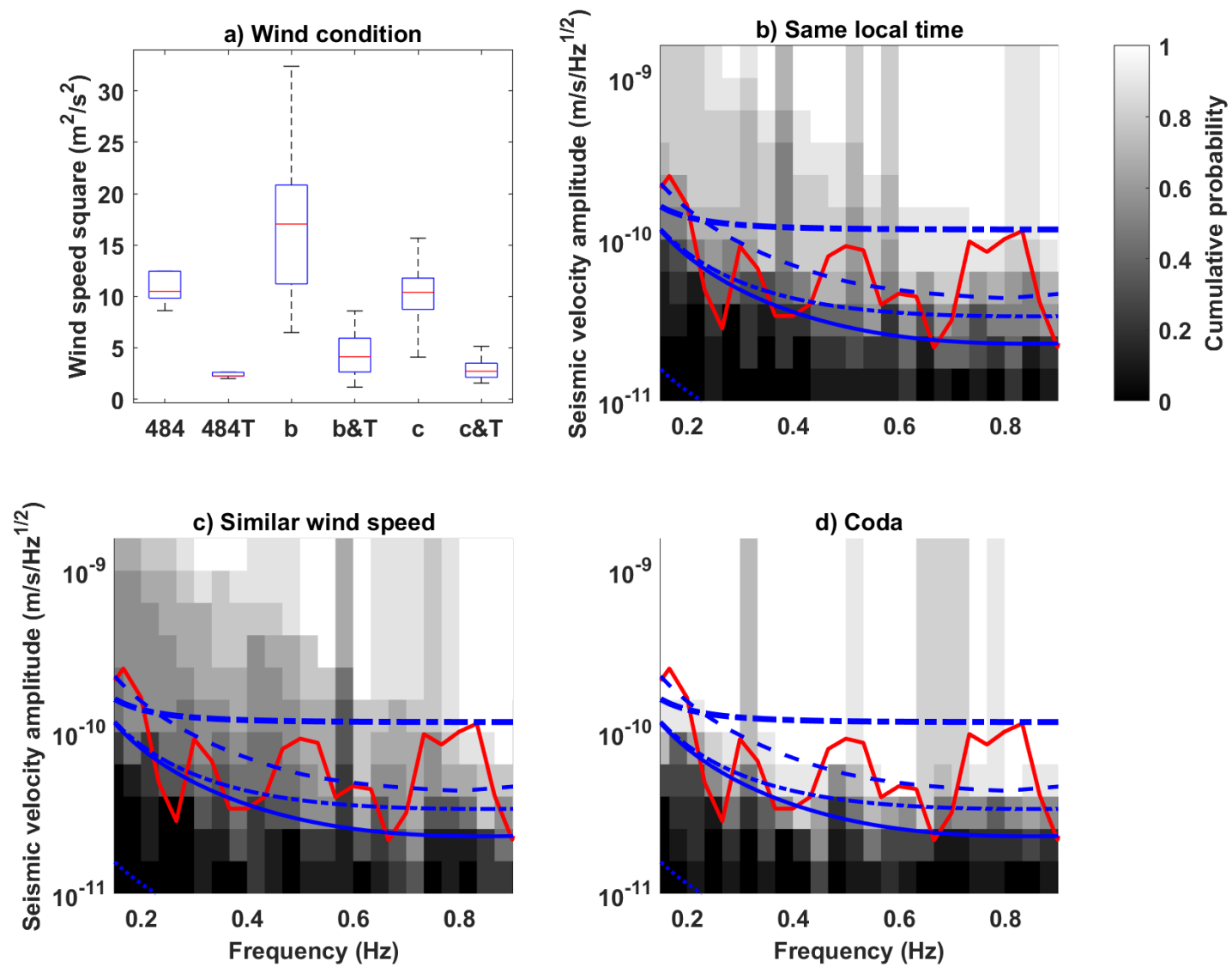

Fig. S7-8. Wind speed and seismic amplitude spectra comparison for S0484b ScS. All data processing parameters match those in Fig. S7-2. In a) the wind speed data are from Sol 479 to 484 . In addition to the ground velocity estimates based on the wind speed squares at the $\mathrm{ScS}$ time (thick dash-dotted blue line), we also estimate the ground velocities based on the projection of the wind speed squares on the T-component direction (thin dash-dotted blue line). The S0484b backazimuth is $90 \mathrm{deg}$ and ScS starts at UTC 2020-04-07T09:00:50. 

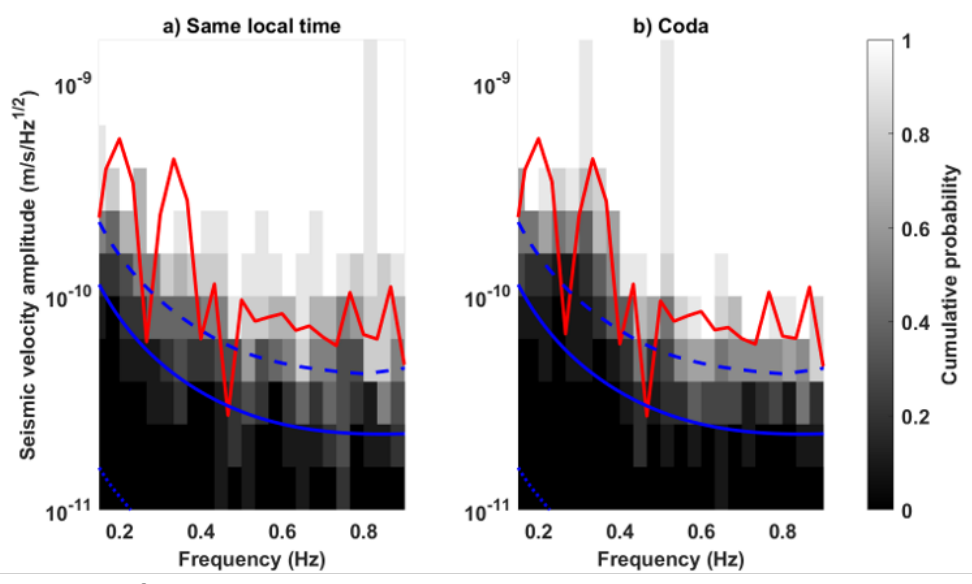

c)

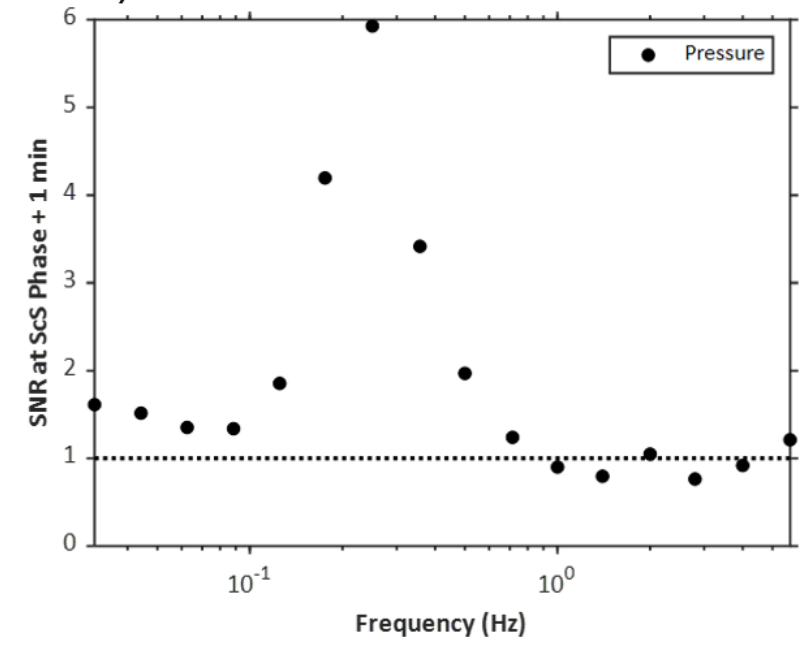

Fig. S7-9. The seismic amplitude spectra comparison for and the mean SNR values obtained at S0784a ScS.

All data processing parameters are those of the processing made for Fig. S7-2. We do not have the wind speed data from Sol779 to Sol789. The symbol settings in a), b) and c) are the same as in Fig. S7-2b,2d and Fig. S7-3b, respectively. The S0784a backazimuth is 90 deg and ScS starts at 2021-02-09T12:24:25 


\section{Model parameterization}

This section describes the three different parameterizations used to create models of Mars' internal properties - specifically a "seismic" parameterization, a "geophysical" parameterization and a "geodynamic" parameterization. The inversion method is discussed in section eight, and the models produced using the three different parameters are displayed in the main text.

\section{$\underline{\text { 8.1 Seismic model parameterization }}$}

We inverted differential travel times using a "standard" seismic parameterization, i.e., layered models with variable P- and S- wave velocities and variable depth nodes. In contrast to the other methodologies, this approach provides increased flexibility in determining the velocity models, since it allows inversion for both P- and S-wave velocities independently of any thermodynamic and petrologic property, or thermo-chemical evolution of the planet.

To retrieve the general features of the velocity structure, we consider a simplified model that is divided into three crustal layers and six mantle nodes. In the crust, each layer is parameterized in terms of variable thickness and variable P-to-S-wave velocity scaling, while in the mantle we consider variable depth nodes and independent $\mathrm{P}$ - and $\mathrm{S}$-wave velocities.

Crustal velocities are assumed to increase as a function of depth, while mantle velocities are free to sample the a priori velocity range. The a priori model parameter information, which consists of constraints on absolute P- and S-wave velocities and absolute depth nodes, is summarized in Table S8-1. As a part of the inversion, we also determine epicentral distance for each event.

To infer velocity structure and epicentral distance for all events, we invert the differential observed body wave phase picks based on time-domain envelope picks (32) and the core phases that are tabulated in Table 1. For this purpose, we use the Bayesian framework explained in (32). To sample the posterior distribution, we employ the Metropolis Hastings algorithm, assuming that error can be modeled using an exponential probability density. Sampling is performed using ten independent chains with a total length of $10^{4}$ iterations, characterized by identical initial models but different randomly chosen initial perturbation. Finally, an interval including the $95 \%$ credible intervals of the sampled inverted radial P- and S-wave velocity profiles for the seismic parameterization is shown in Fig. 2 in the main text. This range is based on the 30,000 bestfitting candidates.

Due to the use of ScS picks, the current set of differential travel time data allow us to constrain the S-wave velocity profile down to the core-mantle boundary, while for P-wave velocity we can only constrain down to $\sim 800 \mathrm{~km}$ depth. In analogy with the approach described in (32) we simultaneously inverted for the epicentral distance of all events considered here. Since the results are similar, we do not show these here. 
Submitted Manuscript: Confidential

Template revised February 2021

\begin{tabular}{|l|l|c|l|l|}
\hline Description & Quantity & Parameter & Value/Range & Distribution \\
\hline $\begin{array}{l}\text { Crustal } \\
\text { properties }\end{array}$ & 3 & $V_{S}$ & $2-4.2 \mathrm{~km} / \mathrm{s}$ & uniform \\
\cline { 2 - 6 } & - & $V_{P}$ & $\alpha \cdot V_{S}$ & \\
\hline Vp/Vs scaling & 1 & $\alpha$ & $1.65-1.85$ & $V_{S / P}^{1} \leq V_{S / P}^{2} \leq V_{S / P}^{3}$ \\
\hline Mantle properties & 6 & $V_{S}$ & $3-6.5 \mathrm{~km} / \mathrm{s}$ & uniform \\
\cline { 2 - 6 } & 6 & $V_{P}$ & $6-12 \mathrm{~km} / \mathrm{s}$ & uniform \\
\hline Crustal thickness & 3 & $\Delta Z_{i}^{c}$ & $5-50 \mathrm{~km}$ & uniform \\
\hline Mantle nodes & 6 & & $20 \mathrm{~km} \leq \sum_{i} \Delta Z_{i} \leq 60 \mathrm{~km}$ & \\
\hline $\begin{array}{l}\text { Epicentral } \\
\text { distances }\end{array}$ & 9 & $Z_{i}^{m}$ & Core radius: $Z_{6}^{m}<2000 \mathrm{~km}$ & uniform \\
\hline Source depth & 9 & \multicolumn{2}{|c|}{$\begin{array}{l}\text { Shown in Table } 1 \text { of the main } \\
\text { paper }\end{array}$} & fixed \\
\hline
\end{tabular}

Table S8-1. Seismic model parameterization and prior model parameter information. 


\subsection{Geophysical model parameterization}

The seismic velocity models of Mars discussed in the main manuscript that are based on the geophysical parameterization follow the approach of (4). Here we adhere to the description in (32). Radial profiles of mantle seismic P- and S-wave velocity and density are computed using petrologic phase-equilibrium computations in the NCFMAS model chemical system that comprises the oxides $\mathrm{CaO}-\mathrm{FeO}-\mathrm{MgO}-\mathrm{Al}_{2} \mathrm{O}_{3}-\mathrm{SiO}_{2}-\mathrm{Na}_{2} \mathrm{O}$. From a practical point of view, we employ Gibbs free-energy minimization to compute stable mantle mineralogy and physical properties as a function of temperature, composition, and pressure based on the thermodynamic formulation and parameters of $(113,114)$. The virtue of this parameterization is that it relies on a unified description of phase equilibria, seismic properties, and thermo-chemical parameters. Major sources of uncertainty in the thermodynamic calculations are the absence of experimental constraints on the parameters relevant for the thermodynamic formalism and parameterization of (113-115). (115) estimated the accuracy of the elastic moduli and density to be $\sim 0.5 \%$ and $\sim 1-2$ $\%$, respectively.

Martian geotherms are parameterized by a conductive crust and lithosphere underneath which the mantle is assumed to be adiabatic (Fig. S8-2). The conductive part is defined by two linear thermal gradients (blue lines in Fig. S8-2): the first is between the surface and the bottom of the crust-mantle interface (of variable thickness Z' and temperatures T') and the second between the crust-mantle interface and the base of the lithosphere (of variable thickness $Z_{\mathrm{lit}}$ and temperature $\mathrm{T}_{\mathrm{lit}}$ ), respectively. Because of enrichment in heat-producing elements, a moderate curvature may develop in the crustal thermal gradient. However, the exact nature of the crustal geotherm is unimportant for the inversion since we resorted to a seismic parameterization of the crust as described above.

For the crust, we rely on a "standard" seismic parameterization (P- and S-wave velocity) and consider a 3-layer crust with variable depth nodes and variable P-to-S-wave and density-to$\mathrm{S}$-wave velocity scaling. This assumption of crustal seismic structure decoupled from temperature originates from the fact that the crustal seismic structure of Mars may be dominated by various distinct processes that do not directly or primarily relate to temperature such as alteration, porosity, compositional and structural heterogeneity, and fracturing/damage (8).

The thermal structure of the mantle is assumed to be adiabatic and the adiabats (isentropes) are computed self-consistently from the entropy of the lithology at the pressure and temperature equivalent of the bottom of the lithosphere (112). The mantle pressure profile is obtained by integrating the vertical load from the surface pressure boundary condition.

For the parameterization of the Martian core in the Fe-FeS system, we follow the approach of (16) and assume that Mars' core is well-mixed and convecting. To compute depth-dependent thermoelastic properties for the core, we use equations-of-state for liquid iron and liquid ironsulfur alloys as described in detail in (16). Core parameters include radius, composition (S content), and the input parameters required to compute physical properties of the core are those determined by integrating the load from the surface to the CMB and the entropy of the lithology at $\mathrm{T}_{\text {lit }}$, which determines the temperature at the CMB.

The prior model parameter information is summarized in Table S8-2 below. The chosen prior ranges represent the information acquired from data and results from experimental and numerical studies as discussed in the foregoing sections. In analogy with the approach described in (32) we simultaneously inverted for the epicentral distance of all events considered here. Since the results are similar, we do not show these here. 


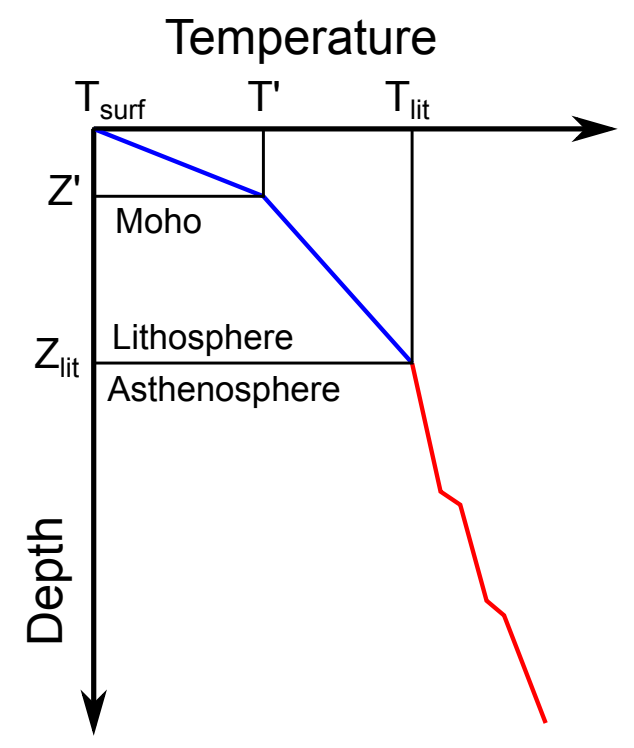

Fig. S8-2. Parameterization of crust and mantle geotherm.

$\mathrm{T}_{\text {surf, }} \mathrm{T}$ ', and $\mathrm{T}_{\text {lit }}$ signify temperatures at the surface and the bottom of the crust and lithosphere, respectively. $Z^{\prime}$ and $Z_{\text {lit }}$ refer to the thickness of crust and lithosphere, respectively. Blue and red lines indicate conductive and adiabatic parts of the geotherm, respectively. 
Submitted Manuscript: Confidential

Template revised February 2021

\begin{tabular}{|c|c|c|c|c|}
\hline Description & Quantity & Model parameter & Value/Range & Distribution \\
\hline Crustal thickness & 1 & $Z^{\prime}$ & $20-60 \mathrm{~km}$ & log-uniform \\
\hline Crustal properties & 3 & $\mathrm{Vs}_{\mathrm{s}}$ & $2-4.2 \mathrm{~km} / \mathrm{s}$ & log-uniform \\
\hline $\begin{array}{l}\text { First crustal layer } \\
\text { thickness }\end{array}$ & 1 & $\mathrm{Z}_{1}$ & $5-15 \mathrm{~km}$ & log-uniform \\
\hline $\begin{array}{l}\text { Crust-mantle } \\
\text { interface } \\
\text { temperature }\end{array}$ & 1 & $\mathrm{~T}^{\prime}$ & $473-1273 \mathrm{~K}$ & log-uniform \\
\hline $\begin{array}{l}\text { Crustal Vp/Vs } \\
\text { scaling }\end{array}$ & 1 & $\begin{array}{l}\alpha \\
\left(\mathrm{V}_{\mathrm{P}}=\alpha \mathrm{V}_{\mathrm{S}}\right)\end{array}$ & $1.65-1.85$ & log-uniform \\
\hline $\begin{array}{l}\text { Crustal } \rho / V s \\
\text { scaling }\end{array}$ & 1 & $\begin{array}{l}\beta \\
\left(\rho=\beta V_{s}\right)\end{array}$ & $0.75-1$ & log-uniform \\
\hline $\begin{array}{l}\text { Lithospheric } \\
\text { temperature }\end{array}$ & 1 & $\mathrm{~T}_{\text {lit }}$ & $1273-1873 \mathrm{~K}$ & log-uniform \\
\hline $\begin{array}{l}\text { Lithospheric } \\
\text { thickness }\end{array}$ & 1 & $Z_{\text {lit }}$ & $100-600 \mathrm{~km}$ & log-uniform \\
\hline $\begin{array}{l}\text { Mantle } \\
\text { composition }\end{array}$ & - & $\mathrm{X}_{\mathrm{m}}$ & $10,12,56-59$ & fixed \\
\hline Core radius & 1 & $\mathrm{R}_{\text {core }}$ & $1000-2500 \mathrm{~km}$ & log-uniform \\
\hline Core $\mathrm{S}$ content & 1 & $\mathrm{Xs}_{\mathrm{s}}$ & $0-0.5$ & log-uniform \\
\hline Epicentral distance & 9 & $\Delta$ & $0^{\circ}-180^{\circ}$ & log-uniform \\
\hline Source depth & 9 & $\mathrm{~h}$ & $\begin{array}{l}\text { Table } 1 \text { (main } \\
\text { paper) }\end{array}$ & fixed \\
\hline
\end{tabular}

Table S8-2. Geophysical model parameterization and prior model parameter information. 


\subsection{Geodynamic model parameterization}

The geodynamically-constrained models discussed in the main manuscript are based on the approach described in $(41,116)$. This approach relies on a parameterization in terms of quantities that influence the thermo-chemical evolution of the planet (mantle rheology, initial thermal state, and composition), which accounts for 4.5 Gyr of planetary evolution. The resulting present-day thermo-chemical state of the planet is used to compute the density, and P-and S- waves seismic velocity profiles of Mars assuming a compositional, a mineralogical, and a thermodynamic model.

The parameterized model considers a planet divided into three concentric and spherically symmetric envelopes:

1. a convecting liquid iron-rich core;

2. an adiabatic convecting silicate mantle (with top and bottom thermal boundary layers);

3. a time-evolving stagnant lithospheric lid, which includes a crust enriched in heatproducing elements with respect to the underlying mantle.

The thermo-chemical evolution is computed following the approach in (42) and references therein. This evolution is computed by accounting for the conductive and convective heat transfer through and between the aforementioned planetary envelopes. This approach reproduces the evolution of a Mars-sized stagnant lid planet in spherical geometry well, both in the cases of transient and steady-state evolution. It includes the effects of complexities such as temperature and pressure-dependent mantle viscosities, and the presence of a crust whose thickness progressively evolves via melt extraction at shallow pressure. The ratio of heat-producing elements content in the crust relative to that of the primitive mantle is set to 10 , which is in line with geochemical compositional models and estimates of surface abundances inferred from Gamma Ray Spectrometer data (117). The mantle rheology (i.e., its reference viscosity, $\eta_{0}$, and its effective activation energy, $E^{*}$ ) and the initial thermal state (i.e., the core mantle boundary $T_{c 0}$, and the uppermost mantle temperature, $T_{m 0}$ ) are not well constrained, and exert an important influence on the thermal evolution. Therefore, we invert for these quantities. The mantle effective activation volume $V^{*}$ that expresses the sensitivity of viscosity with pressure is constrained to be lower than $4 \mathrm{~cm}^{3} / \mathrm{mol}$ (79) and exerts only a weak influence on the thermochemical evolution below this value, we therefore set $V^{*}$ to $3 \mathrm{~cm}^{3} / \mathrm{mol}$. The crustal seismic structure is decoupled from temperature, and is directly inverted for. This assumption originates from the understanding that the crustal seismic structure of Mars may be dominated by various distinct processes that do not directly or primarily relate to temperature such as alteration, porosity, compositional and structural heterogeneity, and fracturing/damage (8). In contrast, in the lithosphere below the crust and in the mantle $\mathrm{P}$ - and $\mathrm{S}$-wave velocities and density are computed from the obtained thermo-chemical state at the present-day. Such computations of density and seismic velocities from thermal profiles are performed using a thermodynamic model via the Perple_X Gibbs free energy minimization software (118), using the formulation and database of $(113,114)$. In the mantle, the composition of (45) is used, because among other plausible Martian mantle compositions, (33) have demonstrated that this composition is compatible with the moment of inertia and crust density and thickness estimations deduced from receiver function estimations and gravity and topography inversions. The core density is adjusted in order to match the mean mass of Mars, $M=6.417 \times 10^{23} \pm 2.981 \times 10^{19} \mathrm{~kg}(51)$. The prior model parameter information described above is summarized in Table S8-3. 


\begin{tabular}{|l|l|l|}
\hline Description & Value/Range & Distribution \\
\hline Initial uppermost mantle temperature, $T_{m} 0$ & $1700-2000 \mathrm{~K}$ & Gaussian \\
\hline Initial temperature at the CMB, $T_{c} 0$ & $T_{c 0}-T_{m 0}=300 \mathrm{~K}$ & Depends on $T_{m} 0$ \\
\hline Effective mantle activation energy, $E^{*}$ & $60-500 \mathrm{~kJ} / \mathrm{mol}$ & Gaussian \\
\hline Reference mantle viscosity, $\eta_{0}$ & $10^{20}-10^{22.5} \mathrm{~Pa} \mathrm{~s}$ & Gaussian \\
\hline Core radius, $R_{c}$ & $1500-2000 \mathrm{~km}$ & Gaussian \\
\hline Mantle $V_{S}$ factor & $0.95-1.05$ & Gaussian \\
\hline Mantle $V_{p}$ factor & $0.95-1.05$ & Gaussian \\
\hline$V_{S}$ in the upper crust (layer 1$)$ & $1.0-3.0 \mathrm{~km} / \mathrm{s}$ & Gaussian \\
\hline$V_{S}$ in the mid-crust (layer 2) & $2.0-3.5 \mathrm{~km} / \mathrm{s}$ & Gaussian \\
\hline$V_{S}$ in the lower crust (layer 3) & $3.9-4.4 \mathrm{~km} / \mathrm{s}$ & Gaussian \\
\hline$V_{P} / V_{S}$ in the entire crust & $1.7-1.9$ & Gaussian \\
\hline Epicentral distance & $0-180^{\circ}$ & Gaussian \\
\hline Source depth & Table $1(\mathrm{main}$ paper) & Fixed \\
\hline
\end{tabular}

Table S8-3. Geodynamical model parameterization and prior model parameter information. 


\section{Inversion}

In the following, we illustrate the inversion on the basis of the geodynamical method, since the same stochastic sampling method is employed across all three parameterizations described in the previous section. We then test the results of the inversion using the geodynamical method using different combinations of input data.

\subsection{Practical implementation}

Due to the ill-posed nature of the problem, we use a Bayesian approach based on a Markov chain Monte Carlo (McMC) method (e.g., (48)) to solve the inverse problem. For this, we follow the procedure described by $(41)$.

The models are sampled according to the prior, which represents our current state of knowledge. The parameters are sampled within the bounds listed in Table S8-3. In total, 10 parameters are used to build the seismic model. Three layers are considered in the crust. The total crustal thickness is directly estimated by the thermal evolution model. The thickness of each crustal layer is estimated by considering that the upper, middle, and lower layers are $10 \%, 40 \%$, and $50 \%$ of the total crustal thickness, respectively. Along the inversion process, the average crustal density is adjusted within the interval $2500-3100 \mathrm{~kg} / \mathrm{m}^{3}$ in order to match the Moment of Inertia (MoI) estimate for Mars: $I /\left(M R^{2}\right)=0.36340 \pm 0.00006$ ( 11 ). If no crustal density within the aforementioned range yields a MoI compatible with the observation, the model is rejected.

In the McMC algorithm new models are proposed by performing a random walk in the model space. For this, we employ the Metropolis-Hastings sampler with Gaussian proposal distributions. All the parameters are simultaneously randomly sampled. Figure S9-1 shows the $a$ priori distribution of $V_{S}$ and $V_{P}$ as a function of depth, given the prescribed conditions detailed in Table S8-3. Both the a priori assumptions and the sampling of the models lead to non-uniform distributions at a given depth. However, a large parameter space is considered to ensure that no acceptable solutions are missed a priori.

Following the work of $(41,116)$, we performed a MPI-parallel two-step inversion scheme to speed up convergence. During the first step, a broad exploration of the model space is performed by randomly perturbing the parameters listed in Table S8-3 using wide Gaussian proposal distributions. To allow the algorithm to sample a sufficient number of extrema in the model space, we ran 96 independent Markov chains in parallel over 300 iterations starting in a different location of the model space and with a different random seed. The best-fitting model is then determined for each chain and the models are sorted in ascending order based on their misfit values. To discard the chains that may have failed to converge, the first 48 configurations with the smallest misfits of the 96 best-fitting models are selected to be the starting models of the second step (or stationary stage), which will be used to compute the final statistics. To further ensure that the chains have reached an acceptable solution before moving on to the second step, we verified that the synthetic seismic data from the 48 configurations with the smallest misfits fit the observations within uncertainties. We then ran 48 independent chains in parallel for 4000 iterations during the second step, sampling the parameter space with narrower Gaussian proposal distributions. Given that the McMC method provides a series of dependent samples, the statistics on core radius and degree-two Love number, $k_{2}$, were computed using a subset of 10000 models in order to reduce the correlation between the sampled models. To simplify the comparison with the other inversion approaches, we excluded the contribution of anelastic effects in calculating 
$k_{2}$, which depends on the shear attenuation profile in the mantle. Nevertheless, anelastic contributions to $k_{2}$ are likely to be small if the mantle remains in a solid state $(79,119)$.

The body waves arrival times for a given model are then calculated using the TauP software (120). Note that only the first arrival of each seismic phase is considered. Since the origin time of the seismic event is unknown (because the InSight seismic network consists of a single seismic station), in the misfit function we use differential arrival times relative to the P-wave phase arrival for the PP, and PPP phases, and relative to the S-wave phase arrival for the SS, SSS, and $\mathrm{ScS}$ phases.

\subsection{Results}

To investigate to what extent the core radius could be constrained using the available data, we performed three different inversions by considering (1) the current seismic data, (2) geodetic data, and (3) both seismic and geodetic data. The geodetic data consists of the $k_{2}$ that is corrected for atmospheric effects, i.e., $k_{2}=0.174 \pm 0.008$ as described in (11).

The retrieved $V_{S}$ and $V_{P}$ profiles for the three cases are shown in Figures S9-2 and S9-3. For a better analysis of the results, two different representations are used to investigate the $V_{S}$ and $V_{P}$ distributions. Figure S9-2 displays the a posteriori probability density functions (pdfs). The pdfs provide an overview of the most frequently sampled models, and show the additional gain in information obtained through inversion, compared to the a priori distribution (Fig. S9-1). Figure S9-3 shows 200 models randomly selected in the ensemble models. This representation highlights the diversity of the models sampled. Figures S9-2 and S9-3 show that the distributions of the seismic velocity profiles are spread in the parameter space considering geodetic data only, compared to the distributions using seismic data only. When using both seismic and geodetic data, the pdfs are narrower and exhibit larger values (panels a3 and b3 in Fig. S9-2). The $V_{S}$ profiles decrease between the base of the crust and approximately $400 \mathrm{~km}$ depth. This low velocity zone is due to the large thermal gradient across the lithosphere, which dominates over the increase of velocity due to the increase of pressure with depth. For all cases, $V_{P}$ is less constrained than $V_{S}$, because of a smaller number of identified PP and PPP phases compared to the SS and SSS phases.

Note that all these models are able to fit the differential travel time data within error bounds, as shown in Fig. S9-4. The Fig. S9-4 (a1-a2) and (b1-b2) reveal a trade-off between $t_{P p}-t_{p}, t_{P p P}-t_{P}$, $t_{S S}-t_{S}, t_{S S S}-t_{S}$, and $t_{S}-t_{P}$. This implies that when seismic velocities in the mantle decrease, the arrival times of $\mathrm{P}$ and S-waves, and their multiples increase, and vice versa. Conversely, Fig. S9$4(\mathrm{c} 1)$ and (c2) indicate that $\mathrm{t}_{\mathrm{ScS}}-\mathrm{t}_{\mathrm{S}}$ decreases when $\mathrm{t}_{\mathrm{S}}-\mathrm{t}_{\mathrm{P}}$ increases. Indeed, lower seismic velocities in the mantle imply a larger core to fit the moment of inertia, and thus the ScS phases arrive earlier. The retrieved epicentral distances are not shown here, but are in good agreement with the results from (32).

The resulting distributions of the core radius, $k_{2}$ values, and core density, are displayed in Fig. S9-5. The mean values and standard deviation of the a posteriori distribution are displayed in red. The retrieved core radii using seismic data alone and geodetic data alone are $1836 \pm 61 \mathrm{~km}$ and $1815 \pm 70 \mathrm{~km}$, respectively (Fig. S9-5 (a1) and (a2)). The combination of seismic and geodetic data reduces the standard deviation, yielding a mean core radius with a 1-sigma range of $1827 \pm 40 \mathrm{~km}$ (Fig. S9-5 (a3)). The addition of $k_{2}$ into the misfit function results in the exclusion of models with core radius values located at the edges of the distributions obtained using either seismic data only or geodetic data only. Core density values are in good agreement among the three inversion results, with a value of $6.08 \pm 0.13 \mathrm{~g} / \mathrm{cm}^{3}$ for the case where both seismic and geodetic data are used. 


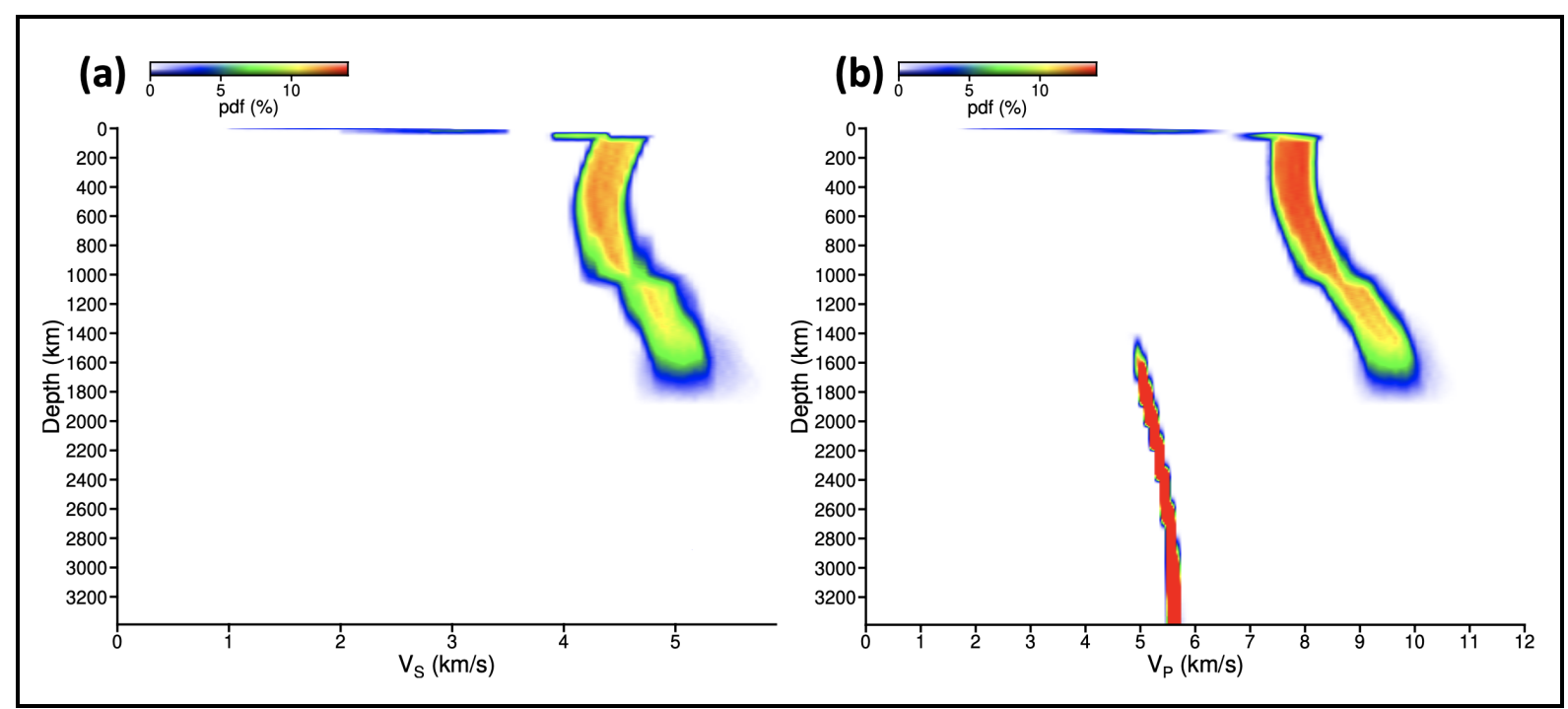

Fig. S9-1. A priori probability density functions (pdfs) of the 1-D (a) $V_{S}$ and (b) $V_{P}$ profiles.

These are all the sampled models compatible with the a priori information detailed in Table S83. Red and blue colors refer to low and high probabilities, respectively. The pdfs are computed by counting the number of sampled profiles for each case. The spacing is $1 \mathrm{~km}$ for depth, and $0.05 \mathrm{~km} / \mathrm{s}$ and 0.1 for $V_{S}$ and $V_{P}$, respectively. At each given depth, the sum of the pdf over all the velocity intervals equals 100 percent. 


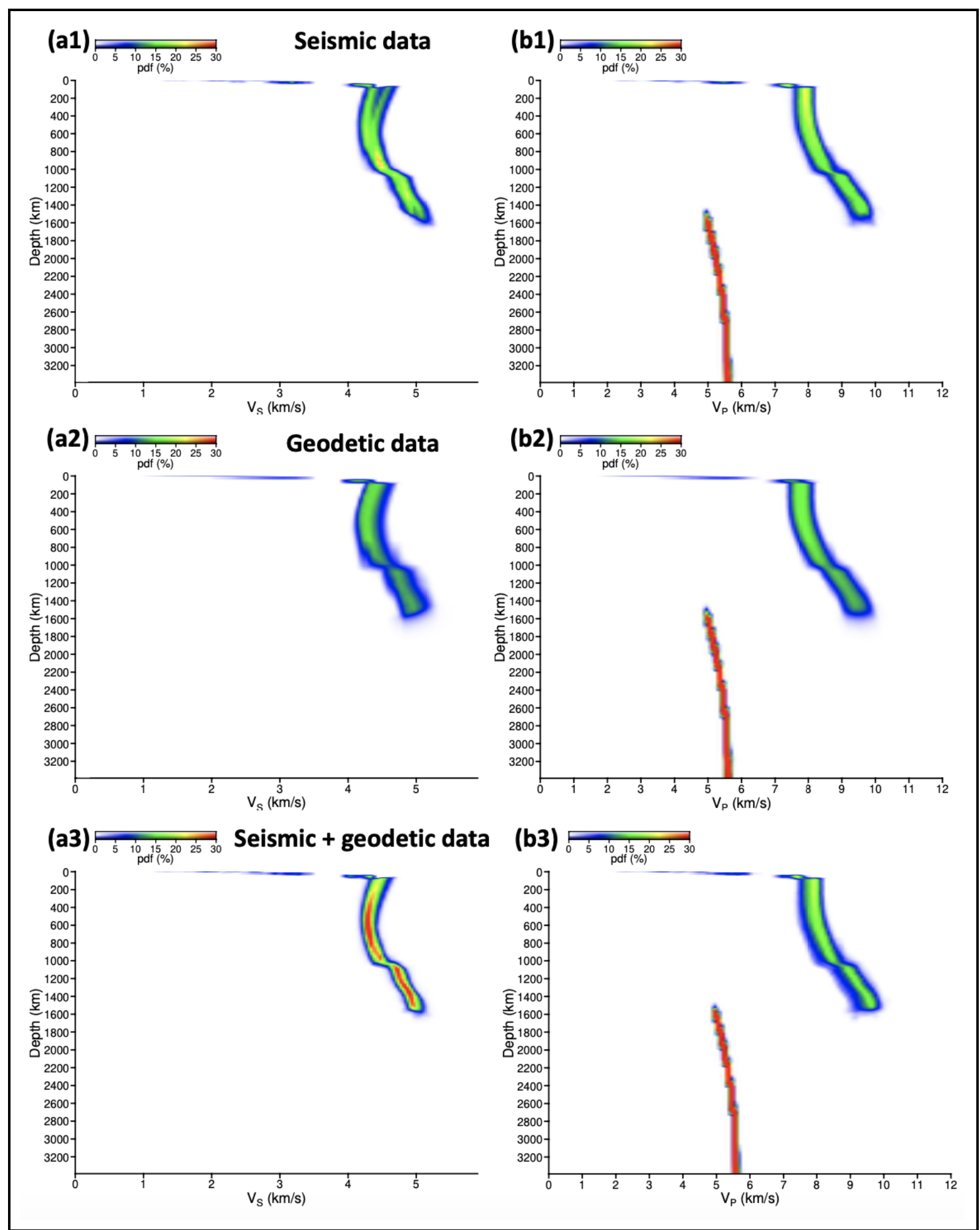

Fig. S9-2. A posteriori probability density functions (pdfs) of the 1-D mantle profiles. The panels are: (a1-a3) $V_{S}$ and (b1-b3) $V_{P}$, considering seismic data alone (top), geodetic data alone (middle), and both seismic and geodetic data (bottom). Red and blue colors refer to small and high probabilities, respectively. The pdf is computed by counting the number of sampled 
profiles in each of the cases. The spacing is $1 \mathrm{~km}$ for depth, and $0.05 \mathrm{~km} / \mathrm{s}$ and 0.1 for $\mathrm{V}_{\mathrm{S}}$ and $\mathrm{V}_{\mathrm{P}}$, respectively. At each given depth, the sum of the pdf over all the velocity intervals equals 100 percent.
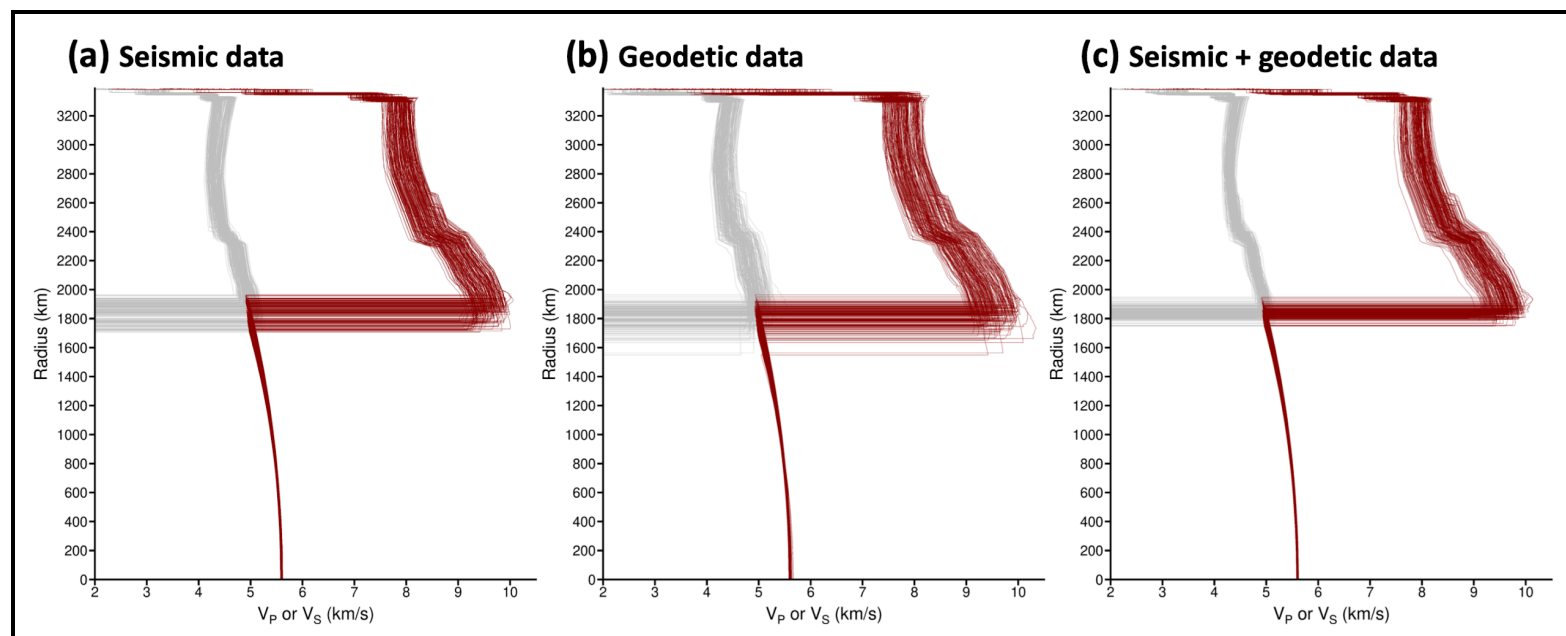

Fig. S9-3. Posterior distribution of models in the geodynamic inversion.

Subsets of 200 random $V_{S}$ (in gray) and $V_{P}$ (in dark red) models as a function of Mars' radius selected from the ensemble solution, considering three different input data: (a) seismic data alone, (b) geodetic data alone, and (c) both seismic and geodetic data. 


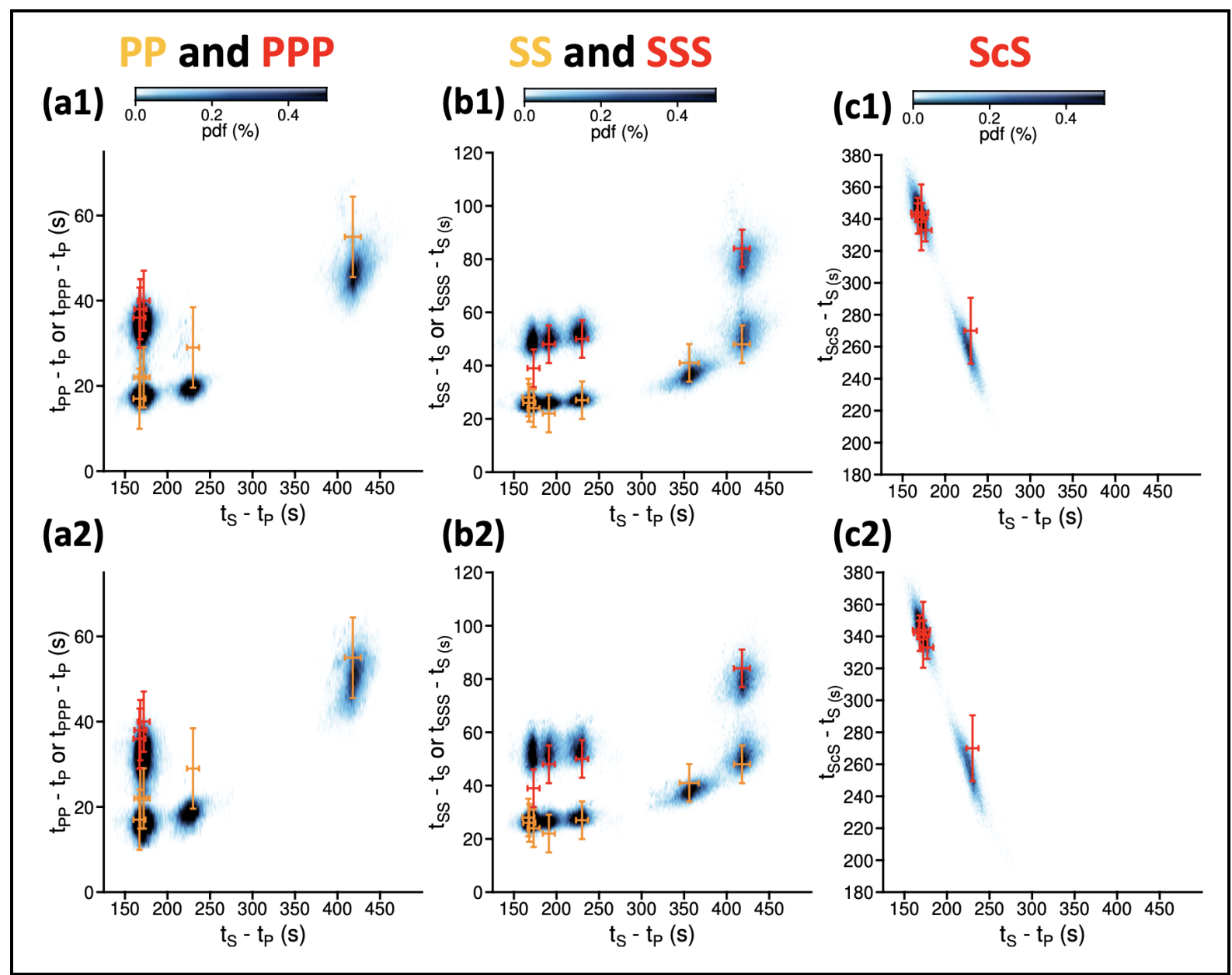

Fig. S9-4. Fit of the seismic data in the geodynamic inversion.

Relative travel times for the "geodynamic" inversion considering seismic data only (a1, b1, c1), and for the inversion considering both seismic and geodetic data (a2, b2, c2). The color scale shows the pdf of the differential arrival times $t_{P P}-t_{P}$ and $t_{P P P}-t_{P}(a 1$ and $a 2), t_{S S}-t_{S}$ and $t_{S S S}-t_{S}(b 1$ and $\mathrm{b} 2$ ), and $\mathrm{tscS}_{\mathrm{Sc}}-\mathrm{t}_{\mathrm{S}}\left(\mathrm{c} 1\right.$ and $\mathrm{c} 2$ ) as a function of $\mathrm{t}_{\mathrm{S}}-\mathrm{t}_{\mathrm{P}}$. The orange and red crosses correspond to the observed data uncertainties. 


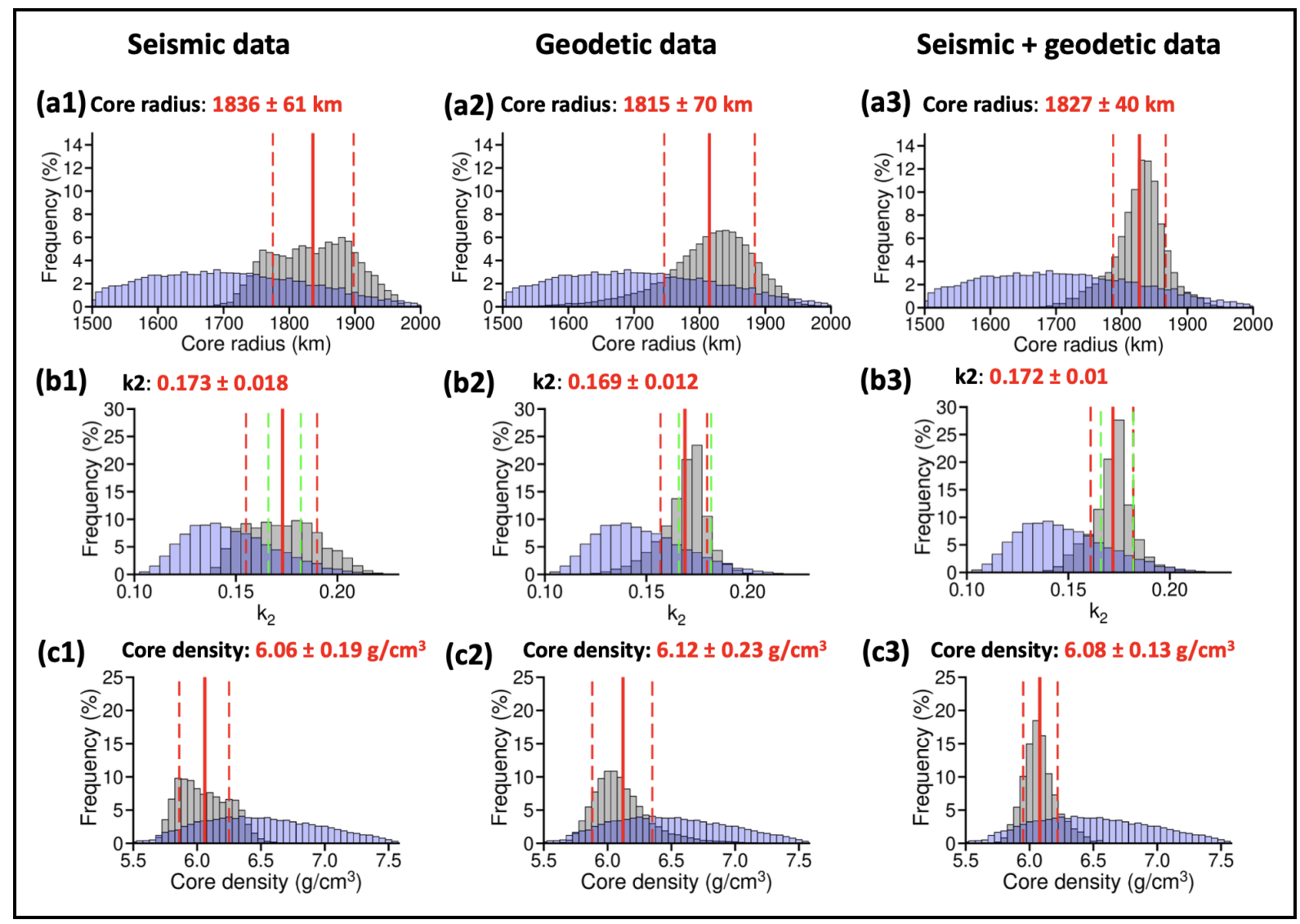

Fig. S9-5. Marginal probabilities for the geodynamic inversion.

Subplots are core radius (a1-a3), $k_{2}$ (b1-b3), and core density (c1-c3). Shown for the inversions using seismic data (left), geodetic data (middle), and both seismic and geodetic data (right). The a priori and a posteriori probabilities are shown in blue and gray, respectively. The mean values and \pm standard deviation are displayed in red. The dashed green lines in (b1-b3) represent the uncertainty bounds on the $k_{2}$ value from (11). 


\section{Differential travel time data fit}

We have calculated predicted differential travel times for the seismic models generated using the geodynamical parameterization. Fig. S10-1 summarizes the differential travel time data fit for all events and picks, sorted by P-S delay time, which is a proxy for epicentral distance. Equivalent data fits were obtained for the seismic and geophysical parameterizations. The differential travel time picks for the surface reflections (PP, PPP, SS, and SSS) are from Khan et al. (2021).

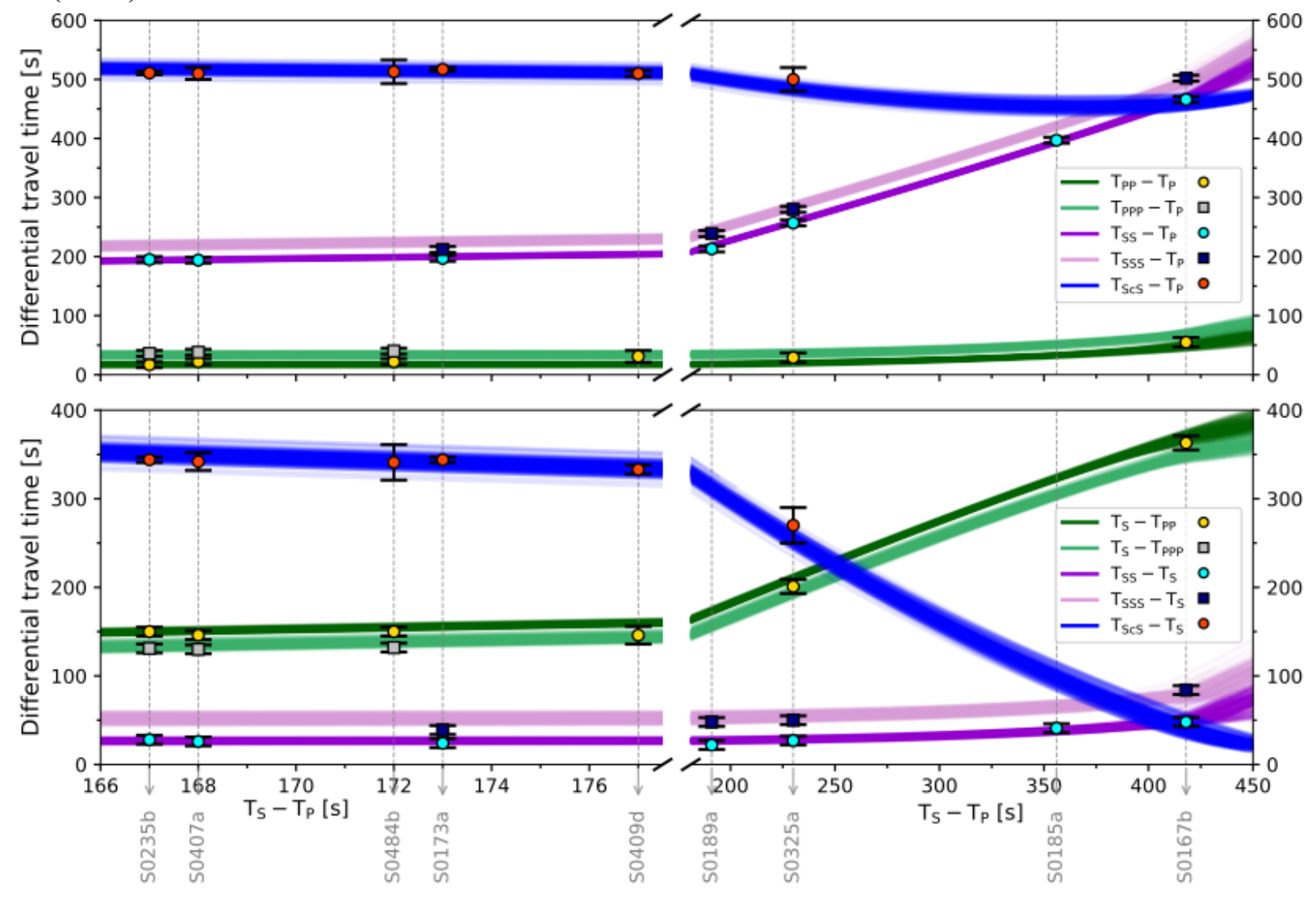

\section{Fig. S10-1. Differential body wave travel time misfits.}

Green and purple lines denote differential travel times computed using the inverted models that are based on the geodynamic parameterization. The upper panel shows times relative to the Pwave arrival and the lower panel shows times relative to the $\mathrm{S}$-wave arrival. Squares and circles indicate the observations together with their uncertainties. 


\section{Core composition}

Various light elements have been suggested to be alloyed to iron in Mars' core. Thermoelastic properties of liquid Fe alloys can be assessed based on thermodynamic solution models that are built starting from those of the end-members and validated against experimental results.

In order to map the average core density to core composition, we solve the differential equations describing the pressure, temperature, and gravity in the core (e.g., (4)) by using the average density as well as the pressure and temperature at the core mantle boundary as boundary conditions for a subset of interior structure models representative of the presently inferred core radius. S is commonly considered to be the main light element alloyed to iron in Mars' core (e.g., (14)). Si is often looked at as major light element in the Earth's core, but the more oxidizing conditions proper to the formation of Mars allow only for a negligible amount of $\mathrm{Si}$ in its core (at trace level e.g., (52)). The solubility of C in S-rich Fe-alloys is low and is estimated to be at the $0.5 \mathrm{wt} \%$-level in a Martian core containing about $15 \mathrm{wt} \% \mathrm{~S}(121)$ with little effect on core density. In contrast, O dissolves readily in S-rich Fe-alloys (122). Finally, H, which could potentially enter the core through hydrated mantle minerals (e.g., $(123,124)$ ), if present at the $\mathrm{wt} \%$ level, is expected to significantly decrease the density of the alloy (65). Thus, as potential light elements here, we consider primarily $\mathrm{S}, \mathrm{O}, \mathrm{C}$, and $\mathrm{H}$. We model the equation of state of the core by assuming that the liquid alloy can be described as an ideal solution with liquid $\mathrm{Fe}(66)$, liquid $\mathrm{FeS}(67,68)$, liquid $\mathrm{FeO}(66), \mathrm{FeH}(65)$, and $\mathrm{Fe}_{3} \mathrm{C}(125)$ as end-members. To account for the density difference between liquid and solid $\mathrm{FeH}$ we decrease the density of $\mathrm{FeH}$ by $2 \%$ and we neglect the effect of $\mathrm{Ni}$ on the elastic properties of the core. To account for the current uncertainties on thermo-elastic properties we here consider for the liquid Fe-S alloys the equation of state from $(67,68)$ for the liquid $\mathrm{Fe}-\mathrm{S}$ alloys. Fe-S models based on the latter require up to $5 \mathrm{wt} \%$ more $\mathrm{S}$ for a given core density.

Modelled core density as a function of S content for a number of different alloys are shown in Fig. S11-1. For an Fe-S core the amount of S required to match the inferred core density is significantly larger than what is deduced from geo- and cosmochemical models $(<13-19$ wt. $\%$, e.g., $(52,55)$, gray horizontal bar) and above the sulfur content Mars would have if it were made of the sulfur-richest meteorites (EH chondrites). Even adding a maximum amount of $5 \mathrm{wt} \%$ of oxygen to the liquid Fe-S alloy would not bring the amount of sulfur in the core in line with formation models. A further significant reduction can be achieved by adding a small fraction of hydrogen and/or carbon to the Fe-O-S alloy. The addition of $1 \mathrm{wt} \%$ of $\mathrm{H}$ together with $1 \mathrm{wt} \%$ of $\mathrm{C}$ reduces the amount of $\mathrm{S}$ to within acceptable bounds for a core density above $5.9 \mathrm{~g} / \mathrm{cm}^{3}$, but more hydrogen would be required $(<1 \mathrm{wt} \%)$ to match the lowest densities found in this study. While the numbers for $\mathrm{O}$ and $\mathrm{H}$, and $\mathrm{C}$ are probably on the high side, they serve to emphasize the need for supplementary light elements in Mars' core to align its mean density derived here with the cosmochemically imposed limits on S. 


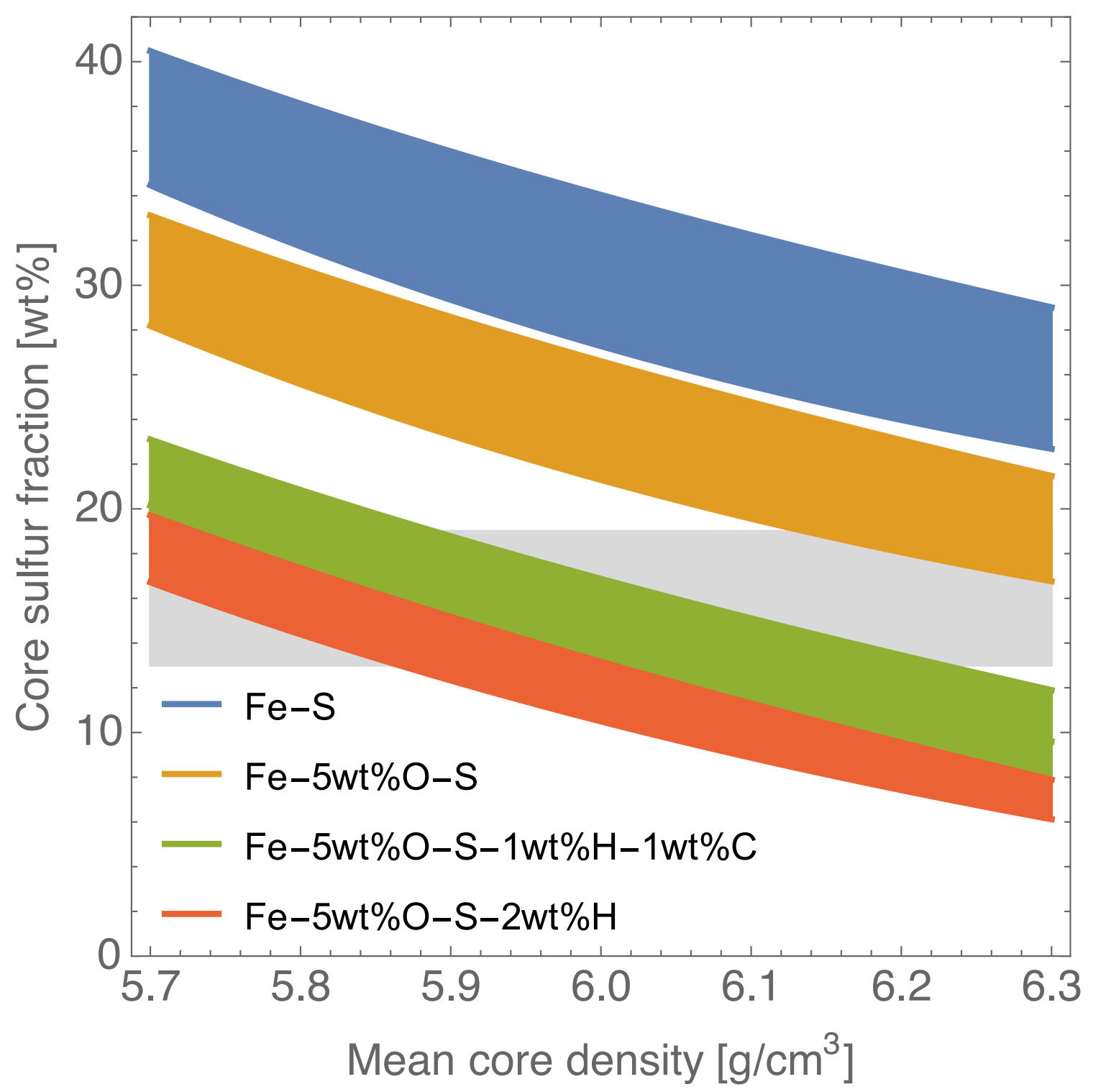

Fig. S11-1. Mean core density as a function of core sulfur fraction.

The plot shows modelled core density as a function of $\mathrm{S}$ content for a number of different alloys. For the calculations performed here, mean core radius $(\sim 1830 \mathrm{~km})$ and density $\left(5.7-6.3 \mathrm{~g} / \mathrm{cm}^{3}\right)$, including core-mantle-boundary pressure $(\sim 19 \mathrm{GPa})$ and temperature $(\sim 1950 \mathrm{~K})$, are equivalent to the models of this study. The thickness of the color bands results from using two different equations of state for liquid Fe-S to model the thermoelastic properties of the core alloy. The lower limit is based on (67), whereas the upper limit relies on (68). The horizontal gray bar represents the amount of $\mathrm{S}$ deduced from cosmochemical models 